\title{
The role of pharmacogenetics in Osteosarcoma
}

Melanie Hagleitner 
The role of pharmacogenetics in Osteosarcoma

\section{PROEFSCHRIFT}

ter verkrijging van de graad van doctor

aan de Radboud Universiteit Nijmegen

op gezag van de rector magnificus prof. dr. Th.L.M. Engelen,

volgens besluit van het college van decanen

in het openbaar te verdedigen op dinsdag 17 maart 2015

om 16.30 uur precies

door

Melanie Margaretha Hagleitner

geboren op 12 oktober 1978

te Zell am See (Oostenrijk)

ISBN 978-90-9028836-9

Coverdesign and book layout:

Johanna Meinschad, www.marketingdienstleistung.at

Printing: Ipskamp, Enschede

The printing of this thesis was financially supported by Radboud umc

Copyright: No part of this book may be reproduced, stored in a retrieval system or transmitted in any form or by any means, without written permission of the author or, when appropriate, of the scientific journal in which parts of this book have been published. 
Promotoren:

Copromotoren:

Manuscriptcommissie:

Prof. dr. H.N. Caron (UvA)

Prof. dr. F.G.M. Russel

Prof. dr. P.M. Hoogerbrugge

Prof. dr. H.J. Guchelaar (LUMC)

Dr. D.M.W.M. te Loo

Dr. M.J.H. Coenen

Meinen Brüdern Michael und Martin 



\title{
CONTENTS
}

\author{
Chapter 1 Introduction \\ page 9 \\ Chapter 2 \\ page 19 \\ Age as prognostic factor in patients with osteosarcoma \\ Bone. 2011;49:1173-1177
}

Chapter 3

Survival trends and long term toxicity in pediatric

page 35

patients with osteosarcoma

Sarcoma. 2012;636405

Chapter 4

page 47

Taqman genotyping assays can be used on decalcified and paraffin embedded tissue from patients with osteosarcoma Pediatr Blood Cancer. 2011;56:35-38

Chapter 5 page 57

Chapter 6 page 75

Chapter 6B page 89

Chapter 7 page 95

Chapter 8 page 111

Chapter 9 page 125
A pilot study: Genetic variants may influence methotrexate plasma concentrations in pediatric malignancies

The role of the MTHFR 677C>T polymorphism in methotrexate -induced liver toxicity: a meta-analysis in patients with cancer Pharmacogenomics J. 2014;14:115-119

Is there a role for the MTHFR $677 \mathrm{C}>\mathrm{T}$ and the $1298 \mathrm{~A}>\mathrm{C}$ polymorphism methotrexate induced liver toxicity? Pharmacogenomics. 2014;15:1401-1403

Minor influence of genetic variants in the TPMT and COMT genes regarding cisplatin induced hearing loss in patients with cancer: a meta-analysis including two new cohorts PLoS One. 2014;9:e115869

A first step towards personalized medicine in osteosarcoma: Pharmacogenetics as predictive marker of outcome after chemotherapy based treatment submitted

General discussion 


\section{CHAPTER 1}

Samenvatting

\section{Introduction}

Dankwoord

page 146

About the author

page 149

List of publications

page 150

List of abbreviations

List of a 


\section{Osteosarcoma}

Osteosarcoma is the most common primary tumor of the bone, which may occur at al ages but is seen predominantly in adolescents. Nevertheless, it is still a rare disease with a worldwide incidence rate of 3-4 cases per million per year ${ }^{1}$. Untreated, osteosarcoma is fatal. Aggressive local growth and the tendency to metastasize mark its course. In the 1970s when surgery was the only treatment option, the prognosis for patients with osteosarcoma was poor, with long-term survival rates of less than $20 \%{ }^{2}$. Today, therapy is changed to a multi-disciplinary approach, focusing on both local and systemic manifestations of osteosarcoma, incorporating surgery and chemotherapy. The use of this multi-disciplinary approach has resulted in survival rates of $60-65 \%$ since the late $1980 \mathrm{~s}^{3}$. Whereas in other forms of cancer clear progress has been made in the previous decades, either by the use of new drugs or by the ability of risk stratification and subsequently treatment adjustments ${ }^{4}$ in patients with osteosarcoma no substantial improvement in disease free survival (DFS) has been achieved.

The specific roles of various chemotherapeutic agents in the treatment of osteosarcoma have been the subject of many studies in the last three decades. There is considerable agreement among specialists about the main components: cisplatin and doxorubicin which are the most active drugs and are virtually part of every treatment protocol since the 1980s. Several studies have reported superior results using regimens containing these two drugs in addition to other agents such as methotrexate (MTX) and ifosfamide ${ }^{5-7}$. In the recent study trial of the European and American Osteosarcoma Study Group (EURAMOS) the combination of cisplatin, doxorubicin and high-dose methotrexate forms the standard treatment arm. The addition of ifosfamide with or without etoposide remains controversia| $\left.\right|^{8-10}$ and is now being studied in a randomized setting in EURAMOS I.

The ratio to introduce pre-operative chemotherapy in the late 1970 s was based on the development of limb-sparing procedures. Pre-operative chemotherapy was used to bridge the gap between biopsy and resection as the manufacturing of custom made limb-sparing endoprotheses took several weeks ${ }^{11}$. With this approach, it is now possible to evaluate the efficacy of the treatment by assessing the response of the tumor to pre-operative therapy ${ }^{12}$. Numerous studies have shown a strong correlation between tumor necrosis greater than $90 \%$ and a better prognosis ${ }^{12-16}$. This histological response is currently the most sensitive indicator of survival. The contribution of traditional prognostic factors like age, gender, tumor site and size were subject of numerous studies but results are contradictive and therefore there is no consensus whether these prognostic variables might be used to stratify patients before the onset of therapy ${ }^{17}$. The prognostic value of histological response led to the hypothesis that intensification of chemotherapy may increase survival in poor responders. However, intensification of preoperative treatment did not improve long-term outcomes ${ }^{18}$. Moreover, when preoperative therapy was lengthened, the histological response loses its prognostic value ${ }^{18}$.
The absence of reliable predicting factors for risk stratification resulted in similar treatment schedules for all osteosarcoma patients. In fact, there are no adaptations in post-operative therapy in patients with poor response to the pre-operative treatment. As such, it appears that we have reached the limit in the survival of osteosarcoma patients achievable with currently available chemotherapy. Several new chemotherapeutic drugs have been investigated over the past three decades. However, only few agents have shown clinical benefit. The use of monoclonal antibodies such as trastuzumab, which targets the epiderma growth factor receptor 2 , has been studied in a phase II trial showing no improvement in outcome ${ }^{19}$. The effect of interferon alpha (IFN a) is currently studied in the EURAMOS trial. Preliminary data show a 3 -year DFS of $77 \%$ in patients who were randomized to have interferon injections compared to $74 \%$ of those who were randomized to have chemotherapy alone ${ }^{20}$. Treatment with bisphosphonates such as pamidronate or zoledronic acid in addition to chemotherapy is only applied to a hand full of patients and the clinical benefit of these agents need to be studied in a prospective randomized trial ${ }^{21,22}$. As the incidence of osteosarcoma is between 3 and 4.5 cases per million, it will take several years to include a large number of patients in a prospective trial to study the efficacy of these new agents. Furthermore, the a priori knowledge of which patients will benefit from new agents will help to define new strategies in order to improve outcome and decrease the burden of late effects. We hypothesized that pharmacogenetics may help to stratify patient into good responders and bad responders. More insight in the genetic determinants involved in drug response may help in designing more effective therapeutic approaches, e.g. targeted cancer therapy for patients not responding on standard therapy (Figure I) 
Figure I. Application of pharmacogenetics to stratify the individual patient

Patients with same diagnosis

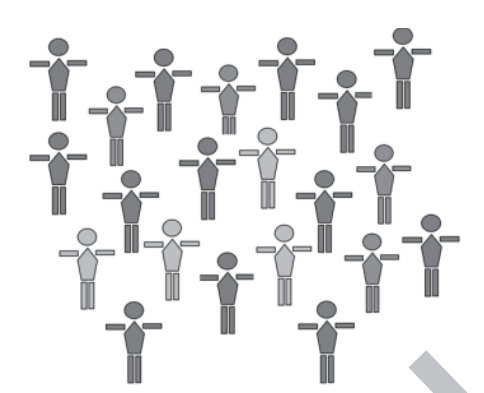

Alternative treatment

Non-responders Toxic-responders
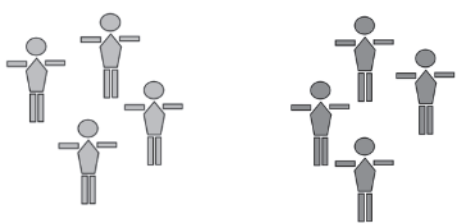

Standard treatment

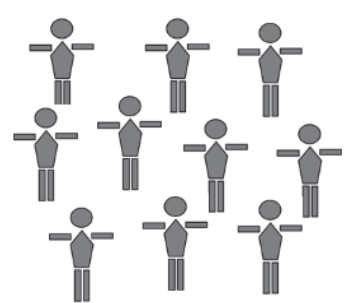

\section{Pharmacogenetics}

Using pharmacogenetics, we investigated inherited genetic variations which might affect individual responses to drugs. Many different methods are used to investigate the role of genetic variants in genes coding for proteins involved in the metabolism of drugs used for a certain disease. These include candidate gene approaches, pathway-gene approach and genome wide association studies (GWAS). Each of these techniques is useful in a certain way but has also limitations (Figure II).

Figure II. Different methods to investigate the role of genetic variants in drug related genes.

$$
\begin{aligned}
& \text { Single gene approach Genome wide approach } \\
& \begin{array}{ll}
\text { - Analyses favourite candidate gene } & \text { Requires a large cohort to test the } \\
\text { association } & \text { Requires larger cohorts to test } \\
\text { association }
\end{array} \\
& \text { association } \\
& \text { Carries the risk of not finding an } \\
& \text { association }
\end{aligned}
$$

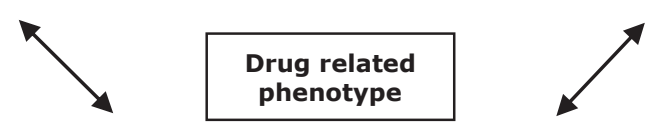

Candidate pathway gene approach

- Analyses several functionally related Analyses several
candidate genes

Requires a smaller cohort to test the association

- Carries the risk of missing important

genes

Provides a more biologically
meaningful association

The candidate gene approach is a hypothesis driven method that investigates genetic variants in genes known to be associated with the trait of interest e.g. metabolism of the used medication. However, replication studies often show inconsistent results making it difficult to interpret the clinical value of these studies ${ }^{23-28}$, e.g. these problems may be addressed by combining results from different studies in a meta-analysis. GWAS on the other hand is a hypothesis free approach, testing thousands/millions of genetic variants in one test that is aimed to discover genes not previously known to be associated with the trait. It is useful method to determine the most significant single nucleotide polymorphisms (SNPs) associated with a certain phenotype in a high-density set of SNPs. A disadvantage of the GWAS however, is the discovery of false positive associations and the often weak statistical signals. When using the GWAS technique, one needs large patient cohorts for discovery, and validation. As the incidence of osteosarcoma patients is low, such large cohorts are not available. For this reason, we performed a pharmacogenetic pathway approach in- 
cluding the pathways of the three main chemotherapeutic drugs used in the treatment of osteosarcoma patients in the past three decades; pharmacogenetic studies have already been described in adults with cancer leading even to adjustment of therapy in patients with breast cancer ${ }^{29-31}$. In childhood cancer, studies have been performed investigating the presence of SNPs of one candidate gene involved in the metabolism of one drug. One of the first results of these pharmacogenetic studies was the identification of a polymorphism in the gene thiopurine-S-methyltransferase (TPMT) that were related to therapy outcome in children with acute lymphoblastic leukemia (ALL) ${ }^{32}$.

As doxorubicin and cisplatin are the cornerstones of the treatment of osteosarcoma patients, we primarily focused on genes and genetic variants involved in the metabolism of these two drugs. The third drug often used in the treatment of osteosarcoma is MTX. Although results are not conclusive, it is suggested that at least in pediatric patients with osteosarcoma MTX plays an important role ${ }^{33}$. For this reason MTX is included in many protocols and the influence of pharmacogenetic factors in the pharmacological effect of MTX (pharmacokinetics and dynamics) is also subject of this thesis.

\section{Objectives and outline of this thesis}

The main objective of this thesis was to define a patient's genetic profile predicting the response to therapy, with the ultimate goal to develop individualized, improved, treatment schedules for patients with osteosarcoma based on their genetic profiles. In addition, we tried to identify patients with an increased risk of developing treatment-related toxicity, e.g. ototoxicity and hepatotoxicity. In future studies these profiles might be used prospectively, leading to adjustment of therapy without jeopardizing outcome.

In chapter $\mathbf{2}$ we describe a retrospective analysis of pediatric patients with osteosarcoma and compared this to adult patients to study whether age has an influence on outcome. I has been described that there are expression changes in enzymes during aging. E.g., the enzymes of the p450 cytochromes (CYPs) system change markedly during the fetal period to the first year of life ${ }^{34}$. However, limited data are available on the expression changes effect between the age of 10 and 40 years, where osteosarcoma is predominantly diagnosed. A recently published study describes age related variation in drug transporter expression showing significantly lower expressions levels in all pediatric age groups compared to adults ${ }^{35}$. In addition, other pharmacological processes like absorption and excretion of drugs might differ during growth and development. This might influence the response to the chemotherapeutics given to patients in both efficacy and toxicity.

The rareness of the disease forced us to include patients over a lengthy period of 30 years. Hence, in chapter $\mathbf{3}$ we describe survival trends and analysed the occurrence of long term toxicity over the last three decades to gain more insight what could have influenced outcome in our patient group.
With a 5 -year overall survival of approximately $50-60 \%$, archived histological specimens were often the only accessible source of biological material. Chapter $\mathbf{4}$ describes the process of isolating germ-line DNA from decalcified formalin-fixed, paraffin-embedded (FFPE) tissue. The most active drugs in the treatment of osteosarcoma are cisplatin, doxorubicin and high-dose MTX.

The use of multi-agent therapy led to an enhanced survival of osteosarcoma patients but, this concept is unfortunately also associated with acute and long-term toxicity. In the treatment of high-dose MTX a threshold peak level is needed to achieve good response on chemotherapy. On the other hand, high concentration levels lead to an increased risk of toxicity. In chapter $\mathbf{5}$ we describe the influence of genetic variation in the elimination of MTX. We investigated if SNPs in genes involved in the folate pathway, the key pathway in the processing of MTX, are associated with delayed MTX elimination and/or MTX induced toxicity.

One of the most common side effects of MTX is hepatotoxicity. The most studied SNP with re spect to MTX-induced hepatotoxicity is the $677 \mathrm{C}>\mathrm{T}$ variant in the methylenetetrahydrofolate reductase (MTHFR) gene. However, not all studies have been able to link presence of the $677 T$ allele to an increased risk of MTX-induced toxicity. For this reason, we performed a meta-analysis including 1044 cancer patients which is described in chapter 6.

Hearing loss is one of the most common long-term side effects of cisplatin. Patients with osteosarcoma are more prone to ototoxicity due to the high cumulative dose of cisplatin during treatment. Recently, a candidate gene study suggested that genetic variants in the genes encoding thiopurine S-methyltransferase (TPMT) and catechol O-methyltransferase (COMT) can predict the development of cisplatin-induced ototoxicity ${ }^{36}$. A recently published study ${ }^{37}$ however, failed to validate these findings and showed inconsistent results. The aim of the study described in chapter 7 was to provide a clearer picture of the genetic impact of TPMT and COMT variants on developing cisplatin-induced ototoxicity by performing the largest meta-analysis to date including 664 patients with cancer.

Treatment of patients with osteosarcoma seems to have substantial shortcomings in optimal strategies compared to other types of cancer. The most consistent prognostic facto for outcome is the presence of metastatic lesions at time of diagnosis. However, treatmen management of this patient group follows the same principles as the treatment of patients with localized disease. Several studies have reported that histologic response after neoadjuvant therapy is of prognostic value for survival, but it cannot be assessed at time of diagnosis. Further advances in treatment will however be dependent on reliable predicting factors for risk stratification. In chapter $\mathbf{8}$ we report the influence of genetic variants on outcome. We hypothesized that by identifying the genetic variants in genes relevant for the response of chemotherapeutic treatment it may be possible to stratify treatment of patients immediately after diagnosis and offer the possibility to improve treatment and subsequently progression free survival of patients with osteosarcoma.

A general discussion of the studies that are presented in this thesis is given in chapter $\mathbf{9}$. 


\section{References}

Mirabello L, Troisi RJ, Savage SA. Osteosarcoma incidence and survival rates from 1973 to 2004: data from Results Program. Cancer. 2009;115:1531.

2 Bacci $G$, Picci P, Ferrari S, et al. Primary chemotherapy and delayed surgery for nonmetastatic osteosarcom of the extremities: Results in 164 patients preoperatively
by cisplatin and doxorubin. Cancer. 1993; $72: 3227-3230$. 3 Link MP, Gebhardt MC, Meyers PA. Osteosarcoma. In: Pizzo PA, Poplack DG, editors. Principles and practice
of pediatric oncology. 2nd ed. Philadelphia: Lippincott Williams \& Wilkins. 2002; p.1502-1589.

4 Pui CH, Mullighan CG, Evans WE, et al. Pediatric acute lymphoblastic leukemia: where are we going and how
do we get there? Blood. 2012;120:1165-1174.

Bacci $\mathrm{G}$, Ferrari $\mathrm{S}$, Delepine $\mathrm{N}$, et al. Predictive factors of histologic response to primary chemotherapy in osteosarcoma of the extremity: study of 272 patients preoperatively treated with high-dose methotrexat
doxorubicin, and cisplatin. 1 Clin Oncol. 1998:16:658-663.

Fuchs $\mathrm{N}$, Bielack SS, Epler D, et al. Long-term results of the co-operative German-Austrian-Swiss osteosarthe limbs. Ann Oncol. 1998;9:893-899.

7 Meyers $\mathrm{PA}$, Gorlick R, Heller $\mathrm{G}$, et al. Intensification of preoperative chemotherapy for osteogenic sarcoma:
results of the Memorial Sloan-Kettering (T12) protocol. 3 Clin Oncol. 1998;16:2452-2458.

Goorin AM, Harris MB, Bernstein M, et al. Phase II/III trial of etoposide and high-dose ifosfamide in newly
diagnosed metastatic osteosarcoma: a pediatric oncology group trial. J Clin Oncol. 2002;20:426-433.

Harris MB, Gieser P, Goorin AM, et al. Treatment of metastatic osteosarcoma at diagnosis: a Pediatric Oncolo-
gy Group Study. J Clin Oncol. 1998; $16: 3641-3648$.

Meyers $P$, Schwartz $C$, Bernstein $M$, et al. Addition of ifosfamide and muramyl tripeptide to cisplatin, doxoru-
bicin, and high-dose methotrexate improves event free survival (EFS) in localized osteosarcoma (OS) [ab-

11 Federman N, Bernthal $\mathrm{N}$, Eilber $\mathrm{FC}$, et al. The multidisciplinary management of osteosarcoma. Curr Treat
Options Oncol. 2009;10:82-93.

12 Rosen $G$, Marcove RC, Caparros $B$, et al. Primary osteogenic sarcoma: the rationale for preoperative chemo-

Winkler $K$, Beron $G$, Delling $G$, et al. Neoadjuvant chemotherapy of osteosarcoma: results of a randomized
cooperative trial (COSS-82) with salvage chemotherapy based on histological tumor response. J Clin Oncol. 1988;6:329-337.

14 Hudson $M$, Jaffe $M R$, Jaffe $N$, et al. Pediatric osteosarcoma: therapeutic strategies, results, and prognostic

15 Goorin AM, Schwartzentruber DJ, Devidas M, et al. Presurgical chemotherapy compared with immediate surgery and adjuvant chemotherapy for nonmetastatic osteosarcoma: Pediatric Oncology Group Study POG-
8651 . I Clin Oncol. 2003;21:1574-1580.

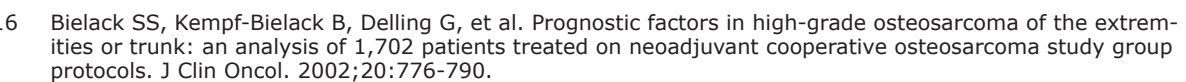

17 Davis AM, Bell RS, Goodwin PJ. Prognostic factors in osteosarcoma: a critical review. J Clin Oncol. 1994;423-

18 Lewis IJ, Nooij MA, whelan J, et al. Improvement in histologic response but not survival in osteosarcoma patients treated with intensified chemotherapy: a
Intergroup. I Natl Cancer Inst. 2007;99:112-128.

Ebb D, Meyers $\mathrm{P}$, Grier $\mathrm{H}$, et al. Phase II trial of trastuzumab in combination with cytotoxic chemotherapy
for treatment of metastatic osteosarcoma with human epidermal growth factor receptor 2 overexpression: for treatment of metastatic osteosarcoma with human epidermal growth factor receptor 2 overexpression: a
report from the children's oncology group. 3 Clin Oncol. 2012;30:2545-2551.

20 Bielack SS, Smeland S, whelan J, et al. MAP plus maintenance pegylated interferon \{alpha\}-2b (MAP-IFN) EUAMOS-1 good response randomization. ASCO MEETING preoperative MAP: :irst results
ABSTRACTS 2013:LBA10504.

21 Meyers $\mathrm{PA}$, Healey $\mathrm{JH}$, Chou AJ, et al. Addition of pamidronate to chemotherapy for the treatment of osteo-
sarcoma. Cancer. 2011;117:1736-1744.

22 Goldsby $\mathrm{RE}$, Fan $\mathrm{TM}$, Villaluna $\mathrm{D}$, et al. Feasibility and dose discovery analysis of zoledronic acid with concurrent chemotherapy in the treatment of newly diagnosed metastatic osteosarcoma: a report from the Chil-
dren's Oncology Group. Eur $J$ Cancer. 2013;49:2384-2391.

23 Fisher MC, Cronstein BN. Metaanalysis of methylenetetrahydrofolate reductase (MTHFR) polymorphisms
affecting methotrexate toxicity. J Rheumatol. 2009;36:539-545.
24 Lee YH, Song GG. Associations between the C677T and A1298C polymorphisms of MTHFR and the efficacy

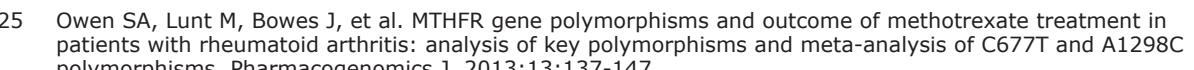

26 Yang $L, H u X, X u$. Impact of methylenetetrahydrofolate reductase (MTHFR) polymorphisms on methotrex-
ate-induced toxicities in acute lymphoblastic leukemia: a meta-analysis. Tumor Biol. 2012; 33:1445-1454. 27 Lopez-Lopez E, Martin-Guerrero I, Ballesteros J, et al. A systematic review and meta-analysis of MTHFR poly-
morphisms in methotrexate toxicity prediction in pediatric acute lymphoblastic leukemia. Pharmacogenomics
J. 2013;13:498-506.

28 Hagleitner MM, Coenen MJ, Aplenc R, et al. The role of the MTHFR 677C>T polymorphism in methotrex-

29 Deeken JF, Figg WD, Bates SE, et al. Toward individualized treatment: prediction of anticancer drug disposi-

With pharmacogenetics. Anticancer Drugs. 2007:18:111-126.

30 Blackhall $\mathrm{FH}$, Howell S, Newman B. Pharmacogenetics in the management of breast cancer-prospects for

31 Aplenc $\mathrm{R}$, Lange B. Pharmacogenetic determinants of outcome in acute lymphoblastic leukaemia. $\mathrm{Br} \mathrm{J}$ Hae-
matol. 2004;125:421-434.

32 Ross CJ, Katzov-Eckert H, Dubé MP, et al. Genetic variants in TPMT and COMT are associated with hearing
loss in children receiving cisplatin chemotherapy. Nat Genet. 2009;41:1345-1349.

33 van Dalen EC, van As JW, de Camargo B. Methotrexate for high-grade osteosarcoma in children and young
adults. Cochrane Database Syst Rev. 2011;5.

34 Kearns GL, Abdel-Rahman SM, Alander SW, et al. Developmental pharmacology - drug disposition, action,
and therapy in infants and children. N Engl J Med. 2003;349:1157-1167.

Intestinal Transporter Gene Expression during Childhood: Age Matters. Drug Metab Dispos. 2014;42:1268-

Ross CJ, Katzov-Eckert H, Dubé MP, et al. Genetic variants in TPMT and COMT are
loss in children receiving cisplatin chemotherapy. Nat Genet. 2009;41:1345-1349.

37 Yang JJ, Lim JY, Huang J, et al. The role of inherited TPMT and COMT genetic variation in cisplatin-induced 



\section{CHAPTER 2}

\section{Age as prognostic factor in patients with osteosarcoma}

Melanie M. Hagleitner

Peter M. Hoogerbrugge

Winette T.A. van der Graaf

Uta Flucke

H.W. Bart Schreuder

D. Maroeska W.M. te Loo 


\section{Abstract}

Age at diagnosis is a well-known prognostic factor in many different malignancies; its significance for patients with osteosarcoma is however controversial. To gain more insight in the prognostic role of age, we performed a retrospective study at our institute. We included 102 patients with de-novo osteosarcoma and formed three age groups to evaluate age specific survival rates: $\leq 14$ years, $15-19$ years and $20-40$ years. Differences in outcome between patients aged 15-19 years treated at either the pediatric department or the adult department of oncology were evaluated. The 5 -year overall survival rate (OS) of the whole population was $53.5 \% \pm 1.5 \%$. OS of $70.6 \% \pm 0.8 \%$ was seen in patients $\leq 14$ years old, $52.5 \% \pm 1.1 \%$ in patients $15-19$ years old and $33.3 \% \pm 0.9 \%$ in the patients aged $20-40$ years $(p=0.01)$. Significant differences were observed with regard to stage at presentation (higher in older age groups), size of the tumor (larger in younger age groups) and histological response (more good responders in younger age groups). No significant difference was seen between outcomes of patients aged 15-19 years treated at the pediatric or adult oncology department. In conclusion, younger patients have a significantly better outcome than older patients.

\section{Introduction}

Osteosarcoma is the most common primary tumor of the bone, which may occur at all ages but is seen predominantly in adolescents. Nevertheless, it is still a rare disease with a worldwide incidence rate of 3-4 cases per million per year ${ }^{1}$. Until the seventies, osteosarcoma had a very poor prognosis. Major progress was made with the introduction of adjuvant chemotherapy. Disease-free survival (DFS) rates increased from below $20 \%$ with surgery only to more than $40 \%$ with adjuvant chemotherapy ${ }^{2-4}$. Unfortunately, osteosarcoma is relatively resistant to many chemotherapeutics leading to a rather short list of effective drugs. The majority of the currently used treatment protocols are based on a combination of doxorubicin, cisplatin, methotrexate (MTX), and/or ifosfamide. Introduction of neoadjuvant and adjuvant chemotherapy in the context of more intensive multiagent regimens has led to a survival of $60 \%^{5-8}$. However, it seems that survival rates have reached a plateau. Treatment related toxicity and mortality are the main limiting factors allowing no further treatment intensification. E.g., higher dosage of chemotherapy resulted in more long-term complications like cardiac failure ${ }^{9,10}$.

Identification of patients with a favorable prognosis and those with a poor prognosis may be used to adjust treatment schedules. Generally accepted prognostic factors for survival of osteosarcoma are presence of metastases at time of diagnosis, histologic response to neoadjuvant chemotherapy and adequate surgical margins. The prognostic relevance of other factors such as tumor site and size, histologic subtype, elevated alkaline phosphatase (AP), elevated lactate dehydrogenese (LDH) and age are still controversial. Although age at time of diagnosis is a well-known prognostic factor in many different malignancies ${ }^{11,12}$ its relevance in the treatment of osteosarcoma patients is still not clear. There are several reports in literature about outcome of patients older than 40 years versus younger than 40 years showing that age above 40 years is associated with a worse outcome ${ }^{13,14}$. However, highest incidence of osteosarcoma is seen in the younger patient group with a peak incidence at the adolescence age. There are some reports showing a favorable outcome for osteosarcoma patients below 14 years ${ }^{15-17}$ others report that the outcome is worse for this same age group ${ }^{18,19}$. Because of these contradictive reports, the relevance of age as prognostic factor in patients with osteosarcoma needs further elucidation. For this reason, we retrospectively analyzed the role of age in a cohort of patients up to the age of 40 years, with both, metastatic and non-metastatic osteosarcoma.

\section{Patients and Methods}

\section{Patients}

We treated 112 osteosarcoma patients in our institution between 1979 and 2008 with newly diagnosed, high-grade osteosarcoma. All patients were aged below 40 years. None of the patients was known with Paget's disease. We excluded two patients with a histo- 
ry of exposure to radiation due to prior malignancy and 8 patients with an incomplete dataset. In total, 102 patients were included in this analysis. Median age at diagnose was 17.8 years ranging from 4.5 to 39.5 years. Consistent with previous reports in the literature ${ }^{16,17,20}$ different age groups were defined starting with an age group $\leq 14$ years. Subsequently a second group aged 15-19 years was defined. The third group consisted of patients aged 20-40 years. Recently, studies in adolescents with acute lymphoblastic leukemia (ALL) indicated that patients treated in pediatric departments had a higher probability of survival compared to those treated in adult departments ${ }^{21}$. To evaluate whether outcome in patients with osteosarcoma is related to treatment at a pediatric or adult department, this was studied in the patients with age between 15-19 years treated either at the department of pediatrics or treated at the department of medical oncology.

\section{Treatment}

\section{Chemotherapy}

Treatment was performed according to the protocol used at time of enrollment. From 1979 to 2008, four consecutive protocols were used (Table I): EORTC 20781, EORTC 80831 EORTC 80931, and Euramos.

Table I Cumulative Dose per protocol

\begin{tabular}{|lcccccc|}
\hline & \multicolumn{2}{c}{ Doxorubicin $\mathbf{m g} / \mathbf{m}^{\mathbf{2}}$} & \multicolumn{2}{c|}{ Cisplatin $\mathbf{m g} / \mathbf{m}^{\mathbf{2}}$} & \multicolumn{2}{c|}{ Methotrexate $\mathbf{g} / \mathbf{m}^{\mathbf{2}}$} \\
Protocol & reg A & reg B & reg A & reg B & reg A & reg B \\
EORTC 20781 & 420 & 420 & - & - & 42 & 42 \\
EORTC 80831 & 450 & 300 & 600 & 400 & - & 36 \\
EORTC 80931 & 450 & $450 *$ & 600 & $600 *$ & - & - \\
EURAMOS & 450 & 450 & 480 & 480 & 144 & 144 \\
\hline
\end{tabular}

*G-CSF, granulocyte-colony stimulating factor

\section{Treatment adjustments}

Reduction of chemotherapy was defined as more than $15 \%$ reduction of chemotherapy as compared to the planned cumulative dosage.

\section{Histological response}

In patients treated with neoadjuvant chemotherapy, the histological response of the tumor was determined by evaluating the percentage of tumor necrosis in the removed material. The response to preoperative chemotherapy was defined 'good' if the amount of viable tumor was reports as less than $10 \%$ of the tumor area and 'poor' in the remaining cases. This is according the international criteria as defined by Rosen et $\mathrm{a}^{22}$

\section{Surgery}

The surgical procedure depended on the location and extension of the tumor. Surgical marins were categorized following the criteria defined by Enneking as radical, wide, margina and inadequate if margins were contaminated ${ }^{23}$.

\section{Statistical Analysis}

Overall survival (OS) and disease-free survival (DFS) were estimated using the Kaplan-Meier method ${ }^{24}$. OS was defined as the interval between diagnosis and death from any cause. Patients alive at the date of last follow-up were censored at that time point. DFS was defined as the interval between diagnosis and disease progression/recurrence. Patients without disease recurrence at the date of last follow-up were censored at that date.

Survival and potential prognostic variables were analyzed using cox regression. The clinica characteristics examined included age at diagnosis, gender, site and size of the primary tumor, histological type, presence of metastases, serum alkaline phosphatase (AF) level and serum lactate dehydrogenase (LDH) level. Treatment characteristics examined included chemotherapy, histological response, type of surgery and surgical margins. Factors found to influence prognosis by univariate analysis were analyzed by stepwise multivariate analysis using Cox's proportional hazard regression mode ${ }^{25}$. All analyses were performed using SPSS statistical analysis software (version 16.0, SPSS Inc, Chicago, IL). 


\section{Results}

\section{Patients}

The median follow-up was 5.6 years (range 2.4 to 30 years). The 5 -year OS was $53.5 \%$ $\pm 1.5 \%$ and the 5 -year DFS was $47.1 \% \pm 1.6 \%$. All deaths $(\mathrm{N}=49)$ were related to osteosarcoma with 17 deaths due to progression of the disease, two patients died after local recurrence and 30 after distant metastases (lung: $\mathrm{N}=24$, other: $\mathrm{N}=4$, both: $\mathrm{N}=2$ ).

As mentioned before, patients were divided in three subgroups regarding age. The age groups differed not significantly in the distribution of clinical and treatment related variables as is shown in Table II. Interestingly, patients $\leq 14$ years had less frequently metastases at diagnosis compared to older patients ( $8.8 \%$ vs. $25.9 \%, p=0.07)$, but were at the same time more likely to have tumors $>10 \mathrm{~cm}(55.8 \%$ vs. $26.0 \%, p=0.053)$. Both having metastasis and/or having a tumor of more than $10 \mathrm{~cm}$ is associated with a worse outcome ${ }^{13,26}$. Regarding tumor location in the axial skeleton which is also associated with a poorer outcome, the number of cases arising from these sites in this series is greater in the older group but not statistically different ( $5.9 \%$ vs. $14.8 \%, p=0.24$, Table II and III)

Table II. Patient characteristics according to age

\begin{tabular}{|c|c|c|c|}
\hline Variables & $\begin{array}{c}\leq 14 \text { years } \\
(N=34)\end{array}$ & $\begin{array}{c}15-19 \text { years } \\
(\mathrm{N}=41)\end{array}$ & $\begin{array}{c}>20 \text { years } \\
(N=27)\end{array}$ \\
\hline & $\%$ & $\%$ & $\%$ \\
\hline Female & 50.0 & 44.0 & 40.7 \\
\hline Femur & 61.8 & 51.2 & 63.0 \\
\hline Humerus & 14.7 & 4.9 & 7.4 \\
\hline Axial skeleton & 5.9 & 7.3 & 14.8 \\
\hline Presence of metastases at diagnosis & 8.8 & 26.8 & 25.9 \\
\hline Elevated alkaline phosphatase* & 60.7 & 63.4 & 42.3 \\
\hline Tumor size $>10 \mathrm{~cm}$ & 67.9 & 39.0 & 26.0 \\
\hline Good responders & 26.9 & 5.9 & 9.5 \\
\hline Amputation/Rotationplasty** & 74.2 & 39.4 & 34.6 \\
\hline Limb-sparing resection** & 25.8 & 60.5 & 65.4 \\
\hline Marginal surgical margine* & 22.6 & 29.7 & 32.0 \\
\hline Local recurrence & 8.8 & 7.3 & 11.1 \\
\hline Distant recurrence & 29.4 & 31.7 & 33.3 \\
\hline$\geq 15 \%$ reduction of chemotherapy & 8.8 & 21.9 & 22.5 \\
\hline
\end{tabular}

Table III. Patient Characteristics and Overall Survival (OS) ( $N=102)$

\begin{tabular}{|c|c|c|c|}
\hline Variables & $\begin{array}{l}\text { Number of } \\
\text { patients }\end{array}$ & $\begin{array}{c}\text { 5-year oS } \\
(\%)\end{array}$ & p-Value ${ }^{a}$ \\
\hline \multicolumn{4}{|l|}{ Sex } \\
\hline Female & 47 & 56.5 & - \\
\hline Male & 55 & 49.1 & 0.156 \\
\hline \multicolumn{4}{|l|}{ Age } \\
\hline$\leq 14$ years & 34 & 70.6 & - \\
\hline $15-19$ years & 41 & 52.5 & 0.113 \\
\hline$>20$ years & 27 & 33.3 & 0.004 \\
\hline \multicolumn{4}{|l|}{ Alkaline phosphatase* } \\
\hline Normal & 39 & 64.1 & - \\
\hline Elevated & 55 & 51.9 & 0.115 \\
\hline \multicolumn{4}{|l|}{ Lactate dehydrogenase* } \\
\hline Normal & 11 & 54.4 & - \\
\hline Elevated & 74 & 56.8 & 0.780 \\
\hline \multicolumn{4}{|l|}{ Maximal tumor length* } \\
\hline$<10 \mathrm{~cm}$ & 44 & 56.8 & - \\
\hline$>10 \mathrm{~cm}$ & 46 & 51.1 & 0.294 \\
\hline \multicolumn{4}{|l|}{ Primary site } \\
\hline Extremities & 93 & 56.5 & - \\
\hline Axial & 9 & 22.2 & 0.003 \\
\hline \multicolumn{4}{|l|}{ Location* } \\
\hline Humerus & 7 & 71.4 & - \\
\hline Tibia & 20 & 60.0 & 0.53 \\
\hline Femur & 59 & 54.2 & 0.47 \\
\hline \multicolumn{4}{|l|}{ Histologic subtype* } \\
\hline Chondroblastic & 11 & 54.5 & - \\
\hline Osteoblastic & 63 & 51.6 & 0.975 \\
\hline Fibroblastic & 6 & 50.0 & 0.822 \\
\hline \multicolumn{4}{|l|}{ Stage } \\
\hline Local & 81 & 58.8 & - \\
\hline Metastatic & 21 & 33.3 & 0.011 \\
\hline
\end{tabular}

* not all data available

a Calculated using cox regression 


\section{Adolescent patients}

The group of 41 adolescents (aged 15 to 19 years) included 21 patients treated in the pediatric department (mean age 15.9 years; range, 15.0 to 18.5 years) and 20 patients treated in the adult department (mean age 17.3 years; range, 15.2 to 18.7 years). The clinical characteristics of the patients did not differ between these two groups e.g. no more metastasis or difference in tumor mass was seen between these two groups (data not shown). No significant difference in outcome was seen between patients treated at the pediatric department (OS $57.9 \% \pm 1.2 \%$ ) and patients treated at the department of medical oncology (OS $47.4 \% \pm 1.2 \%, p=0.705$, Figure I).

Figure I. Overall survival in patients aged 15-19 years old

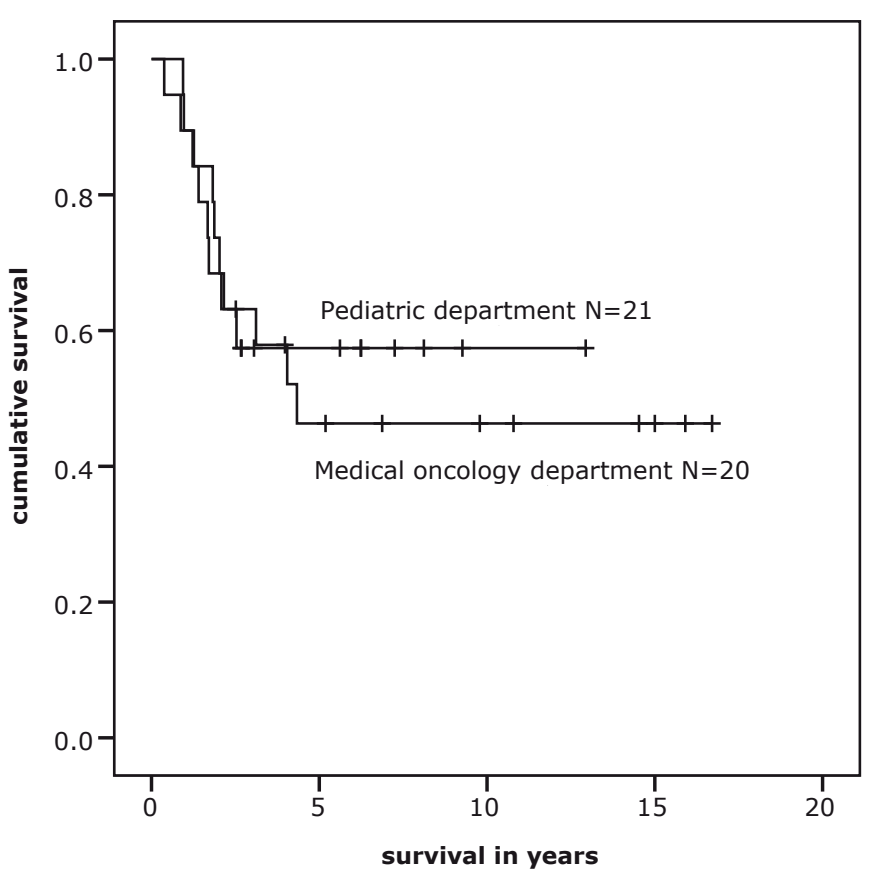

\section{Treatment}

\section{Surgery}

Concerning the surgical approach of osteosarcomas in extremities, no significant difference in 5-year DFS was seen between a limb-sparing resection and amputation (55.3\% vs. $56.2 \%, p=0.921$, Table IV). Amputation or rotationplasty were more often an option in patients $\leq 14$ years than in the two other age groups $(74.2 \%$ vs. $39.4 \%$ and $34.6 \%$ respectively, Table II). The percentages of patients with marginal surgery margins were similar in the three age groups.

Table IV. Treatment characteristics and DFS (N=102)

\begin{tabular}{|c|c|c|c|c|}
\hline Variables & & $\begin{array}{l}\text { Number of } \\
\text { patients }\end{array}$ & $\begin{array}{c}\text { 5-year DFS } \\
(\%)\end{array}$ & p Value \\
\hline \multicolumn{5}{|c|}{ Chemotherapy } \\
\hline & CIS/DOXO/HD-MTX & 20 & 63.2 & - \\
\hline & CIS/DOXO & 70 & 40.0 & 0.138 \\
\hline & Adjuvant only & 12 & 41.7 & 0.138 \\
\hline \multicolumn{5}{|c|}{ Histologic response* } \\
\hline & Good & 11 & 72.7 & - \\
\hline & Poor & 70 & 45.7 & 0.169 \\
\hline \multicolumn{5}{|l|}{ Surgery* } \\
\hline & Amputation & 47 & 55.3 & - \\
\hline & Limb-salvage & 48 & 56.2 & 0.921 \\
\hline \multicolumn{5}{|c|}{ Surgical margins* } \\
\hline & Radical & 10 & 60.0 & - \\
\hline & Wide & 49 & 51.0 & 0.669 \\
\hline & Marginal & 26 & 38.5 & 0.456 \\
\hline
\end{tabular}

* not all data available

a The significance level of $\mathrm{Chi}^{2}$ test

CIS=cisplatin, DOXO=doxorubicin, HD-MTX=high-dose methotrexate 


\section{Chemotherapy}

The 5 -year DFS of the whole population was $47.1 \% \pm 1.6 \%$. Patients $\leq 14$ years showed a better 5 -year DFS compared to their older counterparts (52.9\% vs. $47.5 \%$ in patients 15 19 years vs. $29.6 \%$ in patients $>20$ years, $p=0.05$ ).

Analyzing the different treatment regimens, the 5 -year DFS of patients treated with cisplatin and doxorubicin (CIS/DOXO) was comparable in all age groups; $40.9 \% \pm 1.8 \%$ in patients $\leq 14$ years $(\mathrm{N}=18)$ vs. $44.8 \% \pm 1.1 \%$ in patients aged $15-19$ years $(\mathrm{N}=29)$ vs. 38.9 $\% \pm 0.4 \%$ in patients aged $>20$ years $(\mathrm{N}=23)$. HD-MTX was not applied in patients older than 20 years of age because of increased toxicity of HD-MTX in elderly ${ }^{27}$; therefore, the influence of HD-MTX on the survival rate was studied in the pediatric population only. Patients younger $\leq 14$ years $(\mathrm{N}=11)$ had a DFS of $72.7 \% \pm 0.5 \%$ vs. $50 \% \pm 0.9 \%$ in patients aged $15-19$ years $(N=9)$. Furthermore, patients $\leq 14$ years had more often a good histological response to chemotherapy than older patients $(26.9 \%$ vs. $9.5 \%, p=0.058$, Table II).

\section{Treatment adjustments}

The cumulative dose of chemotherapy was reduced in 18 patients (17.6\%). Reasons for reduction of chemotherapy were the following; two patients had antracycline-induced decreased left ventricular ejection fraction, four patients had ototoxicity ( $>20 \mathrm{~dB}$ loss at 4,000 $\mathrm{Hz}$ ), severe bone marrow suppression in 10 patients and in 2 patients chemotherapy was stopped for severe psychological problems. In 14/18 patients the cumulative dose of doxorubicin was reduced with a median reduction of 30\% (range 15-80\%). Cisplatin was reduced in $16 / 18$ patients with a median reduction of $30 \%$ (range $15-50 \%$ ) of the cumulative dose. The tendency to reduce dosage due to toxicity was higher in patients older than 14 years (Table II). Treatment adjustment was not associated with a reduced 5 -years overall survival; $55.4 \%$ in patients without reduction vs. $44.4 \%$ in patients with reduction $(p=0.51)$. Significantly less patients treated at the department of pediatrics received a dose reduction (4/55 patients, $7.2 \%$ ) as compared to patients treated at the department of medical oncology (14/47 patients, $29.7 \%, p=0.005)$. Also in the group of patients aged $15-19$ years, dose reduction was more frequently applied at the department of medical oncology (4/20 patients) than at the department of pediatric oncology (1/21 patients). The presence of metastases at diagnosis was not a reason for dose reduction or dose intensification in the primary treatment.

\section{Recurrence of the disease}

In total, 41 patients (40.2\%) relapsed with a median of 15.3 months (range 2 to 101 months) after finishing chemotherapy treatment. Of these 41 patients, nine developed local recurrence and 32 relapsed with distant metastases. Recurrence rates were similar in the three different age groups (Table II). At the last time point of follow-up (median follow up time 28 months after recurrence), nine of the relapsed patients were still alive.

\section{Analysis of prognostic factors}

\section{Univariate Analyses}

On univariate analyses, older age, presence of metastases and tumors located in the axial skeleton were identified as predictors for worse overall survival (Table III). Patients $\leq 14$ years showed a significant better 5 -year OS of $70.6 \% \pm 0.8 \%$, compared to older patients aged 20 to 40 years old with an OS of $33.3 \% \pm 0.9 \%$ ( $p=0.002$, Figure II). In univariate Cox regression, with age as an isolated, continuous variable, the risk ratio was 1.030 per increasing year ( $p=0.048 ; 95 \%$ CI: $1.000-1.061$ ), indicating age is associated with survival to a significant degree.

Figure II. Overall survival by age group

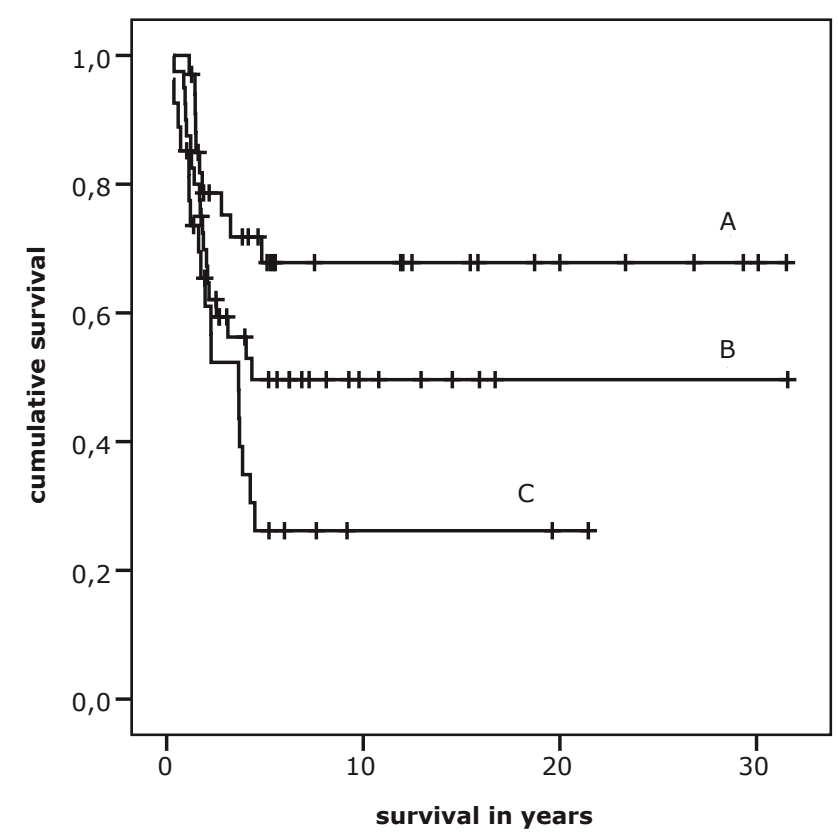

A: Age $\leq 14$ years, $N=34$, OS $70.6 \%$ ] A vs. $B p=0.11$

B vs. $C p=0.11 \quad$ A vs. $C p=0.002$

We then analyzed whether the year of treatment affected the outcome due to differences in chemotherapy and surgical techniques over the past 30 years. The year of diagnose was analyzed as continue factor for a 5 -year overall survival in a cox regression analysis which showed no difference in survival according to the year of diagnosis $(O R=0.99$; 95\% CI: 0.96-1.03; $p=0.96)$. Furthermore, we analyzed the age differences per protoco 
and treatment period. This analyzes showed that independent on period of treatment and the different regimens that were used over the past 30 years, patients $\leq 14$ years have a better survival compared to their older counterparts (Table $\mathrm{V}$ ).

Table V. Overall survival according to period of treatment and treatment regimen

\begin{tabular}{|lcc|}
\hline Treatment per periode & Age group & 5-year OS-rate (\%) \\
\hline Treatment regimen & $<14$ years & $66.7 \%$ \\
1979-1983 & $15-19$ years & $33 \%$ \\
& $20-40$ years & $0 \%$ \\
\hline $\mathbf{1 9 8 4 - 2 0 0 2}$ & $<14$ years & $57.1 \%$ \\
CIS/DOXO & $15-19$ years & $39.3 \%$ \\
& $20-40$ years & $18.2 \%$ \\
\hline $\mathbf{2 0 0 3 - 2 0 0 8}$ & $<14$ years & $75 \%$ \\
CIS/DOXO/MTX in children & $15-19$ years & $55.2 \%$ \\
CIS/DOXO in adults & $20-40$ years & $42.9 \%$ \\
\hline
\end{tabular}

CIS=cisplatin, DOXO=doxorubicin, HD-MTX=high-dose methotrexate

\section{Multivariate Analyses}

A multivariate Cox proportional hazard analysis showed that age is an independent significant prognostic factor in relationship to OS after adjustment for other significant co-variables (Table VI). Patients $\geq 20$ years of age were at greater risk of death than younger patients after adjustment for presence of metastases and tumor location in the axial skeleton $(H R=3.2 ; 95 \%$ CI: $1.5-6.9 ; p=0.004)$.

\section{Discussion}

In this retrospective, single center study we found that age above 20 years, presence of metastases and tumor location in the axial skeleton were significantly associated with decreased survival in patients with osteosarcoma. Presence of metastases at diagnosis and tumor location in the axial skeleton are generally accepted unfavorable prognostic factors ${ }^{13}$. Regarding age, however, results are contradictive. In line with our study are the results of Mankin et al. ${ }^{15}$ and Lee et al. ${ }^{16}$ studying 648 patients and 288 patients respectively, both studies showing a worse outcome with increasing age. In contrast are the studies from the Rizzoli Institute showing an unfavorable prognosis for patients aged 14 and younger ${ }^{19,20,28}$. These differences in results might be explained by the fact that in the studies by the Rizzoli Institute no patients with metastasis were included as well as no patients with osteosarcoma in the axial skeleton. In our study however, all patients were included showing significant differences in exactly these two factors within the different age groups, namely less metastases at diagnosis and less tumors located in the axial skeleton in the younger age groups. This is in concordance to the literature where the general explanation for the poor prognosis in older patients includes increased axial distribution and metastatic disease at diagnosis ${ }^{27}$. Interestingly, regardless of these associations, we found that age remained a significant prognostic factor independent on metastatic stage at diagnosis and tumor location ( $p$-value 0.004).

Another recently published study by Hartig et al. ${ }^{29}$ showed that age as a continuous variable was marginally prognostic significant. However, when patients were dichotomized by age, it was not found to be a significant prognostic variable. The chemotherapeutic protocols used in the study of Hartig et al included the use of cisplatin and doxorubicin but no HD-MTX, one of the cornerstones in the treatment of pediatric patients with osteosarcoma. In our study, we showed in an univariate analysis that outcome was not dependent on the administered regimen. Patients older than 20 years of age, however, did not receive HDMTX, thus multivariate analysis correcting for age and the regimen used was not possible. It has been earlier suggested that especially patients younger than 12 years who received HD-MTX showed a substantially improved response and survival rate ${ }^{30}$. It is therefore possible that in the younger patients the addition of HD-MTX in the treatment of osteosarcoma is beneficial and this explains the differences in survival. Addition of HD-MTX in the elderly patient, however, seems not the best option. In the study of Bacci et al. ${ }^{31}$, delayed clearance of HD-MTX resulting in severe toxicity was significantly correlated with the age of patients ( $6 \%$ for younger patients vs. $16 \%$ for patients older than 20 years: $p=0.0001$ ) For this reason HD-MTX is often not administered in patients older than 20 years as in ou hospital which however, could play an important role in long-term survival. Although in the patients older than 20 years HD-MTX was not given in our study cohort, dose adjustment due to toxicity occurred more frequently than in patients younger than 20 years. Treatment adjustments were no predictor for inferior overall survival in the multivariate analysis. Adolescents with osteosarcoma may be treated at a pediatric department or at a medical 
oncology department. Several studies (predominantly in ALL) have indicated that outcomes were better for adolescents treated at pediatric departments compared with patients treated at adult department ${ }^{18}$. To evaluate whether different treatment strategies of different departments (pediatric or adult department) is of influence regarding outcome in osteosarcoma patients, we investigated treatment results of the adolescence patients treated at our pediatric department $(\mathrm{N}=21)$ compared to adolescence patients at our adult department $(N=20)$. Although the number of patients studied in the adolescence group is small, our results suggest that there is no difference in outcome regarding treatment at the adult oncology department versus pediatric oncology ( $58 \%$ vs. $47 \%, p=0.7)$.

Thecurrentstudyshowsthatpatientsyoungerthan 14 yearshaveasignificantly betterprognosis than patients older than 20 years. Although patients with osteosarcoma aged between 15-19 years had an inferior survival compared to patients $\leq 14$ years ( $52 \%$ vs. $70.6 \%$, respectively) this was not significantly different. These results however, show that there is a clear tendency for decreased survival with increasing age. Different tumor biology in younger patients and/or different ability to cope with high-dose drugs compared to older patients might explain this difference, however, no data are yet available. With a better understand ing of the biology of osteosarcoma and more insight in age-related changes in the pharmacology of drugs (e.g. pharmacogenetics), better treatment regimens might be developed

\section{References}

Mirabello L, Troisi RJ, Savage SA. Osteosarcoma incidence and survival rates from 1973 to 2004: data from
the Surveillance, Epidemiology, and End Results Program. Cancer, 2009:115:1531-1543. Dahlin DC. Osteosarcoma of bone and a consideration of prognostic variables. Cancer Treat Rep. 1978;63:
189-192.

3 Cortes EP, Holland JF, Wang JJ, et al. Doxorubicin in disseminated osteosarcoma. JAMA. 1972;221:1132-
1138 .

Jaffe $N$, Paed $D$, Farber $\mathrm{S}$, et al. Favorable response of metastatic osteogenic sarcoma to pulse high-dose
methotrexate with citrovorum rescue and radiation therapy. Cancer. 1973;31:1367-1373.

5 Smith MA, Ungerleider RS, Horowitz ME, et al. Influence of doxorubicin dose intensity on response and
outcome for patients with osteogenic osteosarcoma and Ewing's sarcoma. I Natl Cancer Inst. 2001;83:14601470.

6 Lewis IJ, Nooij MA, Whelan J, et al. Improvement in histologic response but not survival in osteosarcoma
patients treated with intensified chemotherapy: a randomized phase III trial of the European Osteosarcoma patients treated with intensified chemotherapy: a
Intergroup. J Natl Cancer Inst. 2007; $99: 112-128$.

7 Graf N, Winkler K, Betlemovic $\mathrm{M}$, et al. Methotrexate pharmacokinetics and prognosis in osteosarcoma. J Clin

Delepine $N$, Delepine $G$, Bacci $G$, et al. Influence of methotrexate dose intensity on outcome of patients with
high grade osteogenic osteosarcoma. Analysis of the literature. Cancer. 1996; $78: 2127-2135$.

9 Goldsby R, Burke C, Nagarajan R, et al. Second solid malignancies among children, adolescents, and young adults diagnosed with malignan
Cancer. 2008;113:2597-2604

Mansky P, Arai A, Stratton P, et al. Treatment Late Effects in long-term survivors of pediatric sarcoma. Pediatr
Blood Cancer. 2007;48:192-199.

11 Pui CH, Evans WE. Acute lymphoblastic leukemia. N Engl J Med. 1998;339:605-615.

12 Razzouk BI, Estey E, Pound S, et al. Impact of age on outcome of pediatric acute myeloid leukemia. Cancer.

Bielack SS, Kempf-Bielack $\mathrm{B}$, Delling $\mathrm{G}$, et al. Prognostic factors in high-grade osteosarcoma of the extrem-
ities or trunk: an analysis of 1,702 patients treated on neoadjuvant cooperative osteosarcoma group protoities or trunk: an analysis of 1,702 pat
cols. J Clin Oncol. 2002;20:776-790.

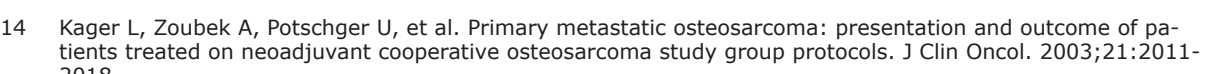

Mankin HJ, Hornicek FJ, Rosenberg AE, et al. Survival data for 648 patients with osteosarcoma treated at one
institution. Clin Orthop Relat Res. 2004:429:286-291.

Lee JA, Kim MS, Kim DH, et al. Risk stratification based on the clinical factors at diag
to the survival of localized osteosarcoma. Pediatr Blood Cancer. 2009:52:340-345. 17 Fuchs N, Bielack SS, Epler D, et al. Long-term results of the co-operative German-Austrian-Swiss osteosar-
coma study group's protocol cosS-86 of intensive multidrug chemotherapy and surgery for osteosarcoma of the limbs. Ann Oncol. 1998; $9: 893-899$

18 Ferrari S, Bertoni F, Mercuri M, et al. Predictive factors of disease-free survival for non-metastatic osteosar-
coma of the extremity: an analysis of 300 patients treated at the Rizzoli Institute. Ann Oncol. 2001; 12:1145-

Meyers PA, Heller G, Healey J, et al. Chemotherapy for non-metastatic osteogenic sarcoma. The Memoria
Sloan-Kettering experience. J Clin Oncol. 1992; 10:5-15.

20 Bacci $G$, Longhi A, Versari M, et al. Prognostic factors for osteosarcoma of the extremity treated with neo-
adjuvant chemotherapy: 15 -year experience in 789 patients treated at a single institution. Cancer. 2005; adjuvant chemoth $1061154-1161$.

21 Boissel N, Auclerc M-F, Lhéritier $V$, et al. Should adolescents with acute Iymphoblastic leukemia be treated as old children or $y 0$ 2003;21:774-780.
20s,

22 Rosen G, Caparros B, Huvos AG, et al. Preoperative chemotherapy for osteogenic sarcoma: selection of postoperatieve adjuvant chemotherapy based on the response of the primary tumor to preoperative chemo-
therapy. Cancer. 1982:49:1221-1230.

23 Enneking WF, Spanier MD, Goodman MA. A system for the surgical staging of musculoskeletal sarcoma. Clin

24 Kaplan EL, Meier P. Nonparametric observations from incomplete observations. J Am Stat Assoc. 1958;53:

25 Cox DR. Regression models and life tables. J Roy Stat Soc. 1972:34:187-220. 
26 Bieling $\mathrm{P}$, Rehan $\mathrm{N}$, Winkler $\mathrm{P}$, et al. Tumor size and prognosis in aggressively treated osteosarcoma. J Clin Oncol. 1996;14:848-858.

27 Longhi A, Errani C, Gonzales-Arabio D, et al. Osteosarcoma in patients older than 65 years. J Clin Oncol. $2008 ; 26: 5368-5373$.

28 Bacci G, Longhi A, Fagioli F, et al. Adjuvant and neoadjuvant chemotherapy for osteosarcoma of the extremities: 27 year experience at Rizzoli Institute, Italy. Eur J Cancer. 2005;41:2836-2845.

29 Hartig MT, Lally KP, Andrassy RJ, et al. Age as a prognostic factor for patients with osteosarcoma: an analysis of 438 patients. J Cancer Res Clin Oncol. 2010;4:561-570.

30 Rosen G, Marcove RC, Caparros B, et al. Primary osteogenic sarcoma: the rationale for preoperative chemotherapy and delayed surgery. Cancer. 1979;43:2168-2177.

31 Bacci G, Ferrari S, Longhi A, et al. Delayed methotrexate clearance in osteosarcoma patients treated with multiagent regimens of neoadjuvant chemotherapy. Oncol Rep. 2003;10:851-857. 


\section{CHAPTER 3}

\section{Survival trends and long term toxicity in pediatric patients with osteosarcoma}

Melanie M. Hagleitner

Eveline S.J.M. de Bont

D. Maroeska W.M. te Loo 


\section{Abstract}

\section{Background}

This study was conducted to investigate the clinical characteristics and treatment results of osteosarcoma in pediatric patients during the past 30 years. Trends in survival rates and long-term toxicity were analyzed.

\section{Methods}

In total, 130 pediatric patients under the age of 20 years with newly diagnosed, primary localized or metastatic high-grade osteosarcoma were analyzed regarding demographic, treatment-related variables, long-term toxicity and survival data.

\section{Results}

Comparison of the different time periods of treatment showed that the 5-year OS improved from $58.6 \%$ for children diagnosed during $1979-1983$ to $78.6 \%$ for those diagnosed during 2003-2008 $(p=0.13)$. Interestingly, the basic treatment agents including cisplatin, doxorubicin and methotrexate remained the same. Treatment reduction due to acute toxicity was less frequent in patients treated in the last era $(7.1 \%$ vs. $24.1 \%$ in patients treated in $1979-1983 ; p=0.04)$. Furthermore, late cardiac effects and secondary malignancies can become evident many years after treatment and can have profound impact on cancer survivors.

\section{Conclusion}

We elucidate the prevalence of toxicity to therapy of patients with osteosarcoma over the past 30 years. The overall improvement in survival may in part be attributed to improved supportive care allowing regimens to be administered to best advantage with higher tolerance of chemotherapy and therefore less chemotherapy related toxicity.

\section{Introduction}

Osteosarcoma comprises $5 \%$ of all pediatric malignancies and is the most common primary bone cancer in children and adolescents. It is a highly aggressive tumor that usually involves the metaphysis of long bones and metastasizes primarily to the lung. Before the 1970s, the prognosis for patients with high-grade osteosarcoma was poor, with long-term survival rates of less than $20 \%{ }^{1}$. Advances in adjuvant and neoadjuvant chemotherapy have improved the 5 -year disease-free survival (DFS) to more than $60 \%{ }^{2}$. Since then different attempts have been made to further improve prognosis. However, it has been demonstrated that neither dose-intensification nor addition of newer agents does improve survival ${ }^{3-6}$. Furthermore, patients that survive experience prolonged periods of rehabilitation after long periods of chemotherapeutic treatment and after often disabling surgery. Although the long term toxicities have not been assessed completely, cardiotoxicity already emerged as a significant price of cure for survivors of osteosarcoma patients ${ }^{7}$. Monitoring trends in survival and the long term toxicities is essential to enhance current treatment regimens. To gain more insight in the young patients, as osteosarcoma is predominantly being a disease that affects young patients, we investigate survival trends in this patient group and subsequently analyzed the occurrence of long term toxicity over the last three decades.

\section{Material and Methods}

\section{Patients}

In total 130 pediatric patients were consecutively treated between 1979 to 2008 in two different centers; Radboud University Nijmegen Medical Center and University Medical Center Groningen. All patients under the age of 20 years with newly diagnosed, primary localized or metastatic high-grade osteosarcoma were evaluated. Complete clinical and pathologic records, and appropriate data regarding follow-up were present. Informed consent was obtained for all patients.

\section{Treatment}

Information from the patients' medical charts about cumulative dosage of treatment, treatment response (good responders defined as $<10 \%$ vital cells after neoadjuvant therapy), reduction of chemotherapy (defined as $>15 \%$ reduction of planned chemotherapy) and surgical approach were collected. The overall period was split into sub-periods relating to the major changes in protocol (1979-1983, 1984-2002 and 2003-2008). In the first era from 1979-1983 a T10-based multidrug regimen consisting of six cycles of doxorubicin $30 \mathrm{mg} / \mathrm{m}^{2}$ daily for $2-3$ consecutive days, six cycles of cisplatin $120 \mathrm{mg} / \mathrm{m}^{2}$ administered by repeated 6 hours infusions, $8-12$ cycles of high-dose methotrexate (MTX) $8-12 \mathrm{mg} / \mathrm{m}^{2}$ 
with appropriate folinic acid rescue and 1-2 cycles of Bleomycine, Cyclophosphamide and D-Actinomycine (BCD) was used. From 1984-2002 trials with control arm treatment of six cycles of doxorubicin $25 \mathrm{mg} / \mathrm{m}^{2}$ daily for 3 days in combination with cisplatin $100 \mathrm{mg} / \mathrm{m}^{2}$ as a continuous infusion were used. The comparison arm consisted of only four doxorubicin/ cisplatin courses and additionally 4 courses high dose MTX $4 \mathrm{mg} / \mathrm{m}^{2}$ with appropriate folinic acid rescue. The last era from 2003-2008 patients were treated with six cycles of doxorubicine $25 \mathrm{mg} / \mathrm{m}^{2}$ daily for 3 days, four cycles of cisplatin $120 \mathrm{mg} / \mathrm{m}^{2}$ as a continuous infusion and 12 cycles of high-dose MTX $8-12 \mathrm{~g} / \mathrm{m}^{2}$ with appropriate folinic acid rescue.

\section{Survival}

Overall survival (OS) and disease-free survival (DFS) were estimated using the Kaplan-Meier method ${ }^{8}$. OS was defined as the interval between diagnosis and death from any cause. Patients alive at the date of last follow-up were censored at that time point. DFS was defined as the interval between diagnosis and disease progression/recurrence. Patients without disease recurrence at the date of last follow-up were censored at that date. Cox's proportional hazard regression analysis was used to determine significance of differences in survival curves. Confidence intervals were calculated at the $95 \%$ level. We evaluated gender, location, stage, treatment, treatment response, toxicity-induced reduction of chemotherapy as prognostic factors. Associations between outcome and potential predictors were evaluated with the Fisher's exact test for categorical variables. Statistical analysis was performed using SSPS software (version 16.0, SSPS Inc, Chicago, IL).

\section{Long term toxicities}

Long term toxicities after chemotherapeutic treatment was evaluated regarding development of secondary malignancies, cardiotoxicity and ototoxicity. The length of time at risk for the secondary malignancy neoplasm (SMN) was calculated from the date of diagnosis of primary osteosarcoma until the date of diagnosis of the second malignancy. The cumulative incidence rate of SMN was calculated based on the method of Gray ${ }^{9}$. For the analysis of cardiac toxicity resulting from doxorubicin therapy, we included surviving patients who underwent serial echocardiographic evaluations before, during and at least one follow-up echocardiogram after the initial treatment with doxorubicin. An echocardiogram at the end of chemotherapy was recommended annually for 5 years. Data concerning the fractional shortening (FS) value were collected. Cardiac dysfunction was defined as a decrease in FS $<28 \%$ or a reduction of more than $10 \%{ }^{10}$. Hearing loss, a complication of cisplatin was defined according to Brock Criteria ${ }^{11}$ (grade $0:<40 \mathrm{~dB}$ at all frequencies; grade1: $\geq 40 \mathrm{~dB}$ at 8000 $\mathrm{Hz}$; grade 2: $\geq 40 \mathrm{~dB}$ at $4000 \mathrm{~Hz}$; grade3: $\geq 40 \mathrm{~dB}$ at $2000 \mathrm{~Hz}$; grade $4: \geq 40 \mathrm{~dB}$ at 1000 $\mathrm{Hz}$ ). For this analysis only survivors were included with a baseline audiogram and an audiogram at the end of treatment.

\section{Results and Discussion}

\section{Patients}

The median age at diagnosis was 14.4 years (range 4.5 to 19.9 years). There were 72 male patients ( $55.4 \%$; median age 14.8 years; range, 6.3 to 19.9 years) and 58 female patients (44.6\%; median age 13.9 years; range, 4.5 to 19.1 years). At diagnosis, 29 patients $(22.3 \%)$ had metastases and in 9 patients $(6.6 \%)$ the tumor was located in the axial skeleton.

\section{Survival}

Overall survival

At a follow-up ranging between 4 and 33 years (median 8.9 years), 51 patients (39\%) died. Of all deaths, 45 were directly related to osteosarcoma with 16 deaths due to progressive disease, 8 due to local recurrence and 21 after the development of distant metastases. The development of recurrent disease was predictive of death. Incidence of recurrences was relatively stable after 5 years with only 3 patients developing disease recurrence more than 5 years after diagnosis. Of the remaining 6 patients that died, 4 patients developed a SMN which was fatal, and 2 patients passed away due to antracyclin-induced cardiomyopathy. The 5 -year OS of the whole population was $66.9 \% \pm 0.15$, the 10 -year OS was $64.6 \%$ \pm 0.23 and the 20 -year OS was $62.3 \% \pm 0.82$. These findings are in concordance with earlier reports in the literature ${ }^{12,13}$. In total, 60 patients had a follow-up longer than 5 years, 38 patients were followed for more than 10 years and a group of 20 patients was followed beyond 20 years. Comparison of the different time periods of treatment showed that the 5 -year OS improved from $58.6 \%$ for children diagnosed during $1979-1983$ to $78.6 \%$ for those diagnosed during 2003-2008 ( $p=0.13$; Figure I). The distribution of known prognostic factors, such as metastatic disease at diagnosis, tumor location and good histologic response to neoadjuvant chemotherapy was comparable regarding the different time periods (Table I). 
Figure I. 5-year overall survival in Pediatric patients with osteosarcoma over three decades

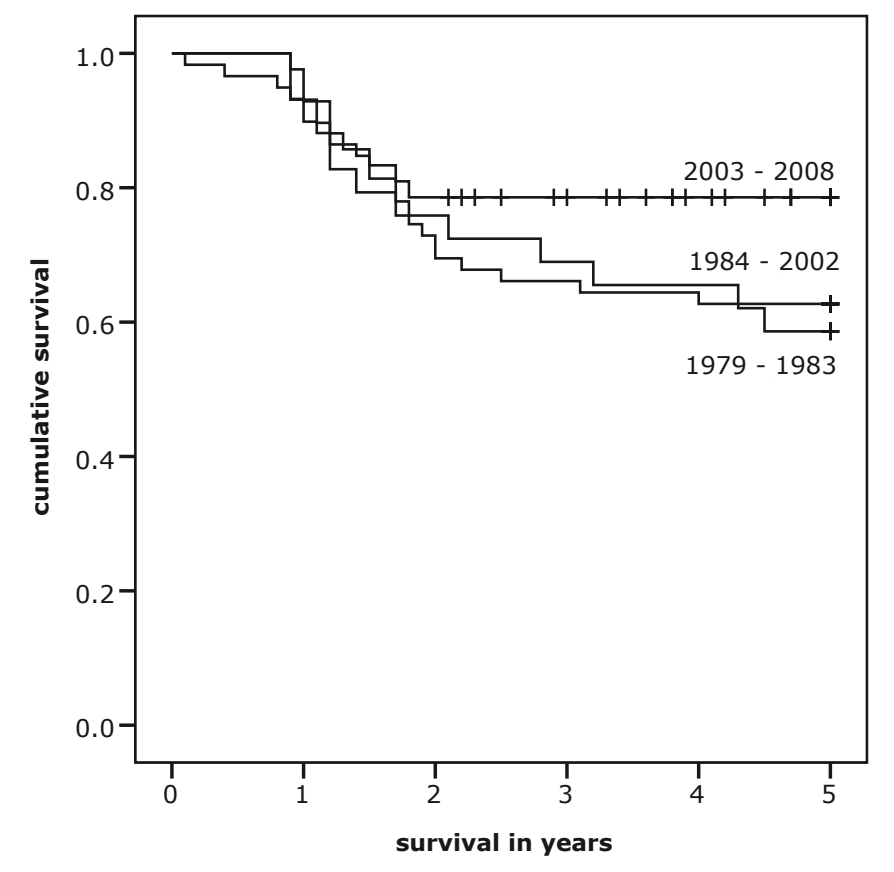

Table I. Distribution of prognostic factors over three decades

\begin{tabular}{|lcccc|}
\hline Prognostic factor (N) & $\mathbf{1 9 7 9 - 1 9 8 3}$ & $\mathbf{1 9 8 4 - 2 0 0 2}$ & $\mathbf{2 0 0 3 - 2 0 0 8}$ & $\mathbf{P}$ \\
& $\mathrm{N}=\mathbf{2 9}$ & $\mathrm{N}=59$ & $\mathrm{~N}=\mathbf{4 2}$ & \\
Metastases at diagnosis & $24.1 \%(7)$ & $25.4 \%(15)$ & $16.7 \%(7)$ & 0.58 \\
Tumor in axial skeleton & $6.9 \%(2)$ & $8.5 \%(5)$ & $4.8 \%(2)$ & 0.51 \\
Good histologic response & $24.1 \%(7)$ & $23.7 \%(14)$ & $35.7 \%(15)$ & 0.55 \\
$>$ >15\% reduction due to toxicity & $24.1 \%(7)$ & $20.3 \%(12)$ & $7.1 \%(3)$ & 0.04 \\
\hline
\end{tabular}

\section{Disease free survival}

The 5 -year DFS of the whole group was $59.2 \% \pm 0.18$. Patients with non-metastatic osteosarcoma disease showed a DFS of $65.3 \%$ vs. $37.9 \%$ in patients with metastases at diagnosis. During the past 3 decades the DFS of patients with metastases increased from $28.6 \%$ in $1979-1983,40.0 \%$ in $1984-2002$ to $42.9 \%$ in $2003-2008$. The same trend is seen in patients with bad histologic response to neoadjuvant chemotherapy: 5-year DFS increased from $30 \%$ in $1979-1983,57.6 \%$ in $1984-2002$ to $65.4 \%$ in $2003-2008$. Comparison of DFS in patients with axial involvement was not possible due to a small group.

\section{Treatment}

\section{Chemotherapeutic treatment}

In the first era, surgical resection was performed after 4 weeks of neoadjuvant chemotherapy, after 1984 resection was performed in the $10^{\text {th }}$ week of induction therapy. Good histologic response to neoadjuvant chemotherapy was observed in $13.3 \%$ of patients treated with cisplatin and doxorubicin and in $29.4 \%$ of patients additionally treated with MTX $(p=0.083)$. Although, an increased rate of good histologic responders was seen in the group additionally treated with MTX, comparing the regimen arms with and without MTX no evident difference was seen in 5-year OS $(H R=0.75,95 \% C I=0.39-1.27 ; p=0.24)$ or 5 -year DFS (HR=0.75, 95\% CI=0.43-1.29; $p=0.29$ ). The effect of high-dose MTX in the treatment of osteosarcoma is not unambiguously proven in the literature. Several studies have shown a relationship between peak serum concentrations of MTX and improved histologic response ${ }^{14-16}$. However, a Cochrane systematic review comparing the effectiveness of MTX was unable to make clear conclusions due to the lack of randomized controlled trials using high-dose MTX as the only difference between the intervention and control group ${ }^{17}$.

\section{Toxicity related treatment reduction}

Chemotherapy was reduced due to severe bone marrow depression $(N=6)$, renal toxicity $(\mathrm{N}=3)$, ototoxicity $(\mathrm{N}=3)$, cardiotoxicity $(\mathrm{N}=3)$ and adverse effects to MTX, like MTX encephalopathy $(\mathrm{N}=1)$ or allergic reactions to $\mathrm{MTX}(\mathrm{N}=3)$. Interestingly, treatment reduction due to acute toxicity is less frequent in patients treated between 2003-2008 than in patients treated between 1984-1998 and in patients treated between 1979-1983 ( $p=0.04$, Table I). 


\section{Surgical treatment}

Analysis of surgical treatment method showed a rise from $26.9 \%$ of patients with limb salvage treatment in $1979-1983$ to $69 \%$ in 2003-2008. The 5-year DFS rates were $57.8 \%$ for limb salvage treatment and $66.1 \%$ for amputation ( $H R=1.35,95 \% C I=0.76-2.39$, $\mathrm{p}=0.30$ ). The advancement of patients with limb salvage treatment could be expected considering that amputation patients might have had more advanced disease. A slightly but not significant improvement of DFS was seen in both surgical methods over the past three decades (data not shown).

\section{Long term toxicity}

\section{Secondary malignancy neoplasm}

In total, seven patients $(5.3 \%)$ developed a second malignancy, with a median latency from original diagnosis to second malignancy of 12 years (range 2.4 to 23 years). Second malignancies were 2 patients with acute lymphatic leukemia, 4 patients with breast cancer and one patient with gastric adenocarcinoma. Of the 7 patients who developed SMN, 2 patients had a history of Li-Fraumeni syndrome. Both patients developed SMN within 5 years after treatment of osteosarcoma. The 5- and 10-year estimated cumulative SMN incidence rates were $1.5 \% \pm 1.1 \%$ and $3.1 \% \pm 1.3 \%$, respectively, which is similar to earlier studies in survivors of osteosarcoma ${ }^{18,19}$. Overall, the incidence ratio of secondary malignancies in long term survivors of osteosarcoma is much less compared to other malignancies, e.g. Hodgkin disease ${ }^{20}$. However, the cohort has been followed for a median of 8.9 years, and it is likely that with increasing follow-up, SMN will emerge, due to a latency of 10 to 15 years.

\section{Cardiotoxicity}

In 46 patients sufficient data concerning baseline and follow-up echocardiogram was available. No patient had clinically evident cardiac disease before chemotherapy. Before starting therapy with doxorubicin the median FS value was $36 \%$. In 8 patients the FS decreased $<28 \%$ during therapy, with a median change in the percent FS value of $4 \%$ which is in concordance with earlier studies on this subject ${ }^{21}$. The long-term implications of doxorubicin-associated echocardiographic abnormalities are not fully understood, but at least one study suggests that echocardiographic abnormalities are progressive over time ${ }^{22}$. In our cohort, 3 patients with decreased FS during therapy remained to have cardiac dysfunction after finishing primary treatment. In total, 11 out of 46 patients developed cardiac dysfunction in the follow-up period. The median frequency of cardiac evaluation in the first five years was three (range 1 to 5 evaluations). The median latency of cardiac effects after finishing primary therapy was 219 days (range 30 days to 28.2 years). Six out of 11 patients with cardiac dysfunction experienced symptomatic heart failures; 4 of them more than 5 years after primary treatment and regular controls. Two patients died of cardiomyopathy and 3 of metastatic disease, which occurred after the diagnosis of cardiomyopathy. Affected patients were predominantly treated in the first two decades of our study realizing that the last cohort treated might not had enough follow-up to adequately evaluate this late squeal. All patients received a mean cumulative doxorubicin dose of $450 \mathrm{mg} / \mathrm{m}^{2}$.

\section{Ototoxicity}

Cisplatin associated hearing loss develops in the period cisplatin is administered and it has already been observed in patients treated with a single dose of $50 \mathrm{mg} / \mathrm{m}^{223}$. However, it is unlikely to improve over time and as even mild hearing loss can have considerably impact on the social-emotional development of a child ${ }^{24}$ it is considered to be one of the late effects in the treatment of osteosarcoma patients. Of the 79 survivors in our study, 62 $(78.5 \%)$ had an audiogram at the end of treatment. Pre-existing hearing loss was not reported in one of these patients. Of the 62 survivors with an audiogram, 31 patients (50\%) had no hearing loss, 23 patients (37.1\%) were Brock grade I and 7 patients $(11.1 \%)$ developed severe hearing loss (Brock grade 3 and 4). All patients with severe hearing loss required hearing aids. No difference was seen in cumulative dosage of cisplatin comparing patients with severe hearing loss (cumulative dosage: $407 \mathrm{mg} / \mathrm{m}^{2}$ ) versus patients with grade 0,1 or 2 hearing loss (cumulative dosage: $442 \mathrm{mg} / \mathrm{m}^{2}, \mathrm{p}=0.55$ ). Furthermore, no difference in prevalence of severe ototoxicity was seen between the drug regimens using either $600 \mathrm{mg} / \mathrm{m}^{2}$ or $480 \mathrm{mg} / \mathrm{m}^{2}$ cisplatin $(p=0.25)$ or continuous infusion versus repeated 6 hours infusions $(p=0.76)$. 
Like other studies in childhood cancer ${ }^{25}$ we showed an improved survival of patients with osteosarcoma over the past decades. This improvement is seen in all subgroups irrespective of known prognostic factors like metastases at diagnosis or histological response to neoadjuvant therapy. This increase in childhood cancer survival is often attributed to more effective treatment strategies. However, in our study the treatment agents and dosage basically remained the same over the past 30 years with cumulative doses of doxorubicin $450 \mathrm{mg} / \mathrm{m}^{2}$ cislpatin 480 or $600 \mathrm{mg} / \mathrm{m}^{2}$ and high-dose MTX $96-144 \mathrm{~g} / \mathrm{m}^{2}$ appropriate to age. Furthermore there was no difference in method of administration regarding doxorubicin and high-dose MTX. Cisplatin was administered as a 6 hours infusion during 1979-1983 in our study and then as continuous infusion. While continuous cisplatin infusions are observed to be considerable ototoxic ${ }^{26}$, we found no difference in our study. Generally, other factors like empiric use of broad spectrum antibiotics in case of neutropenic fever, pain management, antiemetics, nutrition, blood banking and improved pediatric intensive care might have improved the acceptability of the current treatment. On the other hand, with an increasing number of cancer survivors detection of late effects of osteosarcoma therapy is required. In this study we show that late cardiac effects and SMN can become evident many years after treatment and can have profound impact on cancer survivors.

In conclusion, these data elucidate the prevalence of toxicity to therapy in children with osteosarcoma. Further studies concerning the management of long-term toxicities are needed to finally improve survival rates in patients with osteosarcoma.

\section{References}

1 Bacci G, Picci P, Ferrari S, et al. Primary chemotherapy and delayed surgery for nonmetastatic osteosarcoma of the extremities: Results in 164 patients preoperatively

2 Bielack S, Carrle D, Hardes J, et al. Bone tumors in adolescents and young adults. Current Treat Opt Oncol. 2008;9:67-80.

3 Eselgrim $\mathrm{M}$, Grunert $\mathrm{H}$, Kühne T, et al. Dose intensity of chemotherapy for osteosarcoma and outcome in the

4 Lewis IJ, Weeden S, Machin D, et al. Received Dose and Dose-Intensity of Chemotherapy and Outcome in

5 Bacci G, Forni C, Ferrari S, et al. Neoadjuvant chemotherapy for osteosarcoma of the extremity: intensification of preoperative treatment does not increase the rate of good histologic response to the primary tumor or
improve the final outcome. J Pediatr Hematol Oncol. 2003;25:845-853.

$6 \begin{aligned} & \text { Ferrari S, Palmerini E.Adjuvant and neoadjuvant combination chemotherapy for osteogenic sarcoma. Current } \\ & \text { Opinion in Oncology. 2007;19:341-346. }\end{aligned}$

7 Bacci $G$, Ferrari $S$, Bertoni $F$, et al. Long-term outcome for patients with nonmetastatic osteosarcoma of the extremity treated at the istituto ortopedico rizzoli according to the istituto ortopedico rizzoli/osteosarcoma-2
protocol: an updated report. 3 Clin Oncol. 2000;18:4016-4027.

8 Kaplan EL, Meier P. Nonparametric observations from incomplete observations. J Am Stat Assoc. 1958;53:

9 Gray RJ. A class of K-sample tests for comparing the cumulative incidence of a competing risk. Am Stat.

10 Kimball TR, Michelfelder EC. Echocardiography, in Moss \& Adams's "Heart Disease in Infants, Children, and
Adolescents", 8th edition. Philadelphia: Lippincott Williams \& Wilkins; 2008, pp. 95-162.

11 Brock PR, Bellman SC, Yeomans EC, et al. Cisplatin ototoxicity in children: a practical grading system. Med
Pediatr Oncol. 1991; 19:295-300.

12 Bielack $S S$, Kempf-Bielack $B$, Delling $G$, et al. Prognostic factors in high-grade osteosarcoma of the extrem-
ities or trunk: an analysis of 1,702 patients treated on neoadjuvant cooperative osteosarcoma study group ities or trunk: an analysis of 1,702 patients
protocols. J Clin Oncol. 2002;20:776-790.

Nagarajan $R$, Kamruzzaman A, Ness KK, et al. Twenty years of follow-up of survivors of childhood osteosarco-
ma: a report from the Childhood Cancer Survivor Study. Cancer. 2011; 117:625-634.

14 Delapine $N$, Delapine $G$, Jasmine $C$, et al. Importance of age and methotrexate dosage: prognosis in children

15 Bacci G, Ferrari S, Delapine $\mathrm{N}$, et al. Predictive factors of histological response to primary chemotherapy in osteosarcoma of the extremity: study of 272 patients preop
doxorubicin and cisplatin. J Clin Oncol. 1998; 16:658-663.

16 Graf $\mathrm{N}$, Winkler K, Betlemovic M, et al. Methotrexate pharmacokinetics and prognosis in osteosarcoma. J Clin
Oncol. 1994;12:1443-1451.

17 van Dalen EC, van As JW, de Camargo B. Methotrexate for high-grade osteosarcoma in children and young adults. Cochrane Database Syst Rev. 2011;5:CD006325.

18 Pratt CB, Meyer WH, Luo X, et al. Second malignant neoplasms occurring in survivors of osteosarcoma.
Cancer. 1997;80:960-965.

Aung $L$, Gorlick RG, Shi W, et al. Second malignant neoplasms in long-term survivors of osteosarcoma. Me-
morial Sloan-Ketting Cancer Center Experience. Cancer. 2002;95:1728-1734. Neglia JP, Friedman DL, Yasui Y, et al. Second malignant neoplasms in five-year survivors of childhood
cancer: childhood cancer survivor study. I Natt Cancer Inst. 2001;93:618-629.

21 Lipshultz SE, Sanders SP, Colan SD, et al. Monitoring for anthracycline cardiotoxicity. Pediatrics. 1994;93: 22 Steinherz $L$ J, Steinherz $P G$, Tan $C T$, et al. Cardiac toxicity 4 to 20 years after completing anthracycline thera-
py. JAMA. 1991;266:1672-1677.

23 Stavroulaki P, Apostolopoulos N, Segas J, et al. Evoked otoacoustic emissions-an approach for monitoring
cisplatin induced ototoxicity in children. Int J Pediatr Otorhinolaryngol. 2001; $59: 47-57$.

24 Li Y, Womer RB, Silber JH. Predicting cisplatin ototoxicity in children: the influence of age and the cumulative 
25 Pui CH, Gajjar AJ, Kane JR, et al. Challenging issues in pediatric oncology. Nat Rev Clin Oncol. 2011;8:540549.

26 Lanvers-Kaminsky C, Krefeld B, Dinnesen AG, et al. Continuous or repeated prolonged cisplatin infusions in children: a prospective study on ototoxicity, platinum concentrations, and standard serum parameters. Pediatr Blood Cancer. 2006;47:183-193. 


\section{CHAPTER 4}

\section{Taqman genotyping assays can be used on decalcified and paraffin embedded tissue from patients with osteosarcoma}

Melanie M. Hagleitner

Marieke J.H. Coenen

Judith W.M. Jeuken

Uta Flucke

H.W. Bart Schreuder

D. Maroeska W.M. te Loo

Peter M. Hoogerbrugge 


\section{Abstract}

\section{Background}

In cancers like osteosarcoma with a 5-year overall survival of 50-60\%, archived histological specimens can be a useful source of biological material. However, this material generally has been decalcified and formalin-fixed for many years. In our study, we investigated whether DNA obtained from these tissues can be used for reliable single nucleotide polymorphism (SNP) genotyping.

\section{Methods}

We studied two SNPs in the drug transporter MDR1 using Taqman ${ }^{\circledR}$ SNP genotyping assays. Genotypes of the germ-line DNA derived from freshly isolated DNA of 20 surviving patients with osteosarcoma were compared with genotypes obtained from archived material from decalcified formalin-fixed, paraffin-embedded (FFPE) blocks of the same patients.

\section{Results}

Decalcified FFPE-derived DNA yielded smaller polymerase chain reaction (PCR) fragments compared to DNA extracted from peripheral blood cells, with a reliable size of $\sim 200 \mathrm{bp}$. However, we were able to evaluate each SNP in 19 of 20 cases included in this study. All successfully genotyped samples showed $100 \%$ concordance between genotypes obtained from DNA of FFPE tissue and the genotypes obtained from DNA of blood from the same patients.

\section{Conclusions}

In conclusion, we have demonstrated that decalcified FFPE tissue can be used for genetic polymorphism analysis using Taqman ${ }^{\circledR}$ allelic discrimination assays. This forms an unique opportunity to combine new insights in genetic research with historical patient cohorts.

\section{Introduction}

Osteosarcoma is the most frequent primary malignant tumor of the bone. With current treatment regimens, the 10 -year overall survival approaches $60 \%{ }^{1}$. Major progress in the therapy of patients with osteosarcoma was achieved in the early 1970s when chemotherapy was added to surgery ${ }^{2,3}$. However, survival rates have reached a plateau over the last decade, suggesting that we have reached the limits of currently applied therapy modalities. Further improvement in outcome and reduction in toxicity will require the identification and application of new prognostic factors and novel treatment strategies.

Multiple studies have suggested that outcome in different types of cancers may be affected by how rapidly and effectively an individual patient metabolizes certain chemotherapeutic agents ${ }^{4}$. Genetic variations can affect the effectiveness and toxicity of chemotherapeutic agents ${ }^{5}$. Germ line DNA is needed to identify the genetic factors underlying toxicity and efficacy of drugs that are currently used in the treatment of cancer. However, most clinical trials performed so far in cancer treatment did not routinely collect and store patient germ line DNA. Formalin-fixed paraffin-embedded tissues (FFPE) from archived clinica samples could provide a source of germ line DNA for genetic research. Specially, in cancers like osteosarcoma with a 5-year overall survival (OS) of approximately 50-60\%, archived histological specimens are often the only accessible source of biological material. However, DNA obtained from such samples may be different from the original germ line DNA extracted from white blood cells (WBCs) or buccal swabs due to several reasons: The DNA from FFPE tissues has a higher frequency of non-reproducible sequence alterations compared with germ line DNA ${ }^{6}$. It has been hypothesized that the artifacts are caused due to formalin fixation as this can lead to cross-linking between cytosine nucleotides on either strand of the DNA as well as to cross-linking between histone proteins that normally coat the DNA. In both cases DNA amplification in a polymerase chain reaction (PCR) can be unreliable. First, cross-linking of cytosine nucleotides precludes the Taq DNA to recognize the cytosine which therefore, incorporates an adenine instead of a guanine resulting in an artificial C-T or G-A mutation ${ }^{7,8}$. And second, cross-linking of histone proteins is likely to affect accessibility of the DNA by Taq DNA polymerase and the subsequent progression of the polymerase along native DNA template making the DNA inaccessible to PCR 9 . Furthermore, fixed bone tumor often requires acid decalcification prior to processing, which may cause additional DNA fragmention that potentially influences the result of subsequent SNP analysis ${ }^{10,11}$. Especially, when assays are based on relatively long amplicons (above $\sim 200$ base paires), fragmentation of DNA influences the reliability of the evaluation.

Although, previous studies have used FFPE material for molecular diagnostics in patients with osteosarcoma ${ }^{12-14}$, only one report has used decalcified FFPE material for DNA isolation. In this report DNA from decalcified FFPE samples was unsuitable for genotype analysis due to significant degradation of DNA based on decalcification ${ }^{15}$. Consequently, there are no studies reporting the successful experience of extracting DNA from decalcified FFPE 
tissue. Therefore, we analyzed whether the genotypes obtained from DNA of these tissues, were comparable with the genotypes obtained from freshly isolated DNA from saliva or peripheral blood cells from the same patients.

\section{Materials and methods}

\section{Patients and samples}

We included samples of 20 patients with primary, high-grade osteosarcoma. From each patient, germ line DNA was isolated from blood or saliva and from stored normal FFPE tissue. Collection and extraction of germ line DNA from blood or saliva samples occurred in 2008 and 2009. All these patients had an excision of the tumor between 1997 and 2008. At time of surgical resection, fresh tissue material was fixed in $4 \%$ formaldehyde, phosphate-buffered at pH 7.0 (Klinipath, Duiven, The Netherlands) for 12 to 16 hours at room temperature. Ten of the 20 tissue samples were decalcified in 10\% formic acid for approximately one week. The tissue material was then dehydrated in a Schandon Excelsior $^{T M}$ ES Tissue Processor (Thermo Fisher Scientific Inc., Breda, The Netherlands) according to the following procedure: 5.5 hours, 50\% alcohol; 4 hours, $70 \%$ alcohol; 6.5 hours, $96 \%$ alcohol; 7.5 hours, $100 \%$ alcohol; 2 times 1 hour, 100\% alcohol; 3 times 1.5 hours, $x y$ lene; 3 times 1.5 hours, paraffin; and finally embedded in paraffin. The tissue blocks were stored at room temperature. At time of DNA extraction from the FFPE blocks, the samples had been embedded in paraffin blocks for 1-11 years. From the FFPE blocks we used muscle samples $(\mathrm{N}=15)$ or other tissues $(\mathrm{N}=5)$ such as skin or lymph nodes as normal tissue source. Sample size was uniform, $1.5 \mathrm{~cm} \times 2.5 \mathrm{~cm}$. All samples were assessed by an experienced pathologist on hematoxylin/eosin stained slides before samples were used for DNA extraction. In 3 patients, the FFPE blocks contained normal (non-tumor) tissue as well as tumor tissue. In these cases, regions containing tumor cells were indicated by an experienced pathologist and removed by macro-dissection after which non-tumor germ line DNA was isolated.

\section{DNA extraction from blood and saliva}

DNA of EDTA whole blood ( $5 \times 10^{6}$ WBCs) was extracted using the QIAamp DNA Blood Mid kit (Qiagen Inc., Valencia, CA. USA), according to the manufacturers protocol. DNA of saliva $(2 \mathrm{ml})$ was collected in the Oragene saliva collection kit (DNA Genotek, Kanata, Ontario, Canada) using the Oragene DNA purification protocol as supplied by the manufacturer

\section{DNA extraction from FFPE tissue}

Paraffin embedded samples were sectioned using standard microtome techniques. Four tissue sections of $10 \mu \mathrm{m}$ were placed in $1.5 \mathrm{ml}$ tubes. The microtome was carefully cleaned with ethanol between cases to avoid contamination. Samples were incubated in $200 \mu$
TRIS buffer ( $\mathrm{NaCl}[5 \mathrm{M}]$, Tween-20[20\%], Ethanol[70\%]) for 15 minutes at $90{ }^{\circ} \mathrm{C}$. After centrifugation $(14,000 \mathrm{rpm}) 20 \mu \mathrm{l}$ Proteinase K solution was added to the pellet. Samples were incubated for 3 days in a heating block (first day at $56^{\circ} \mathrm{C}$, second and third day at $37^{\circ} \mathrm{C}$ ); every day $20 \mu$ l Proteinase $\mathrm{K}$ solution was added to the samples. After the incubation period, samples were centrifuged at $14,000 \mathrm{rpm}$ for $10 \mathrm{~min}$, the supernatant was removed. This step was repeated twice. Then, $4 \mu \mathrm{l}$ RNase $(100 \mathrm{mg} / \mathrm{ml})$ solution was added and the DNA was isolated using the DNeasy Tissue kit as described by the manufacture (Qiagen Inc., Valencia, CA. USA) with the modification that $20 \mu \mathrm{LE}$ buffer was applied twice instead of once.

\section{DNA quality control}

The nucleic acid purity was determined by a $260 / 280 \mathrm{~nm}$ and $230 / 280 \mathrm{~nm}$ optical density (OD) measurement using the NanoDrop (Thermo Scientific NanoDrop ${ }^{\mathrm{TM}} 1000$ Spectrophotometer). A qualitative size range PCR assay was performed to determine DNA quality of the normal FFPE tissue. The assay consists of 5 primer sets, that amplify products ranging from $100 \mathrm{bp}$ to $400 \mathrm{bp}$ (Table I) and has successfully been used before ${ }^{16}$.

Table I. Primer sequences for the gel electrophoresis assays

\begin{tabular}{|lcl|}
\hline Position NCBI & Forward/Reverse primer & Primer sequence 5'-3' \\
TBXAS1/X9U (388) & Forward primer & GCC CGA CAT TCT GCA AGT CC \\
TBXAS1/X9L (487) & Reverse primer & GGT GTT GCC GGG AAG GGT T \\
RAG1/X2U (610) & Forward primer & TGT TGA CTC GAT CCA CCC CA \\
RAG1/X2L (809) & Reverse primer & TGA GCT GCA AGT TTG GCT GAA \\
PLZF/X1U (11587) & Forward primer & TGC GAT GTG GTC ATC ATG GTG \\
PLZF/X1L (11886) & Reverse primer & CGT GTC ATT GTC GTC TGA GGC \\
AF4/X11U (486) & Forward primer & CCG CAG CAA GCA ACG AAC C \\
AF4/X11L (885) & Reverse primer & GCT TTC CTC TGG CGG CTC C \\
AF4/X3U (218) & Forward primer & GGA GCA GCA TTC CAT CCA GC \\
AF4/X3L (817) & Reverse primer & CAT CCA TGG GCC GGA CAT AA \\
\hline
\end{tabular}

Matching DNA from normal cells was used as a control. Genomic DNA (50 ng) was amplified using dNTPs (2 mM), PCR-buffer II, $\mathrm{MgCl}_{2}(25 \mathrm{mM}), 10$ pmol of each primer, and Taq polymerase $(2.5 \mathrm{U})$. PCR conditions were $94{ }^{\circ} \mathrm{C}$ for 10 minutes followed by 35 cycles of $92{ }^{\circ} \mathrm{C}$ for 30 seconds, $60^{\circ} \mathrm{C}$ for 40 seconds and $72{ }^{\circ} \mathrm{C}$ for 40 seconds. MilliQ was included as negative controls in all PCR experiments. PCR products were visualized using $2 \%$ ethidium bromide-stained agarose gels. The bands were visualized and recorded using the Imago (Isogen Bioscience, IJsselstein, The Netherlands). Absence of DNA bands indicat- 
ed degradation of the DNA. The degree of DNA degradation is related to the number of non-amplified bands, the largest products will disappear first.

\section{Genotyping by TaqMan ${ }^{\circledR}$ Allelic Discrimination}

Two SNPs in the drug transporter MDR1 (ABCB1), rs1045642 and rs2032582 were genotyped using Taqman ${ }^{\circledR}$ allelic discrimination assays (Table II, Applied Biosystems, Nieuwerkerk aan den IJssel, The Netherlands). Amplicon size ranges between 70-90 bp. The SNP rs2032582 is tri-allelic, meaning that three different nucleotides can occur at the same genomic location. Two assays were used to detect all three nucleotides of this SNP.

\section{Table II. Genotyping results}

\begin{tabular}{|llllccc|}
\hline Gene & SNP & Assay Name & $\begin{array}{c}\text { success rate (N) } \\
\text { non- } \\
\text { saliva }\end{array}$ & $\begin{array}{c}\text { decalcified } \\
\text { FFPE }\end{array}$ & $\begin{array}{c}\text { decalcified } \\
\text { FFPE }\end{array}$ \\
ABCB1 & rs1045642: C>T & C_7586657_20 & $20 / 20$ & $10 / 10$ & $9 / 10$ \\
& rs2032582: C>A & C_11711720C_30 & $20 / 20$ & $10 / 10$ & $10 / 10$ \\
& rs2032582: C>T & C_11711720C_40 & $20 / 20$ & $9 / 10$ & $10 / 10$ \\
\hline
\end{tabular}

The PCR reactions were performed according to the manufacturer's protocol using $10 \mathrm{ng}$ DNA in a final volume of $10 \mu \mathrm{l}$. The PCR amplification was performed using the following conditions: $12 \mathrm{~min}$ at $95^{\circ} \mathrm{C}, 50 \mathrm{cycles}$ of $15 \mathrm{sec}$ at $92^{\circ} \mathrm{C}$ and $90 \mathrm{sec}$ at $60^{\circ} \mathrm{C}$. The fluorescent signal for allelic discrimination was determined after amplification using an end-poin reading on the 7500 Fast Real-time System (Applied Biosystems). Automated allele calling was performed by allelic discrimination plots using SDS 1.4 software (Applied Biossystems). We included $5 \%$ duplicate samples in each plate as quality control.

\section{Results}

Twenty patients with osteosarcoma were included in the study. Germ line DNA, both from blood/saliva and from FFPE material was collected of all patients. The median DNA yields of the different sources are shown in Table III.

Table III. DNA yields
\begin{tabular}{|lcc|}
\hline Type of sample & N (samples) & $\begin{array}{c}\text { DNA yield }(\boldsymbol{\mu g}) \\
\text { median }(\text { range) }\end{array}$ \\
Blood $\left(\right.$ WBCs $\left.5 \times 10^{6}\right)$ & 14 & $30.0(6.6-82.0)$ \\
Saliva $(2 \mathrm{ml})$ & 6 & $55.1(8.9-134.0)$ \\
Decalcified FFPE $(4 \times 10 \mu \mathrm{m}$-slided, 1.5 à $2.5 \mathrm{~cm})$ & 10 & $9.1(0.65-19.2)$ \\
Non-decalcified FFPE $(4 \times 10 \mu \mathrm{m}$-slided, $1.5 \mathrm{à} 2.5 \mathrm{~cm})$ & 10 & $9.8(0.67-24.3)$ \\
\hline
\end{tabular}

The median yield for embedded tissues $(4 \times 10 \mu \mathrm{m}$-slices of $1.5 \times 2.5 \mathrm{~cm})$ was $9.4 \mu \mathrm{g}$ ranging from $0.65 \mu \mathrm{g}$ to $24.3 \mu \mathrm{g}$. The quality of the DNA derived from decalcified FFPE samples was comparable to the quality of the non-decalcified FFPE samples. The mean OD 260/280 ratio of non-decalcified FFPE tissue was 1.80 (range 1.39-1.91) and the mean OD 260/280 ratio of decalcified FFPE tissue was 1.77 (range 1.0-2.70)

We performed a size range PCR to determine if decalcification results in DNA degradation. Figure I shows the results of the PCR of eight samples of germ line DNA from blood/saliva compared to germ line DNA derived from FFPE tissue of the same patients. Analyses by gel electrophoresis revealed that the PCR reactions worked efficiently with DNA from blood/saliva. For DNA extracted from FFPE samples, PCR became increasingly inefficient the longer the amplicons were. In three of the four sets of decalcified FFPE samples, the ectrophoresis gel showed amplicon sizes from 100 bp to $300 \mathrm{bp}$. Bands were absent in one of the decalcified FFPE samples. The other four sets with non-decalcified normal FFPE tissue showed an amplicon size from $100 \mathrm{bp}$ to $300 \mathrm{bp}$. However, the $300 \mathrm{bp}$ fragment were less intense compared to germ line DNA derived from blood/saliva, indicating some degree of DNA degradation in the FFPE samples. 
Figure I. Results of the size-range PCR to assess the integrity of DNA from decalcified FFPE tissue

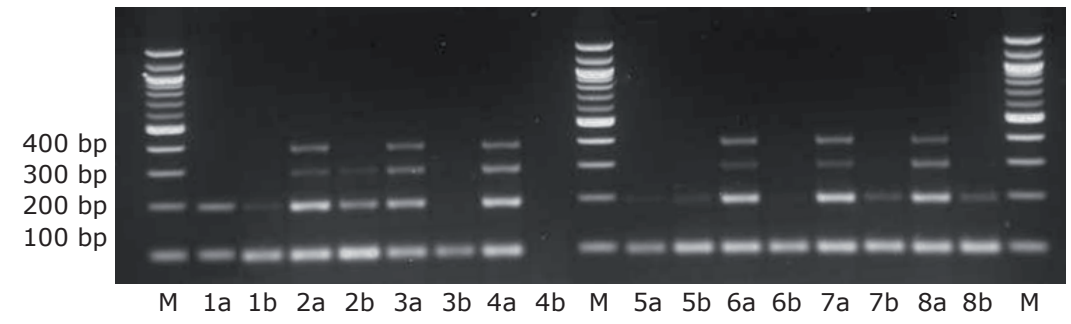

M: 100 bp size ladder

: DNA isolated from blood or saliva, b: DNA isolated from matching FFPE tissue

Lanes 1-4: DNA isolated from decalcified normal FFPE tissue;

Lanes 5-8: DNA isolated from non-decalcified normal FFPE tissue

Genotyping of two SNPs ( 3 genotype assays) in the $A B C B 1$ gene was performed on al samples. Genotyping was successful in all blood/saliva samples $(\mathrm{N}=20)$ for each assay. For one of the 10 decalcified FFPE samples, we were unable to genotype rs2032582 C>T (Table II, sample 4b). For non-decalcified samples $(\mathrm{N}=10)$, genotyping of SNP rs1045642 failed for one sample (not shown as DNA yield was too low for size range PCR). In the 18 patients in which both DNA from FFPE and blood/saliva was available, a 100\% concordance was seen comparing the genotypes obtained from DNA of the FFPE tissues to those of freshly isolated DNA from saliva or peripheral blood cells from the same patients. The two samples with unsuccessful genotyping were re-analyzed to exclude technical problems; in addition a random selection of $10 \%$ of all samples was genotyped twice as quality control. All samples replicated showed consistent genotypes.

\section{Discussion}

Genetic studies are necessary to get a better understanding of tumor biology and the treatment response in malignancy. However, in patients with osteosarcoma, in which surviva rates are about $60 \%$, representative material for genetic studies often is only present in the form of decalcified, formalin fixed specimens. So far, successful application of genetic studies on decalcified, FFPE tissues have not been reported. Reliable results with quantitative PCR have only been shown on tissue that has not been decalcified.

In a few reports, decalcified FFPE material has been used for DNA or RNA isolation. In one report DNA from decalcified FFPE tissues was unsuitable for genotype analysis due to significant degradation of DNA based on decalcification ${ }^{15}$. In two other reports, RNA extracted from decalcified samples was of poor quality and reliable for real-time PCR in only 5/35 and $6 / 40$ of the cases, respectively 17,18 .

In the present study, we demonstrate that long-term stored, decalcified FFPE tissues, can be reliably used for Taqman ${ }^{\circledR}$ genotyping assays. We were able to evaluate each SNP in 19 of the 20 cases included in this study. All successfully genotyped samples showed $100 \%$ concordance between genotypes obtained from DNA of decalcified FFPE tissue and the genotypes obtained from DNA of blood/saliva. No misclassifications were seen.

Decalcification of tissues is often necessary to soften the bone in order to achieve acceptable 3-6 $\mu \mathrm{m}$ sections of bone for histological studies. This process is carried out by removing the mineral with acids which leads to additional fragmentation of the DNA, making the recovery of nucleic acids even more challenging. In our study, we optimized our protocol with a prolonged incubation time of proteinase $\mathrm{K}$. The most important role of protease digestion is to destruct the cells, but also to de-cross link histone proteins from native DNA, which has occurred to formalin fixation ${ }^{19}$. Using this modified protocol, we were able to obtain adequate DNA in decalcified FFPE tissue suggesting that with this simple adaptation, DNA can be successfully recovered from decalcified archived tissue. Furthermore, the ability to amplify PCR products of 200 bp suggests that decalcified FFPE samples might as well be used on other genotyping methods, like high throughput genotyping using the Illumina system ${ }^{20,21}$.

In conclusion, we have demonstrated that formalin fixed and paraffin embedded tissue, whether decalcified or not, can be used for genetic polymorphism analysis using Taqman allelic discrimination assays. This forms an unique opportunity to combine new insights in genetic research with historical patient cohorts, for instance in patients with osteosarcoma. 


\section{References}

1 Bielack SS, Kempf-Bielack B, Delling G, et al. Prognostic factors in high-grade osteosarcoma of the extremities or trunk: an analysis of 1,702 patients treated on neoadjuvant cooperative osteosarcoma group protocols. J Clin Oncol. 2002;20:776-790.

2 Dahlin DC. Osteosarcoma of bone and a consideration of prognostic variables. Cancer Treat Rep. 1978;63: 189-192.

3 Cortes EP, Holland JF, Wang JJ, et al. Doxorubicin in disseminated osteosarcoma. JAMA. 1972;221:11321138.

4 Erichsen HC, Chanock SJ. SNPs in cancer research and treatment. Br J Cancer. 2004; 23;90:747-751.

5 Evans WE, Relling MV. Pharmacogenomics: Translating Functional Genomics into rational therapeutics. Science. $1999 ; 286: 487-491$.

6 Srinivasan M, Sedmak D, Jewell S. Effect of fixatives and tissue processing on the content and integrity of nucleic acids. Am J Pathol. 2002;161:1961-1971.

7 Williams C, Ponten F, Moberg C, et al. A high frequency of sequence alterations is due to formalin fixation of archival specimens. Am J Pathol. 1999;155:1467-1471.

8 Chalkley R, Hunter C. Histone-histone propinquity by aldehyde fixation of chromatin. Proc Natl Acad Sci USA. $1975 ; 72: 1304-1308$

9 O'Leary JJ, Browne G, Landers RJ, et al. The importance of fixation procedures on DNA template and its suitability for solution-phase polymerase chain reaction and PCR in situ hybridization. Hist J. 1994;26:337-346.

10 Wickham CL, Sarsfield P, Joyner MV, et al. Formic acid decalcification of bone marrow trephines degrades DNA: alternative use of EDTA allows the amplification and sequencing of relatively long PCR products. Mol Pathol. 2000;53:336.

11 Alers JC, Krijtenburg PJ, Vissers KJ et al. Effect of bone decalcification procedures on DNA in situ hybridization and comparative genomic hybridization. EDTA is highly preferable to a routinely used acid decalcifier. ] Histochem Cytochem. 1999;47:703-710.

12 Oliveira CR, Menonca BB, Camargo, et al. Classical osteoblastoma, atypical osteoblastoma, and osteosarcoma: a comparative study based on clinical, histological, and biological parameters. Clinics (Sao Paulo). 2007;62:167-174.

13 Zielenska $\mathrm{M}$, Marrano $\mathrm{P}$, Thorner $\mathrm{P}$, et al. High-resolution cDNA microarray CGH mapping of genomic imbalances in osteosarcoma using formalin-fixed paraffin-embedded tissue. Cytogenet Genome Res. 2004; 107:77-82

14 Fellenberg J, Krauthoff A, Pollandt K, et al. Evaluation of the predictive value of Her-2/neu gene expression on osteosarcoma therapy in laser-microdissected paraffin-embedded tissue. Lab Invest. 2004;84:113-121.

15 Kersting C, Agelopoulos, K, Schmidt H, et al. Biological importance of a polymorphic CA Sequence within Intron I of the Epidermal Growth Factor Receptor Gene (EGFR) in high grade central osteosarcomas. Genes Chromosomes Cancer. 2008;47:657-664.

16 van Dongen JJ, Langerak AW, Bruggemann M, et al. Design and standardization of PCR primers and protocols for detection of clonal immunoglobulin and T-cell receptor gene recombinations in suspect lymphoproliferations: report of the BIOMED-2 Concerted Action BMH4-CT98-3936. Leukemia. 2003;17:2257-2317.

17 Mangham DC, Williams A, McMullan DJ, et al. Ewing's sarcoma of bone: the detection of specific transcripts in a large, consecutive series of formalin-fixed, decalcified, paraffin-embedded tissue samples using the reverse transcriptase-polymerase chain reaction. Histopathology. 2006;48:363-376.

18 Park YK, Chi SG, Park HR, et al. Detection of $\mathrm{t}(11 ; 22)$ (q24; q12) translocation of Ewing's sarcoma in paraffin embedded tissue by nested reverse transcription-polymerase chain reaction. J Korean Med Sci. 1998; 13 : 395-399.

19 Gilbert MT, Haselkorn T, Bunce M, et al. The isolation of nucleic acids from fixed, paraffin-embedded tissues-which methods are useful when? PLoS One. 2007;2:e537.

20 Lips EH, Dierssen JW, van Eijk R, et al. Reliable high-throughput genotyping and loss-of-heterozygosity detection in formalin-fixed, paraffin-embedded tumors using single nucleotide polymorphism arrays. Cancer Res. 2005;65:10188-10191.

21 Jacobs S, Thompson ER, Nannya Y, et al. Genome-wide, high-resolution detection of copy number, loss of heterozygosity, and genotypes from formalin-fixed, paraffin-embedded tumor tissue using microarrays. Cancer Res. 2007;67:2544-2551. 


\section{CHAPTER 5}

\section{A pilot study: Genetic variants may influence methotrexate plasma concentrations in pediatric malignancies}

Melanie M. Hagleitner

Sita H. Vermeulen

Peter M. Hoogerbrugge

Marieke J.H. Coenen

D. Maroeska W.M. te Loo 


\section{Abstract}

\section{Background}

High-dose methotrexate (MTX) forms a cornerstone in the treatment of several pediatric malignancies including acute lymphoblastic leukemia (ALL) and osteosarcoma. Delayed MTX elimination necessitates increased leucovorin rescue which may potentially annihilate the desired antitumor effect. We investigated if genetic variations in genes coding for enzymes in the MTX processing pathway are associated with delayed MTX elimination.

\section{Methods}

In total 92 children with ALL $(\mathrm{N}=49)$ and osteosarcoma $(\mathrm{N}=43)$ were genotyped for 40 functional genetic variants in 20 genes in the MTX processing pathway. MTX plasma concentrations at 48 hours following the start of each MTX course and MTX-induced toxicity (grades 0-4 according to the CTCAE criteria) were assessed. Differences in MTX levels between genotype groups were tested using generalized estimated equations (GEE) with robust standard errors to take repeated measurements into account. Analyses were performed under the assumption of an additive model.

\section{Results}

In total, 640 courses of high-dose MTX were evaluated. At least one episode of MTX-induced toxicity grade 3 or 4 was recorded in $41.8 \%$ of the patients. Four polymorphisms showed an association with MTX plasma levels with $p$-value <0.10: ABCC2 C.3972C $>\mathrm{T}$, AMPD1 C.34C>T, ITPA C.94C>T, GSTT1del. Association analyses for the four polymorphisms for MTX-induced toxicity (grade 3 and 4) showed however no statistically significant association.

\section{Conclusion}

This study identified four polymorphisms associated with elevated MTX plasma levels. Limited by small sample size and the retrospective nature of the study, the observed associations deserve further validation.

\section{Introduction}

High dose methotrexate (MTX; $1 \mathrm{~g} / \mathrm{m}^{2}-8 \mathrm{~g} / \mathrm{m}^{2}$ ) is widely used in the treatment of childhood cancer, including acute lymphoblastic leukemia (ALL) and osteosarcoma. Previous studies have shown that a systemic exposure to higher concentrations of MTX, including standard leucovorin rescue, is associated with an increased response to chemotherapy ${ }^{1-4}$. On the other hand, plasma concentrations greater than $1 \mu \mathrm{mol} / \mathrm{l}$ at 48 hours after MTX treatment are associated with increased risk of side effects ${ }^{5,6}$. Monitoring of MTX plasma levels allows early detection of a delayed elimination. Prolonged leucovorin rescue and/or hydration are administrated to prevent MTX toxicity when plasma levels at 48 hours after infusion are not $<0.4 \mu \mathrm{mol} / \mathrm{l}$. This strategy has significantly reduced the frequency of serious adverse effects and MTX-induced toxic deaths. However, prolonged leucovorin rescue should not be given too excessively as there may be a risk of potentially over-rescue these patients and annihilate the desired antitumor effect of MTX. For appropriate use of MTX it would therefore be useful to identify factors that affect MTX elimination and the risk of MTX-related adverse effects. Identifying patients with an a priori risk for high or low MTX clearance could be clinically useful to individualize MTX dosage and avoid toxic MTX exposure.

MTX is predominantly metabolized by the folate pathway. Studies in rheumatoid arthritis, where low-dose MTX is used as treatment, have suggested that polymorphisms in genes involved in the folate pathway may indeed influence the pharmacokinetics of MTX7-9. Until 2010 only a few predominantly single gene studies in ALL and no study in patients with osteosarcoma have been published regarding the role of pharmacogenetics on MTX plasma concentration. Genes studied were $G G H^{10}, A B C C 2^{11}$ and $M T H F R^{12,13}$. These studies indicate that variants in the folate pathway may explain interpatient variability in MTX plasma levels and/or MTX related toxicity. Here, we are the first to study candidate genes known to be involved in the MTX processing pathway regarding their influence on MTX levels at 48 hours after infusion and toxicity in ALL and osteosarcoma patients. 


\section{Patients and Methods}

\section{Patients and treatment}

In the period between 2002 and 2008, a total of 115 pediatric patients were subsequently treated with high-dose MTX at the Radboud university medical center. Their characteristics and clinical data were collected retrospectively from medical files. Clinical data concerning MTX levels were available in 92 children: 49 patients with de novo ALL and 43 primary, high-grade osteosarcoma patients. Complete data regarding MTX-induced toxicity grades were available in 90 children ( 42 with ALL, 48 with osteosarcoma). The study was approved by the local ethics committee and written informed consent was obtained from parents and/or patients. Patients with osteosarcoma were treated with 12 courses of high-dose MTX $\left(8-12 \mathrm{~g} / \mathrm{m}^{2}\right)$ according to the EURAMOS-1 protocol ${ }^{14}$. Patients with ALL were treated with four courses of high-dose MTX $\left(5 \mathrm{~g} / \mathrm{m}^{2}\right)$ according to the Dutch ALL-10 protocol ${ }^{15}$. In all patients, high-dose MTX was followed by leucovorin rescue treatment $\left(15 \mathrm{mg} / \mathrm{m}^{2}\right)$. Additional leucovorin rescue was performed if the MTX concentration was $\geq 0.4 \mu \mathrm{mol} / \mathrm{l}$ at 48 hours after administration. Interfering co-medication, such as trimethoprim and ciprofloxacin, was stopped $72 \mathrm{~h}$ before the start of MTX infusion.

\section{MTX levels and toxicity}

MTX concentrations were routinely determined by a fluorescence immunoassay. MTX plasma levels at 48 hours after the start of each MTX-infusion were used for the analyses. Toxicity grades after each high-dose MTX were registered in the period from start of the infusion until the next course of chemotherapy. We included leucopenia, neutropenia, trombopenia, anemia, epistaxis, mucositis, nausea, hepatotoxicity, nefrotoxicity, allergic reactions, and neurologic adverse events. Toxicity was graded according to the common terminology criteria for adverse events (CTCAE) of the National Cancer Institute (NCI) version $3.0^{16}$. For analyses, toxicity grades were dichotomized: grade 0,1 or 2 (no toxicity) versus grade 3 or 4 (toxicity).

\section{Selection of polymorphisms and genotyping}

Genomic DNA was isolated from blood using the QIAamp DNA Blood Midi kit (Qiagen, Venlo, The Netherlands) or saliva collected with the Oragene saliva collection kit (DNA Genotek, Kanata, ON, Canada) according to the manufacturer's protocols.

NCBI databases (PubMed, Gene) and the Pharmacogenomics Knowledge Base (www. pharmgkb.org) were searched for candidate genes known to be involved in the MTX processing pathway. In total, 15 of the most important genes reported to play a role in the folate cycle (TYMS, DHFR, CBS, MTHFR, SHMT, MTR, MTRR, MTHFD1, ATIC, AMPD1, GGH GST, FOLH1, ITPA and FPGS) were selected. Additionally, 5 genes coding for MTX transporters ( $A B C B 1, A B C C 1, A B C C 2, A B C G 2, S L C 19 A 1)$ were studied. In these 20 genes, polymorphisms that have been previously reported to be associated with MTX-pharmacokinetics and toxicity were selected for analysis. In total, 40 polymorphisms (Supplementary Table 1) were genotyped using Taqman allelic discrimination assays according to the protocol of the manufacturer (Invitrogen, Bleiswijk, The Netherlands). After amplification, the fluorescent signal for allelic discrimination was determined using the 7500 Fast Real-time System (Invitrogen). Automated allele calling was performed by allelic discrimination plots using SDS 1.4 software (Invitrogen). 


\section{Genetic association analysis}

Only polymorphisms with minor allele frequency of $>0.05$ and in Hardy-Weinberg equilibrium ( $p$-value $>0.05$ ) in the studied patient population were included in the analysis. The effect of the polymorphisms on MTX plasma concentration 48 hours after infusion was evaluated using a generalized estimating equation (GEE) analysis. This is an extended linear regression analysis in which correlations among repeated measurements obtained from individual subjects over time are taken into account. Regression coefficients and corresponding $95 \%$ confidence intervals (CI) were generated for each polymorphism under the assumption of an additive model (i.e. genotypes coded as 0 [homozygous most frequent genotype], 1 [heterozygous genotype] and 2 [homozygous least frequent genotype]; and included as continuous parameter); the resulting regression coefficient indicates the change in MTX levels associated with the minor allele. For the toxicity outcome, odds ratios (ORs) and $95 \%$ CIs were computed using logistic regression analysis, with the dependent variable being the highest grade of toxicity (dichotomized) according CTCAE criteria recorded throughout the treatment. Reported p-values are two-sided and are considered statistically significant if $<0.05$. Statistical analyses were conducted using Stata (version 11.2 ) and SPSS (version 16.0, SPSS, Chicago, IL, USA).

\section{Results}

Characteristics and treatment variables for the 92 children are reported in Table I. In total, 640 courses of high-dose MTX were evaluated, 193 courses in patients with ALL and 447 courses in patients with osteosarcoma. Elevated MTX plasma levels of $>0.4 \mu \mathrm{mol} / \mathrm{I}$ were seen after 173 MTX courses (27\%) of which $26(4 \%)$ had toxic values $>1 \mu \mathrm{mol} / \mathrm{l}$. MTX-induced toxicity grade 3 or 4 was recorded in 91 courses of which only 38 (41.8\%) were associated with a MTX plasma level of $>0.4 \mu \mathrm{mol} / \mathrm{l}$. Thus in 53 courses a grade 3 or 4 toxicity was seen with MTX plasma levels $<0.4 \mu \mathrm{mol} / \mathrm{l}$.

Table I. Characteristics and treatment variables for 640 courses of HD-MTX in 49 ALL and 43 osteosarcoma patients

\begin{tabular}{|lcc|}
\hline Age at diagnosis (in years) & Median & Range \\
ALL & & \\
Osteosarcoma & 5.9 & $1.2-13.6$ \\
Number of HD-MTX courses & 13.9 & $7.8-19.1$ \\
ALL & & \\
Osteosarcoma & 4 & $3-4$ \\
MTX Cumulative Dose $\left(\mathrm{g} / \mathrm{m}^{2}\right)$ & 10 & $7-12$ \\
ALL & & \\
Osteosarcoma & 16 & $12.8-20$ \\
MTX plasma level at 48 hours ( $\mu$ mol/l) & 117 & $21.1-144$ \\
ALL & & \\
Osteosarcoma & 0.35 & $0.25-0.38$ \\
$\quad$ & 0.21 & $0.16-0.34$ \\
Toxicity grade 3 or 4 & & \\
ALL & $\mathbf{N} /$ total & Percentage \\
Osteosarcoma & $20 / 48$ & $41.7 \%$ \\
& $16 / 42$ & $38.1 \%$ \\
\hline
\end{tabular}

Table II shows the results of the genetic association analyses. Three SNPs ( $A B C C 2$ c.1058G >A, ABCC1 c.3046-u1960G >A and GGH c.174 G>A) were excluded from analyses due to minor allele frequency (MAF) $<5 \%$; all SNPs were in Hardy-Weinberg equilibrium (HWE). None of the measured polymorphisms was associated to MTX plasma levels with $\mathrm{p}<0.05$. In total, four polymorphisms showed an association with MTX plasma levels with p-value <0.10: ABCC2 c.3972C > T, AMPD1 c.34C>T, ITPA c.94C>A, GSTT1 gene deletion. 


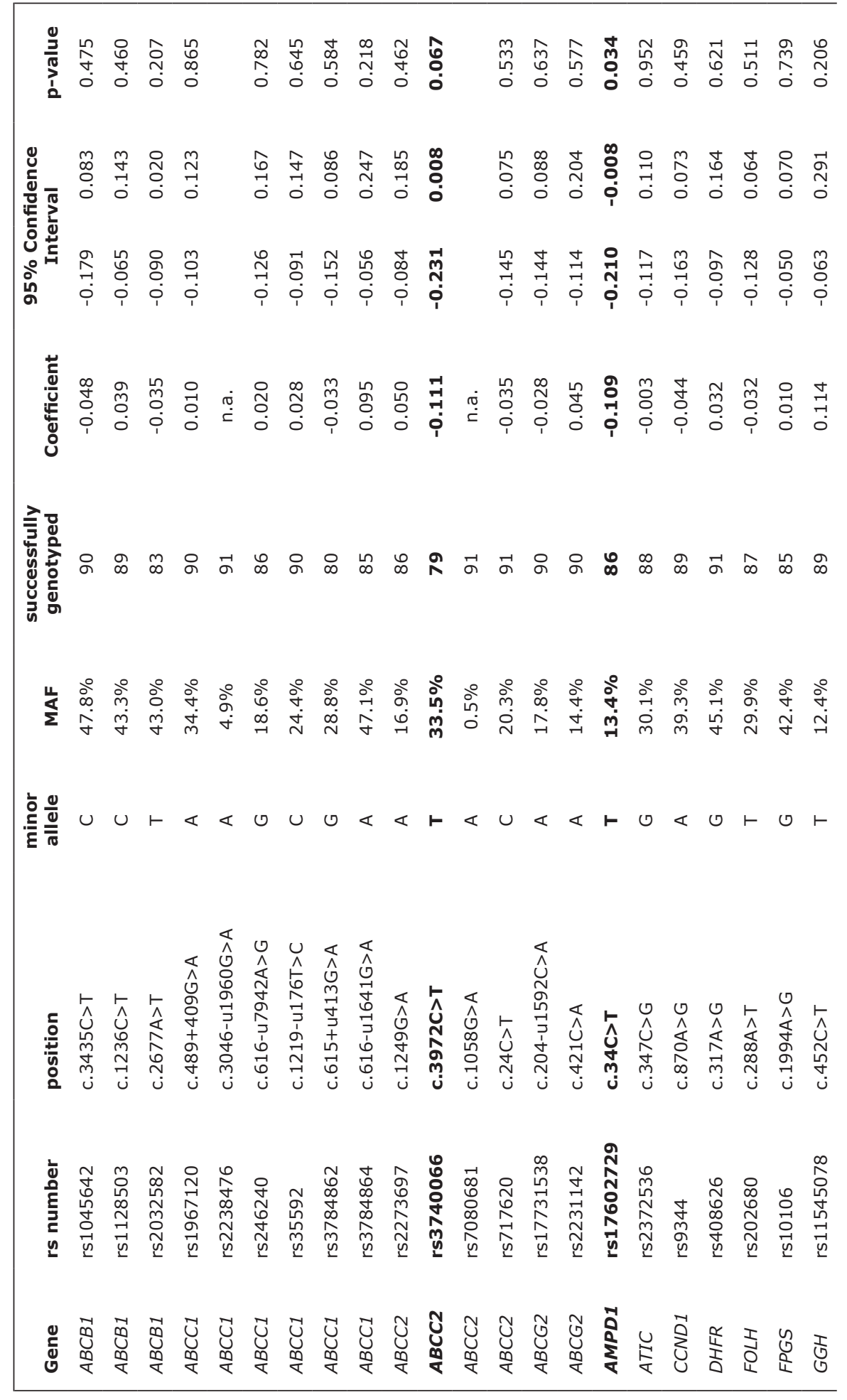

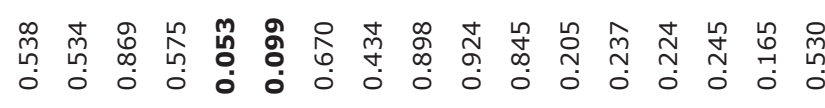

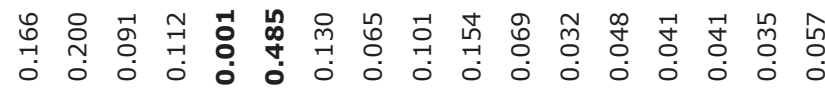

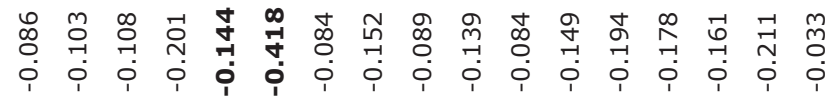

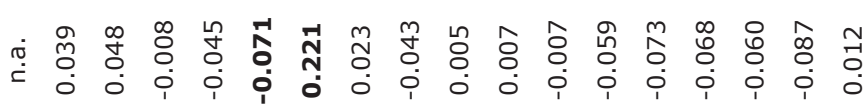

○ ロ

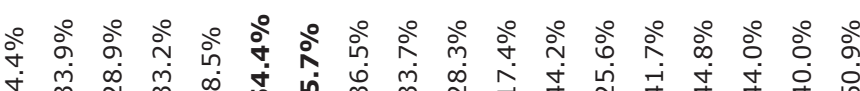

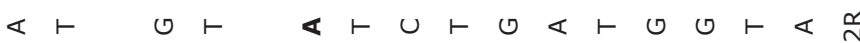

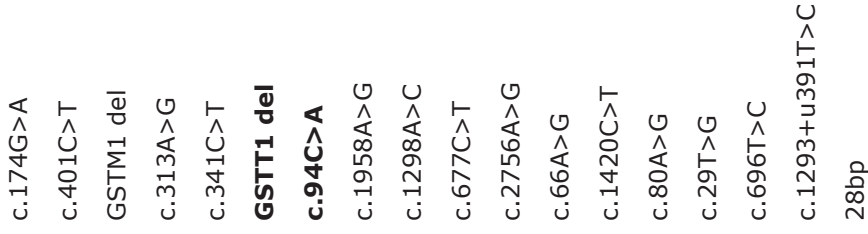

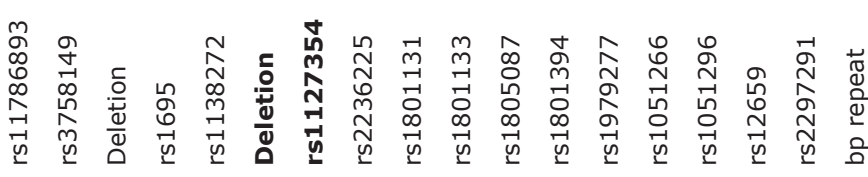

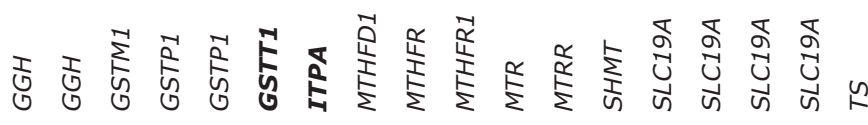

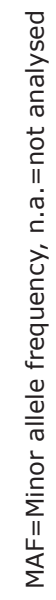


Table III shows the results for these polymorphisms in more detail. Indications for a non-additive model were observed for ITPA c.94C>A and the GSTT1 gene deletion. Notably, missing rates for $A B C C 2$ c.3972C $>$ T and the GSTT1 gene deletion were high (results for 15 and 13 patients missing, respectively).

Table III.

Genotypic regression coefficients for four SNPS associated with MTX plasma levels (with $p_{\text {additive }}<0.10$ )

\begin{tabular}{|cccccc|}
\hline SNP & Genotype & $\mathbf{N}$ & Coefficient & $\mathbf{9 5 \%}$ CI & p-value \\
\hline$A B C C 2$ & CC & 31 & - & - & - \\
$3972 C>T$ & CT & 40 & 0.11 & $0.063-0.291$ & 0.21 \\
& $\Pi$ & 6 & 0.22 & $0.059-0.379$ & 0.01 \\
\hline AMPD1 & CC & 63 & - & - & - \\
$34 C>T$ & CT & 21 & -0.11 & $-0.221-0.000$ & 0.06 \\
& $\Pi T$ & 1 & -0.23 & $-0.317-0.138$ & 0.00 \\
\hline ITPA & CC & 76 & - & - & - \\
$94 C>A$ & CA & 8 & 0.26 & $-0.079-0.605$ & 0.13 \\
& AA & 1 & 0.21 & $0.139-0.277$ & 0.00 \\
\hline GSTT1 & 0 copies & 15 & - & - & - \\
deletion & 1 copy & 42 & 0.04 & $-0.137-0.213$ & 0.67 \\
& 2 copies & 22 & -0.12 & $-0.260-0.02$ & 0.10 \\
\hline
\end{tabular}

Association analyses for the four polymorphisms regarding MTX-induced toxicity (grade 3 or 4 vs. 0,1 or 2 ) showed no statistically significant association (Table IV). Directionality of effect given the association results for MTX levels fitted expectations for $A B C C 2$ c.3972C $>$ T and AMPD1 c.34C >T, but not for ITPA c.94C >A and the GSTT1 gene deletion.
Table IV. Association with toxicity for four SNPS suggestively associated to MTX plasma levels

\begin{tabular}{|ccccccc|}
\hline \multicolumn{7}{c|}{ Patients with toxicity grade } \\
SNP & Genotype & $\mathbf{1}$ or $\mathbf{2}(\mathbf{N})$ & $\mathbf{3}$ or $\mathbf{4}(\mathbf{N})$ & OR & $\mathbf{9 5 \%} \mathbf{~ C I}$ & p-value \\
\hline ABCC2 & CC & 17 & 14 & 1.00 & - & - \\
$3972 C>T$ & CT & 27 & 13 & 2.40 & $0.22-1.54$ & 0.22 \\
& $T$ & 5 & 1 & 4.10 & $0.03-2.33$ & 0.27 \\
\hline AMPD1 & CC & 37 & 26 & 1.00 & - & - \\
$34 C>T$ & CT & 13 & 8 & 0.88 & $0.32-2.4$ & 0.79 \\
& $T$ & 1 & 0 & - & - & - \\
\hline ITPA & CC & 30 & 46 & 1.00 & - & - \\
$94 C>A$ & $C A$ & 4 & 4 & 1.53 & $0.36-6.60$ & 0.56 \\
& CA & 0 & 1 & - & - & - \\
\hline GSTT1 & 0 copies & 14 & 8 & 1.00 & - & - \\
deletion & 1 copy & 21 & 21 & 0.44 & $0.09-2.03$ & 0.29 \\
& 2 copies & 12 & 3 & 1.75 & $0.61-5.04$ & 0.30 \\
\hline
\end{tabular}




\section{Discussion}

Understanding individual variation in drug response and toxicity is one of the major challenges in cancer research today. While MTX is highly effective within the putative cytotoxic range, it is also associated with significant toxicity. In ALL and osteosarcoma, tools to identify patients at risk for toxic MTX plasma concentrations and MTX toxicity may allow treatment adaption by dose-adaption. The interpatient variability of the MTX metabolism makes it however hard to find the right balance between effectiveness and toxicity and it seems that monitoring MTX plasma levels alone is not enough as even patients with adequate MTX plasma levels can develop serious MTX toxicity. We are one of the first to study the association between germline variation in folate and MTXrelated genes in combination with toxicity in childhood cancer. This study was of exploratory nature but led to some interesting findings that warrant further replication.

Both $A B C C 2$ c.3972C $>$ T and AMPD1 c.34C $>$ T were suggestively associated with an increased elimination of MTX 48 hours after infusion and a lower risk to develop high grade toxicity. $A B C C 2$ codes for the multidrug resistance protein 2 (MRP2/ABCC2) which is the key actor for the biliary excretion of MTX. Decreased transport of MTX into the bile by $A B C C 2$ leads to an increased transport back into the circulation by $A B C C 3$ causing in creased MTX plasma concentrations ${ }^{17}$. However, functional studies on the effect of the genetic variant in $A B C C 2$ have not been published. In contrast to our data, the c.3972 T allele was significantly associated with increased MTX levels at 48 hours in the study of Rau and colleagues in 44 patients with ALL $^{11}$. In a pharmacokinetic study where MTX elimination and distribution was studied via a multi-compartment model in $45 \mathrm{ALL}$ patients, this polymorphism had however no significant influence on MTX pharmacokinetic parameters ${ }^{18}$. The AMPD1 gene encodes the enzyme adenosine monophosphate deaminase, which plays an important role in the purine nucleotide cycle. The c.34C>T variant causes a premature stop in the protein, leading to a nonfunctional AMPD1 enzyme. In contrast to our study, in patients with rheumatoid arthritis the presence of this variant showed no influence on the MTX plasma concentration but an association with central nervous system adverse effects ${ }^{19}$. There are no published data on the influence of AMPD1 c.34C $>$ T regarding highdose MTX plasma levels or MTX-induced toxicity in patients with cancer.

We also found indications for a role of the ITPA c.94C >A variant and GSTT1 copy number on MTX plasma levels. However, their effect on toxicity was not in line with the plasma level results. Patients with a complete deletion of GSTT1 showed lower MTX plasma levels at 48 hours after infusion but had an increased risk to develop MTX-related toxicity. In contrast, the ITPA c.94C >A variant was associated with higher MTX plasma levels at 48 hours after infusion but no effect on the development of MTX-induced toxicity was seen In general, glutathione S-transferases (GSTs) are linked to the development of cytotoxicity of chemotherapeutic drugs, including alkylating agents such as MTX20. It might be expected that patients with a complete deletion of GSTT1 have a reduced metabolism of MTX leading to elevated plasma levels and increased toxicity which is in contrast to the data of this study. The ITPA gene encodes for an inosine triphosphate pyrophosphatase. It has been shown that the ITPA c.94C $>T$ polymorphism causes an amino-acid change (P32T), reducing ITPA enzymatic activity to $25 \%$ in heterozygotes, and abolishing it in homozygous variant ${ }^{21}$. Individuals with an homozygous variant might have less ability to metabolize MTX and therefore higher plasma levels which is in line with our findings. Furthermore, this variant has been found to influence the efficacy of MTX treatment of rheumatoid arthritis ${ }^{22}$ and $\mathrm{ALL}^{23}$ which could not be confirmed with our data.

In this study, we focused on candidate genes and polymorphisms that are likely to have a direct impact on the function of the gene as to increase our chance to identify relevant variants. We optimized our power by using data on available repeated measures of MTX plasma levels and by combining the results for osteosarcoma and ALL patients. Obvious pitfalls include the lack of power due to small sample size, potentia inadequate pre-selection of candidate genes, unexplored heterogeneity in SNP effects for ALL and osteosarcoma patients. Nevertheless, our results can provide an incentive for future studies into pharmacogenetics of MTX in childhood cancer. These studies would ideally include pharmacokinetic parameters and functional validation. 


\section{References}

Ferrari S, Sassoli V, Orlandi M, et al. Serum methotrexate (MTX) concentrations and prognosis in
patients with 1993;5:135-141. Relling MV, Fairclough D, Ayers D, et al. Patient characteristics associated with high-risk methotrexate con-
centrations and toxicity. J Clin Oncol. 1994;12:1667-1672. Delepine N, Delepine $G$, Bacci $G$, et al. Influence of methotrexate dose intensity on outcome of patients with
high grade osteogenic osteosarcoma. Analysis of the literature. Cancer. 1996; $78: 2127-2135$.

Mikkelsen TS, Sparreboom A, Cheng C, et al. Shortening infusion time for high-dose methotrexate alters

Isacoff WH, Morrison PF, Aroesty ], et al. Pharmacokinetics of high-dose methotrexate with citrovorum factor

$6 \begin{aligned} & \text { Stoller } R G \text {, Hande } K R \text {, Jacobs } S A \text {, et al. Use of plasma pharmacokinetics to predict and prevent methotrexate } \\ & \text { toxicity. N Engl J Med. 1977;297:630-634. }\end{aligned}$

7 Wessels JAM, Kooloos WM, de Jonge R, et al. Relationship between genetic variants in the adenosine pathway

Van Ede AE, Laan RF, Blom HJ, et al. The C677T mutation in the methylenetetrahydrofolate reductase gene: a genetic risk factor for methotrexate Arthritis Rheum. $2001 ; 44: 2525-2530$.
Ant

9 Dervieux $\mathrm{T}$, Greenstein $\mathrm{N}$, Kremer J. Pharmacogenomic and metabolic biomarkers in the folate pathway and

10 Cheng Q, Wu B, Kager L, Panetta JC, et al. A substrate specific functional polymorphism of human [gammaj-glutamyl hydrolase alters catalytic activity and methotrexate polyg
lymphoblastic leukaemia cells. Pharmacogenet Genomics. 2004;14:557.

11 Rau T, Erney B, Göres R, et al. High-dose methotrexate in pediatric acute lymphoblastic leukemia: Impact of
ABCC2 polymorphisms on plasma concentrations. Clin Pharmacol Ther. 2006:80:468-476.

Shimasaki N, Mori T, Samejima $\mathrm{H}$, et al. Effects of methylenetetrahydrofolate reductase and reduced folate carrier 1 polymorphisms on high-dose methotrexate-induced toxi
leukemia or lymphoma. J Pediatr Hematol Oncol 2006;28:64-68.

13 Kantar M, Kosova B, Cetingul N, et al. Methylenetetrahydrofolate reductase C677T and A1298C gene poly morphisms and therapy-related toxicitity in children treated for acute lymphoblastic leukemia and non-Hod-

14 Whelan J, Seddon B, Perisoglou M. Management of osteosarcoma. Curr Treat Options Oncol 2006; 7:444-455

15 Kamps WA, van der Pal-de Bruin KM, Veerman AJ, et al. Long-term results of Dutch Childhood Oncology
Group studies for children with acute lymphoblastic leukemia from 1984 to 2004. Leukemia 2010;24:309319 .

16 Common toxicity criteria version 3.0 (National Cancer Institute Website). Available at: http://ctep.info.nih.

17 Vlaming MLH, Pala Z, van Esch A, et al. Impact of ABCC2 and ABCC3 on the in vivo elimination of methotrex-

18 Simon N, Marsot A, Villard E, et al. Impact of ABCC2 polymorphisms on high-dose methotrexate pharmacoki-

19 Stamp LK, Chapman PT, O'Donnell IL, et al. Polymorphisms within the folate pathway predict folate concentrations but are not associated with dis
Pharmacogenet Genomics. 2010;20:367-376.

20 Tsuchida S, Sato K. Glutathione transferases and cancer. Crit Rev Biochem Mol Biol. 1992;27:337-384.

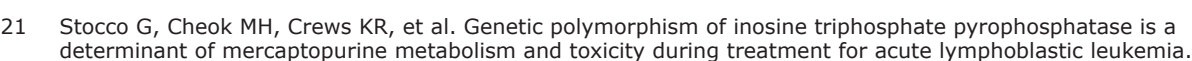
determinant of mercaptopurine metabol
Clin Pharmacol Ther. 2009;85:164-172.
22 Dervieux $\mathrm{T}$, Wessels JA, Van der ST, et al. Gene-gene interactions in folate and adenosine biosynthesis pathways affect me
2009;19:935-944.

$23 \mathrm{Kim} \mathrm{H}$, Kang HJ, Kim HJ, et al. Pharmacogenetic analysis of pediatric patients with acute lymphoblastic leu- 

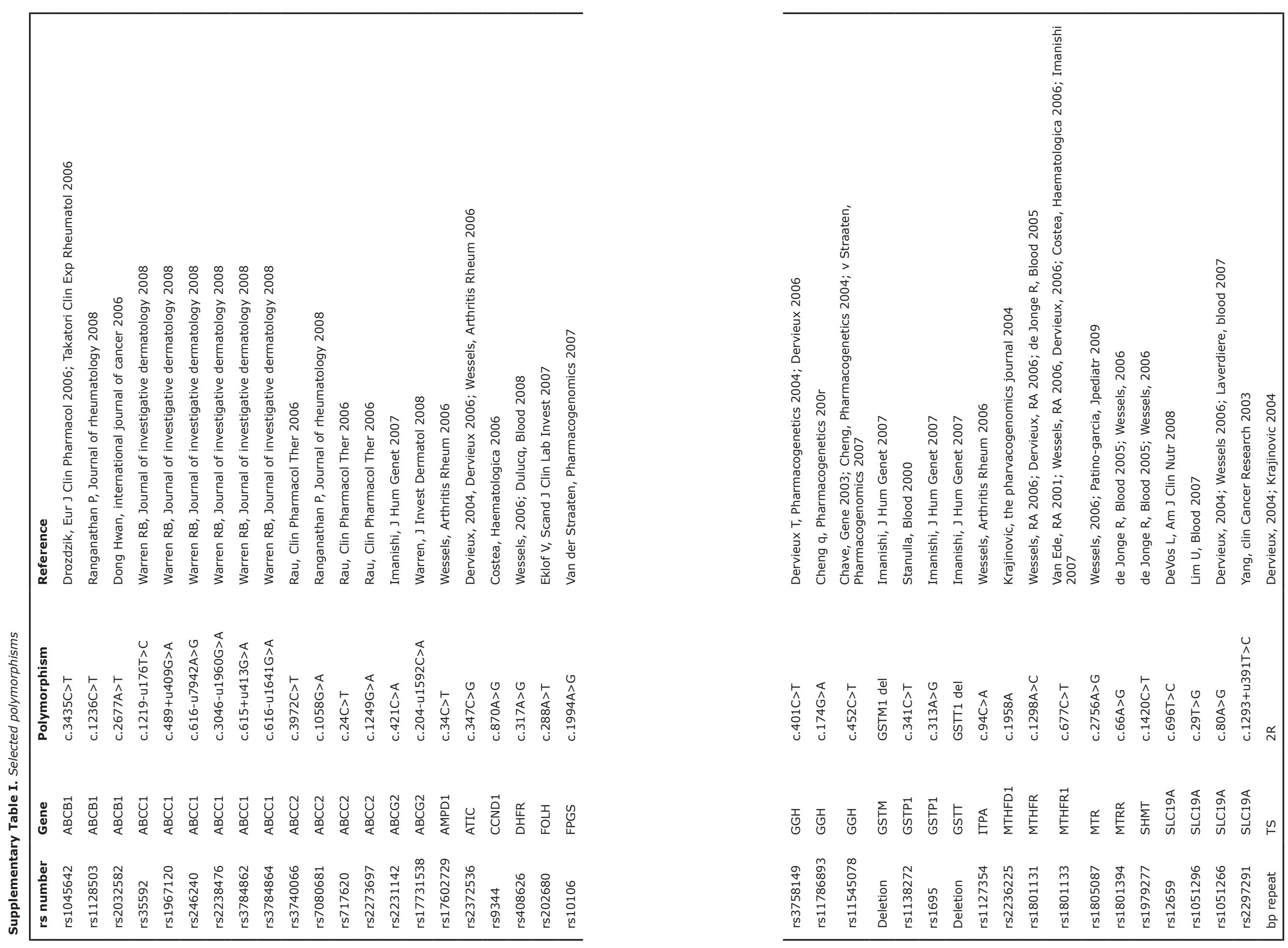

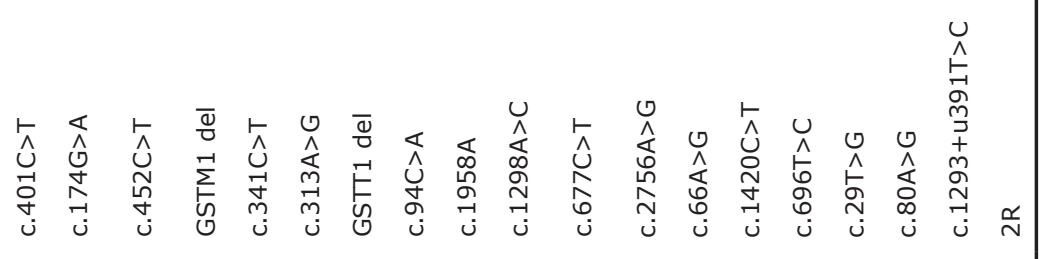

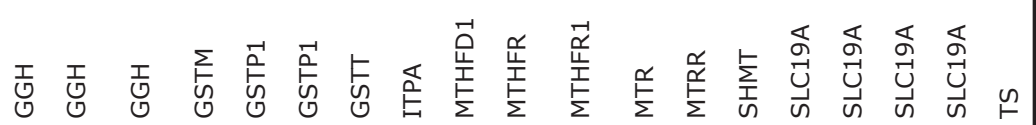

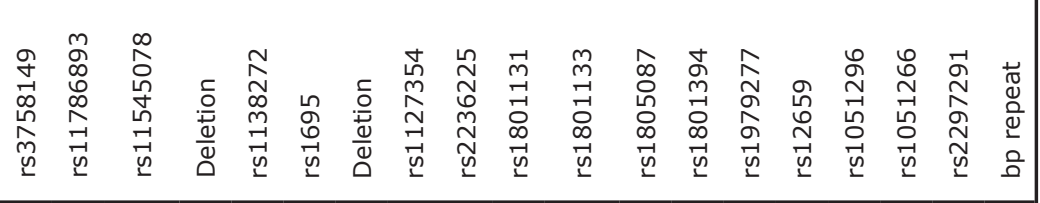





\section{CHAPTER 6}

\section{The role of the}

MTHFR 677C $>$ T polymorphism in methotrexate-induced liver toxicity: a meta-analysis in patients with cancer

Melanie M. Hagleitner

Marieke J.H. Coenen

Richard Aplenc

Ana Patiño-Garcia

Patrizia Chiusolo

Donato Gemmati
Monica De Mattei

Alessia Ongaro

Maja Krajinovic

Peter M. Hoogerbrugge

Sita $\mathrm{H}$. Vermeulen

D. Maroeska W.M. te Loo 


\section{Abstract}

Methotrexate (MTX), one of the important pillars in the treatment of different forms of cancer, is associated with the development of hepatotoxicity. The 677C >T variant ( $r s 1801133$ ) in the methylenetetrahydrofolate reductase (MTHFR) gene might affect the development of hepatotoxicity. Results in literature are, however, contradictive. The aim of this study was to evaluate the role of the MTHFR $677 \mathrm{C}>$ T polymorphism in MTX-induced hepatotoxicity by analyzing a Dutch cohort of pediatric patients treated with high doses of MTX and subsequently performing a meta-analysis.

Ninety-eight patients receiving 542 courses of high-dose MTX were genotyped for the MTHFR $677 \mathrm{C}>\mathrm{T}$ variant. Hepatotoxicity was evaluated retrospectively according to CTCAE-NCI criteria. The influence of MTHFR $677 \mathrm{C}>\mathrm{T}$ on hepatotoxicity was examined using a generalized estimating equation analysis (GEE). A fixed-effect meta-analysis based on this and previous studies investigating the association between the MTHFR $677 \mathrm{C}>$ T polymorphism and uniformly coded hepatotoxicity was performed. The GEE analysis showed an increased risk of developing hepatotoxicity for T- versus C-allele (odds ratio (OR) $1.8 ; 95 \%$ confidence interval (CI) $1.0-3.2, p=0.04$ ). This finding was not supported by the meta-analysis including seven studies and 1044 patients; the OR for the $677 \mathrm{~T}$ versus $\mathrm{C}$ allele was 1.1 ( $95 \% \mathrm{CI} 0.84-1.5, \mathrm{p}=0.25)$. Heterogeneity between studies was observed, possibly related to differences in MTX dose and leucovorin rescue. In conclusion, in patients with cancer the MTHFR 677T allele has only a minor role in the development of MTX induced hepatotoxicity. Observed heterogeneity between studies warrants further study into (tailored) leucovorin rescue.

\section{Introduction}

Hepatotoxicity is one of the most common side effects of methotrexate (MTX) and occurs in $15-50 \%$ of patients but is usually transient and rarely results in chronic liver disease ${ }^{1}$ However, if the levels of the liver enzyme Alanine Transferase (ALT) have not normalized before the start of the next treatment course, chemotherapy generally is postponed and/or reduced. Reduction of chemotherapy might increase the risk of recurrence of the disease ${ }^{2}$ Predicting which patients are at risk for MTX-induced hepatotoxicity is not possible due to the unexplained inter-patient variability in pharmacokinetics and pharmacodynamics of MTX. Several studies have suggested that pharmacogenetics may have an additional value to explain this inter-patient variability and might be useful to identify patients at risk. A large number of studies have shown that the clinical variation in response to MTX is associated with polymorphisms in genes involved in MTX degradation and processing ${ }^{3-5}$. The most widely studied single nucleotide polymorphism (SNP) with respect to MTX metabolism is the $677 \mathrm{C}>\mathrm{T}$ variant in the methylenetetrahydrofolate reductase (MTHFR) gene. MTHFR is a key enzyme for intracellular folate homeostasis and metabolism. The T allele of the genetic variant is associated with decreased activity of the enzyme MTHFR resulting in low folate levels ${ }^{6}$. Based on these data, it has been suggested that patients with reduced MTHFR enzyme activity are more susceptible to toxicities from MTX. Several studies have indeed described an increased risk of toxicity ${ }^{7,8}$. However, not all studies have been able to link presence of the $677 \mathrm{~T}$ allele to an increased risk of MTX-induced toxicity ${ }^{9-11}$.

As data published regarding the role of the MTHFR 677 polymorphism are contradictive, we analyzed the association between the MTHFR 677C >T SNP and hepatotoxicity in a Dutch cohort of cancer patients treated with high dose MTX. In addition, a meta-analysis including a total of 1044 patients was performed and potential causes of the heterogeneous results between different studies were explored. 


\section{Patients and Methods}

\section{Patients and treatment}

The patient population in this study comprised a total of 115 Dutch pediatric oncology patients treated at Radboud University Medical Center from November 2001 until January 2008. These include 63 newly diagnosed, high-grade osteosarcoma patients and 52 patients with de novo acute lymphoblastic leukemia (ALL). The study was approved by the local ethics committee of the Radboud University Medical Center and written informed consent was obtained from parents and/or patients. Patients with osteosarcoma were treated with 12 courses of high-dose MTX $\left(8-12 \mathrm{~g} / \mathrm{m}^{2}\right)$ according to the EURAMOS-1 protocol ${ }^{12}$ Patients with ALL were treated with 4 courses of high-dose MTX $\left(5 \mathrm{~g} / \mathrm{m}^{2}\right)$ according to the Dutch ALL-10 protocol ${ }^{13}$. In all patients high-dose MTX was followed by leucovorin rescue treatment $\left(15 \mathrm{mg} / \mathrm{m}^{2}\right)$. If the MTX concentration was higher than $0.4 \mu \mathrm{mol} / \mathrm{L}$ at 48 hours after administration, additional leucovorin rescue was performed. Interfering comedication, such as trimethoprim and ciprofloxacin, was stopped 72 hours before the start of MTX infusion. The patient characteristics and clinical data were collected retrospectively from medical files.

\section{Toxicity}

ALT activity was used to assess hepatotoxicity. Peripheral blood samples for measurement of ALT were obtained with each chemotherapy course at 48 hours after the start of the MTX infusion. Toxicity was graded according to the common terminology criteria for adverse events (CTCAE) of the National Cancer Institute (NCI) version 3.014. For analyses, toxicity grades were dichotomized: grade 0,1 or 2 versus grade 3 or 4 .

\section{Genotype analysis}

DNA was extracted from blood using the QIAamp DNA Blood Midi kit (Qiagen Inc., Valencia, CA. USA) according to the manufacturers' protocol. DNA of saliva (2 $\mathrm{ml})$ collected in the Oragene saliva collection kit (DNA Genotek, Kanata, Ontario, Canada) was isolated using the Oragene DNA purification protocol as supplied by the manufacturer The polymorphism 677C>T ( $r$ 1801133) in MTHFR was genotyped using Taqman ${ }^{\circledR}$ allelic discrimination assays according to the protocol of the manufacturer (Applied Biosystems, Nieuwerkerk aan den Ijssel, The Netherlands (assay ID C 1202883 20). After amplification the fluorescent signal for allelic discrimination was determined using the 7500 Fast Real-time System (Applied Biosystems). Automated allele calling was performed by allelic discrimination plots using SDS 1.4 software (Applied Biossystems).

\section{SNP analysis}

The effect of the MTHFR polymorphism on the dichotomized toxicity variable was evaluated using a generalized estimating equation analysis (GEE), an extended regression analysis in which correlations among repeated measurements obtained from individual subjects over time were taken into account. Reported p-values are two-sided and are considered statistically significant if $<0.05$. Statistical analyses were conducted using Stata (version 9.2) and SPSS (version 16.0, SPSS Inc, Chicago, II).

\section{Meta-analysis}

We searched PubMed for papers before December 2010 using the keywords: 'MTHFR C677T', 'cancer' and 'toxicity or hepatotoxicity' (Figure I). Thirty-two articles were identified. References listed in the identified articles and reviews were used to find additional studies. All articles were screened for relevance to MTX-induced hepatotoxicity in oncology patients. Meta-analysis was performed on those studies evaluating the association of the MTHFR $677 \mathrm{C}>$ T polymorphism and MTX in Caucasian adults or pediatric patients with cancer. 
Figure I. Flow chart showing paper selection and exclusion for the meta-analysis

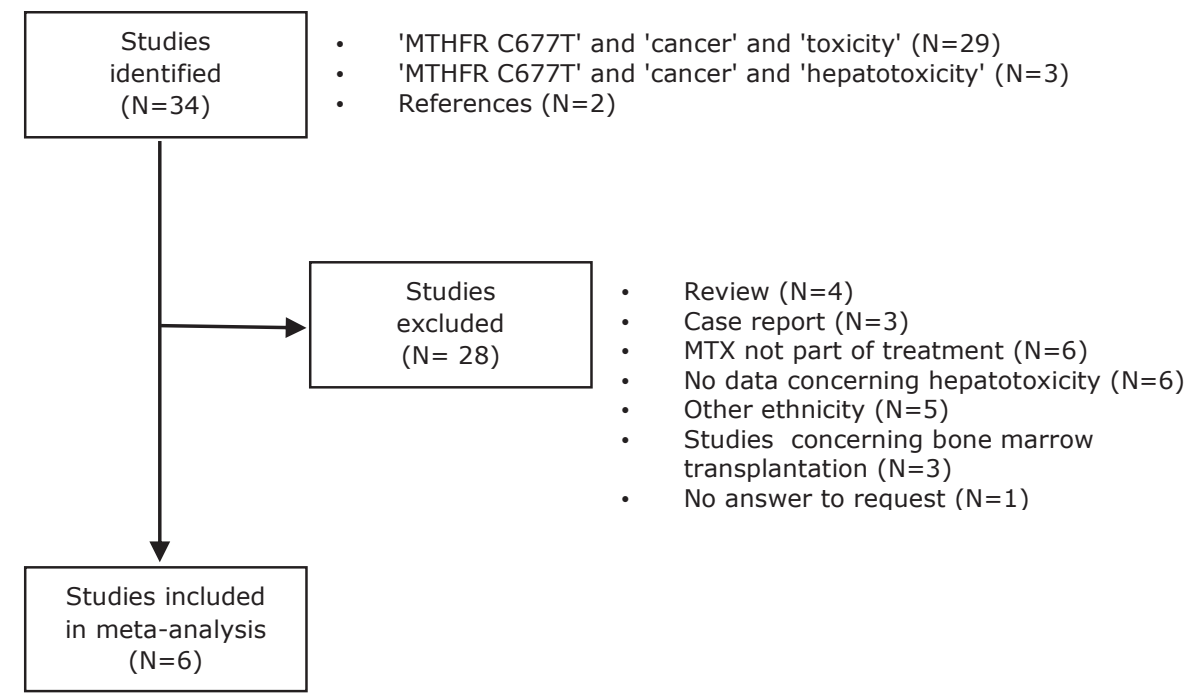

Because of different definitions of hepatotoxicity, corresponding authors of these studies were contacted for data on genotype frequencies in patients with and without hepatotoxicity according to the dichotomized CTCAE-NCI toxicity grades. The highest grade of CTCAE toxicity observed in each patient during the therapy period was recorded. Allelic odds ratio (OR) and $95 \%$ confidence interval (CI) were estimated using a fixed-effects model. The studies were weighed using the inverse variance method where larger studies with smaller standard errors have more weight than smaller studies with larger errors (Review manager 5.0, The Cochrane Collaboration, Oxford, UK). In addition, subgroup analyses were conducted by age and MTX dosage. Heterogeneity among studies was examined with $\mathrm{I}^{2}$ statistics that can be interpreted as the proportion of total variation contributed by between-study variation.

\section{Results}

\section{Patients}

Clinical data concerning MTX-induced hepatotoxicity and adequate samples for genotyping were available for 49 patients with osteosarcoma and 49 patients with ALL. The patient characteristics are reported in Table I. In total, 542 courses of high-dose MTX were evaluated. Hepatotoxicity grades 3 or 4 according to the NCI criteria were seen in 160 courses of 37 different patients, predominantly among patients with osteosarcoma (34 of 49 patients with osteosarcoma vs. 3 of 49 patients with ALL, $p=0.001$ ). The median time to occurrence of severe hepatotoxicity was after the third course of MTX (range $1^{\text {st }} 7^{\text {th }}$ course).

\section{SNP association analysis}

The MTHFR $677 \mathrm{C}>\mathrm{T}$ allele and genotype frequencies were consistent with previous published reports ${ }^{15}$ and were in Hardy-Weinberg equilibrium. Thirty-eight patients (38.7\%) carried a heterozygous genotype (CT), 8 patients $(8.2 \%)$ were homozygous $\Pi$, and 52 patients $(53.1 \%)$ carried the CC genotype. Genotype frequencies were similar but not the same in the ALL and osteosarcoma groups (Table I).

\begin{tabular}{|c|c|c|}
\hline & ALL & Osteosarcoma \\
\hline \multicolumn{3}{|c|}{ Age at diagnosis (years) } \\
\hline median (range) & $5.9(2.1-13.6)$ & $13.9(7.8-19.0)$ \\
\hline \multicolumn{3}{|c|}{ MTX cumulative dose $\left(\mathrm{g} / \mathrm{m}^{2}\right)$} \\
\hline median (range) & $16(5-20)$ & $117(30-144)$ \\
\hline \multicolumn{3}{|l|}{ MTX courses } \\
\hline median (range) & $4(1-4)$ & $7(2-12)$ \\
\hline \multicolumn{3}{|c|}{ Hepatotoxicity grade 3-4 } \\
\hline number $(\%)$ & $3(6 \%)$ & $34(69 \%)$ \\
\hline \multicolumn{3}{|l|}{ MTHFR $677 \mathrm{C}>\mathrm{T}$} \\
\hline $\mathrm{CC} n(\%)$ & $28(57.1 \%)$ & $24(48.9 \%)$ \\
\hline CT n (\%) & $18(36.7 \%)$ & $20(40.8 \%)$ \\
\hline$\pi \mathrm{n}(\%)$ & $3(6.1 \%)$ & $5(10.2 \%)$ \\
\hline
\end{tabular}


It is reasonable to expect that the MTHFR $677 \mathrm{C}>\mathrm{T}$ polymorphism has a different effect on different types of cancer. Therefore, the type of cancer was taken in consideration in the GEE-analysis. The GEE analysis on the effect of the MTHFR polymorphism on the dichotomized toxicity variable after adjustment for type of cancer (i.e. osteosarcoma or ALL) showed a higher risk of developing hepatotoxicity in the CT (OR=1.8; 95\% CI: $0.76-4.2$ $\mathrm{p}=0.19)$ and $\Pi(\mathrm{OR}=3.3 ; 95 \% \mathrm{CI}: 0.97-11.0, \mathrm{p}=0.06)$ patients compared to the reference CC group, though not statistically significant. The analysis under an additive model showed a 18 -fold increased risk was predominantly seen in patients with osteosarcoma where the T-allele was associated with a 1.9-fold increased risk of developing hepatotoxicity (95\% CI: 1.0-3.5, $\mathrm{p}=0.050$ ) in the ALL group we found a non-statistically significant 1.3 -fold increased risk ( $95 \% \mathrm{CI}$ : $0.28-6.4, p=0.72$ ) of developing hepatotoxicity for the $T$ versus $C$ allele.

\section{Meta-analysis}

Of the 32 studies identified in the literature, 7 discussed the $677 \mathrm{C}>\mathrm{T}$ polymorphism in relation to MTX-induced hepatotoxicity in a Caucasian population (Figure I). The corresponding authors of 6 studies were willing to reclassify their data and stratify according to the NCI criteria as described above $4,7,9,16-18$. However, this information was only available in a subset $(\mathrm{N}=1044)$ of all patients $(\mathrm{N}=1394)$ included in these seven studies including our own study. Table II shows the conclusions of the initial studies as well as the number of patients per study that were included in the meta-analysis. Included studies varied in age, type of cancer, MTX doses and frequency of hepatotoxicity.

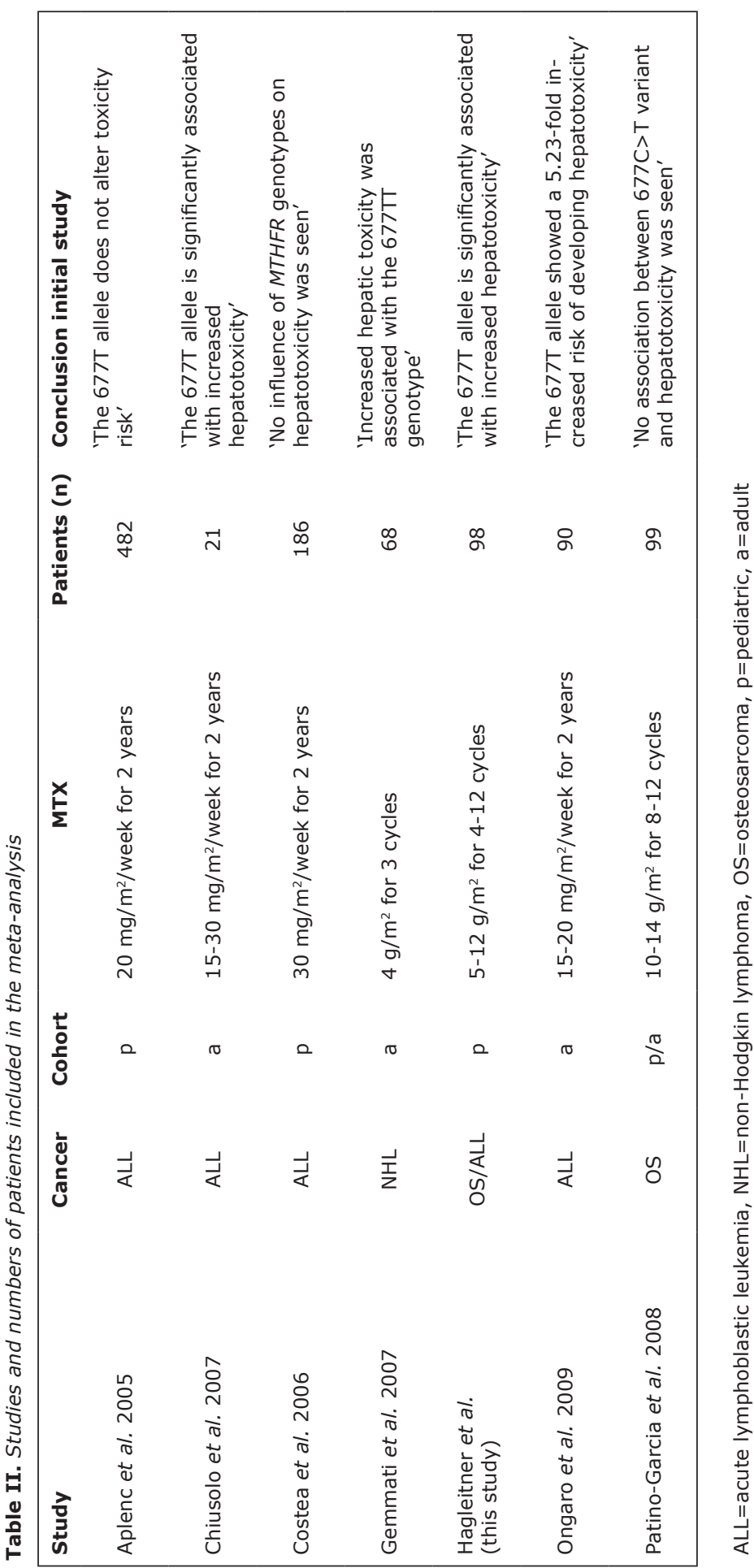


Based on the reclassified toxicity data, allelic ORs were recalculated for each individual study (Figure II). When assessed in combination with the results of this study, no significant association between the $677 \mathrm{C}>\mathrm{T}$ variant and hepatotoxicity was seen (OR T vs. C allele $=1.1,95 \% \mathrm{CI}: 0.84-1.5, \mathrm{p}=0.25$ ). The heterogeneity test showed a non-statistically significant heterogeneity with an $\mathrm{I}^{2}$ of $24 \%$.

Figure II.

Meta-analysis of the association between the MTHFR 677C>T variant and the risk for hepatotoxicity

\begin{tabular}{|c|c|c|c|c|c|c|c|}
\hline Study or Subgroup & $\log [\mathrm{OR}]$ & SE & $\begin{array}{l}\text { Hepato } \\
\text { G 3-4 }\end{array}$ & $\begin{array}{l}\text { toxicity } \\
\text { G 0-2 }\end{array}$ & Weight & $\begin{array}{l}\text { Odds Ratio } \\
\text { IV, Random, } 95 \% \text { CI }\end{array}$ & $\begin{array}{c}\text { Odds Ratio } \\
\text { IV, Random, } 95 \% \mathrm{CI}\end{array}$ \\
\hline Aplenc, 2005 & 0 & 0.14 & 362 & 602 & $39.4 \%$ & $1.00[0.76,1.32]$ & 7 \\
\hline Chiusolo, 2007 & 1.992 & 1.1114 & 12 & 30 & $1.6 \%$ & $7.33[0.83,64.74]$ & \\
\hline Costea, 2007 & -0.0305 & 0.2284 & 240 & 132 & $24.2 \%$ & $0.97[0.62,1.52]$ & - \\
\hline Gemmati, 2007 & 0.3646 & 1.4146 & 2 & 134 & $1.0 \%$ & $1.44[0.09,23.04]$ & \\
\hline Hagleitner, this study & 0.5988 & 0.2876 & 74 & 122 & $17.7 \%$ & $1.82[1.04,3.20]$ & $\because$ \\
\hline Ongaro, 2009 & 0.1989 & 0.6087 & 12 & 168 & $5.0 \%$ & $1.22[0.37,4.02]$ & \\
\hline Patino-Garcia, 2009 & & 0.3899 & & 162 & $11.0 \%$ & & \\
\hline & & & & & & $1.12[0.84,1.48]$ & \\
\hline
\end{tabular}

Additionally, we performed a meta-analysis stratified for gender (males $\mathrm{OR}=1.06,95 \% \mathrm{CI}$ : $0.73-1.53, p=0.76$ and females $O R=1.71,95 \%$ CI: $1.04-2.81, p=0.03$ ).

Next we also stratified the cohorts based on MTX dosage (MTX $15-30 \mathrm{mg} / \mathrm{m}^{2} /$ course and MTX $4-14 \mathrm{~g} / \mathrm{m}^{2} /$ course). In both groups the MTHFR $677 \mathrm{C}>\mathrm{T}$ variant did not affect hepatotoxicity risk $\left(15-30 \mathrm{mg} / \mathrm{m}^{2} /\right.$ course, $\mathrm{N}=932, \mathrm{OR}=1.34,95 \% \mathrm{CI}: 0.83-1.3, \mathrm{p}=0.75$; and 4-14 $\mathrm{g} / \mathrm{m}^{2}$ (course, $\mathrm{N}=462, \mathrm{OR}=1.04,95 \% \mathrm{CI}: 0.83-1.30, \mathrm{p}=0.75$ ). Furthermore, we assessed differences in the association with hepatic toxicity between pediatric and adult patients. This analysis showed that the MTHFR $677 \mathrm{C}>\mathrm{T}$ variant was not associated with hepatic toxicity in pediatric patients $(\mathrm{N}=734 ; \mathrm{OR}=0.99$ ( $95 \% \mathrm{CI}: 0.79-1.25, \mathrm{p}=0.95)$. In adult patients, a non-statistically significant increased risk was observed $(\mathrm{N}=198 ; \mathrm{OR}=2.14$ (95\% CI: 0.82-5.61, $\mathrm{p}=0.12$ ).

\section{Discussion}

In the present study, we investigated whether the MTHFR $677 \mathrm{C}>\mathrm{T}$ genotype was associated with MTX-induced hepatotoxicity in pediatric patients with cancer. Analyses of our data on 98 patients showed analogue to published data indecisive results. Patients carrying the 677TT-genotype showed a clear trend, although statistically not significant, increased risk to develop hepatotoxicity. Furthermore, this effect was mainly seen in the group of patients with osteosarcoma who were treated with very high doses of MTX (cumulative dose range: $30-144 \mathrm{~g} / \mathrm{m}^{2}$ ) compared to ALL patients (cumulative dose range: $5-20 \mathrm{~g} / \mathrm{m}^{2}$ ). A possible explanation for the more modest effect observed in the ALL patients could be that in patients with a lower dose of MTX the leucovorin rescue of nonmalignant cells might be efficient enough to compensate the negative effect of reduced MTHFR activity. It has been shown that adequate folate levels can reduce or even normalize the effects related to the MTHFR polymorphism ${ }^{19}$. Additional folate supplementation after higher doses of MTX may therefore counteract the effect of a reduced MTHFR activity. Thus the combination of higher doses of MTX and insufficient rescue of non-malignant cells may lead to a higher risk of developing hepatotoxicity in patients carrying the 677T-genotype. This explanation is supported by a recent study by Chiusolo et al. suggesting that intensive leucovorin rescue after high-dose MTX abrogates the effect of the MTHFR 677C $>$ T variant ${ }^{20}$.

The second part of our study concerned a meta-analysis of 6 previously published studies and our own study. Overall, the meta-analysis did not show an association between the MTHFR $677 \mathrm{C}>$ T variant and low $(0,1$ and 2$)$ and high-grade $(3,4)$ MTX-induced hepatotoxicity (OR $1.1,95 \% \mathrm{CI}: 0.84-1.5)$. We did not find statistically significant heterogeneity between study results but inspection of the effect estimates showed large differences in point estimates, despite of uniform reclassification of toxicity grading. Several factors, including differences in patient characteristics and/or treatment protocols, may be the cause of this difference. Results of our meta-analyses were stratified for age showing no association between age and development of hepatotoxicity. Another difference might be that the studies in the meta-analysis included patients with three different types of cancer. However, it is reasonable to expect the effect of a germline genetic variant in a drug metabolizing gene to be independent of the type of malignancy. Indeed, the MTHFR 677C >T variant has been associated with decreased activity of MTHFR and prolonged low folate state in affected patients in different kind of diseases ${ }^{21,22}$. A patient was either classified as positive or negative for hepatotoxicity based on the highest CTCEA grade experienced during the treatment period. Although this reclassification resulted in homogeneous toxicity grading over studies, it does not fully capture the experienced toxicity per patient. This inaccuracy in phenotype classification may have also contributed to heterogeneity between study estimates. Gender has recently been pointed out by several authors as an important factor interacting with MTHFR polymorphism in producing a phenotypic effect on 
the patients from cancer susceptibility ${ }^{23}$ to migraine ${ }^{24}$. Our subgroup analyses by gender indeed showed a significant association between the MTHFR T-allele and hepatotoxicity for females. However, our results also show a wide $95 \%$ CI which might be explained by less females (38.8\%) included in the studies compared to males (61.2\%). As far as we know this is the first meta-analysis showing a significant interaction between the MTHFR genotype, gender and MTX toxicity. However, this needs to be confirmed in further studies. Therapy-related factors that may contribute to heterogeneity in results include differences in dosage and duration of therapy, co-medication and presence of leucovorin rescue. Firstly, in four of the seven studies MTX was given on a weekly basis with doses between 15-30 $\mathrm{mg} / \mathrm{m}^{2}$ for 2-3 years without leucovorin rescue ${ }^{7,9,17,18}$. In the other three studies, including this study 4,16 , MTX was administered at a dose of $4-14 \mathrm{~g} / \mathrm{m}^{2}$. Sub-analysis according to MTX doses did however show absence of effect of the polymorphism in both the low (15-30 $\mathrm{mg} / \mathrm{m}^{2} /$ course) and high dose $\left(4-14 \mathrm{~g} / \mathrm{m}^{2} /\right.$ course) group. Therefore, our hypothesis that the observed difference between ALL and osteosarcoma in the relationship with MTHFR $677 \mathrm{C}>\mathrm{T}$ might be related to the different dosage of MTX, could not be confirmed by the meta-analyses. Secondly, all treatment protocols of patients with ALL consisted of daily oral mercaptopurine (6MP) during maintenance therapy. 6MP influences the pharmacokinetics and pharmacodynamics of MTX; hence, the recorded liver toxicity in ALL patients may be related to but not directly induced by MTX. Unfortunately, there are no reliable measures to determine whether 6MP or MTX is responsible for the development of toxicities. However, a recent study showed that the increased risk of developing toxicity among patients with the 677TT-genotype is rather associated with MTX than 6MP25. Thirdly, leucovorin rescue was applied in our study and that of Patino-Garcia et $\mathrm{al}^{3}$. In the latter study $50 \mathrm{mg} / \mathrm{m}^{2}$ folinic acid was administered every 6 hours up to 12 doses beginning 24 hours post-infusion of MTX. This is substantially higher than the dose used in our study where an initial dose of folinic acid of $15 \mathrm{mg} / \mathrm{m}^{2}$ every 6 hours was followed by leucovorin dose adjustment in accordance with serum MTX-levels at 48-hours post-infusion. This difference in leucovorin rescue may have influenced the effect of a reduced MTHFR activity in patients with 677TT-genotype and might explain the higher hepatotoxicity risk for T-allele carriers in our study and the absence of an increased risk in the study by Patino-Garcia et al ${ }^{3}$.

Based on the analysis of our data on 98 MTX-treated pediatric cancer patients and the meta-analysis we conclude that in patients with cancer the MTHFR 677 T allele has a minor role in the development of MTX-induced hepatotoxicity. These results need to be interpreted cautiously, because they are based on a small number of studies with relatively few incidences of hepatotoxicity, a dichotomized (though uniform) measure of toxicity, and there are indications of heterogeneity between studies, though not statistically significant. However, further study on the effect of leucovorin rescue on MTHFR activity and which patients may benefit from intensive leucovorin rescue is warranted.

\section{References}

Reuben A. Methotrexate controversies. In, Kaplowitz N, DeLeve LD, eds. Drug-induced liver disease. 2nd ed.
New York: Informa Healthcare USA. 2007, pp. 683-705.

Kishi S, Cheng C, French D, et al. Ancestry and pharmacogenetics of antileukemic drug toxicity. Blood.
2007;109:4151-4157.

Trevino LR, Shimasaki N, Yang W, et al. Germline genetic variation in an organic anion transporter polypep-
tide associated with methotrexate pharmacokinetics and clinical effects. I Clin Oncol. 2009; 27:5972-5978.

Patino-Garcia A, Zalacain M, Marrodan $\mathrm{L}$, et al. Methotrexate in pediatric osteosarcoma: response and toxici-
ty in relation to genetic polymorphisms and dihydrofolate reductase and reduced folate carrier 1 expression. Pediatr. 2009;154:688-693.

5 Yang JJ, Cheng $C$, Yang $W_{\text {, et al. Genome-wide interrogation of germline genetic variation associated with }}$ Frost $P$, Blom $H J$, Milos $R$, et al A candidate genetic risk factor for vascular disease: a common mutation in
MTHFR. Nat Genet. 1995;10:111-113.

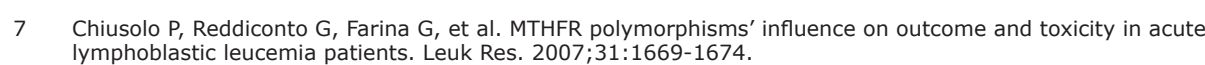

3 Shimasaki N, Mori T, Samejima $\mathrm{H}$, et al. Effects of methylenetetrahydrofolate reductase and reduced folate carrier 1 polymorphisms on high-dose methotrexate-induced toxicities in children with acute lymphoblastic
leukemia or lymphoma. J Pediatr Hematol Oncol. 2006:28:64-68.

Aplenc $R$, Thompson J, Han P, et al. Methylenetetrahydrofolate Reductase polymorphisms and therapy re-
sponse in pediatric acute lymphoblastic leukemia. Cancer Res. 2005:65:2482-2487 10 Seidemann $\mathrm{K}$, Book M, Zimmermann M, et al. MTHFR 677 (C-T) polymorphism is not relevant for prognosis
or therapy-associated toxicity in pediatric NHL: results from 484 patients of multicenter trial NHL-BFM 95 .
Ann Hematol. 2006;85:291-300.

I1 Imanishi $\mathrm{H}$, Okamura $\mathrm{N}$, Yagi $\mathrm{M}$, et al. genetic polymorphisms associated with adverse events and elimina-
tion of methotrexate in childhood acute lymphoblastic leukemia and malignant lymphoma. I Hum Genet. 2007; $52: 166-171$.

12 Whelan J, Seddon B, Perisoglou M. Management of osteosarcoma. Curr Treat Options Oncol. 2006; 7:444-

13 Kamps WA, van der Pal-de Bruin KM, Veerman AJ, et al. Long-term results of Dutch Childhood Oncology
Group studies for children with acute lymphoblastic leukemia from 1984 to 2004. Leukemia. 2010;24:309-
319 . 4 Common toxicity criteria ve

15 De Mattia E, Toffoli G. C677T and A1298C MTHFR polymorphisms, a challenge for antifolate and fluoropyrimDe Mattia E, Toffoli G. C677T and A1298C MTHFR polymorphisms, a chal
dine-based therapy personalization. Eur J Cancer. 2009;45:1333-1351.

16 Gemmati D, Ongaro A, Tognazzo S, et al. Methylenetetrahydrofolate reductase C677T and A1298C gene 2007;92(4):478-485.

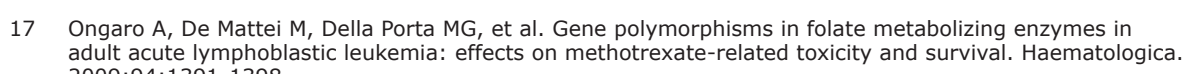
2009;94:1391-1398.

Costea I, Moghrabi A, Laverdier C, et al. Folate cycle gene variants and chemotherapy toxicity in pediatric
patients with acute lymphoblastic leukemia. Haematologica. 2006; $91: 1113-1116$.

Guttormsen AB, Schneede J, Ueland PM, et al. Kinetics of total plasma homocysteine in subjects with hyper-
homocysteinemia due to folate or cobalamin deficiency. Am J Clin Nutr. 1996;63:194-202.

Chiusolo P, Giammarco $S$, Bellesi S, et al. The role of MTHFR and RFC1 polymorphisms on toxicity and outChiusolo P, Giammarco $\mathrm{S}$, Bellesi S, et al. The role of MTs trR and RFC1
come of adult patients with hematological malignancies treated with hi
leucovorin rescue. Cancer Chemother Pharmacol. 2012;69:691-696.

21 Bagley PJ, Selhub J. A common mutation in the methylenetetrahydrofolate reductase gene is associated with
an accumulation of formylated tetrahydrofolates in red blood cells. Proc Natl Acad Sci USA. 1998; $95: 13217-$

22 Brattstrom $L$, Wilcken $D E$, Ohrvik $\mathrm{J}$, et al. Common methylenetetrahydrofolate reductase gene mutation leads to hyperhom
$2520-2526$. 
23 Shi Q, Zhang Z, Li G, et al. Sex differences in risk of lung cancer associated with methylene-tetrahydrofolate reductase polymorphisms. Cancer Epidemiol Biomarkers Prev. 2005;14:1477-1484.

24 Liu A, Menon S, Colson NJ, et al. Analysis of the MTHFR C677T variant with migraine phenotypes. BMC Res Notes. 2010;3:213.

25 Shimasaki N, Mori T, Torii C, et al. Influence of MTHFR and RFC1 polymorphisms on toxicities during maintenance chemotherapy for childhood acute lymphoblastic leukemia or lymphoma. J Pediatr Hematol Oncol. 2008; 30:347-532. 


\section{CHAPTER 6B}

\section{Is there a role for the MTHFR $677 \mathrm{C}>\mathrm{T}$ and the $1298 \mathrm{~A}>\mathrm{C}$ polymorphism in methotrexate induced liver toxicity?}

D. Maroeska W.M. te Loo

Melanie M. Hagleitner

Marieke J.H. Coenen

Pharmacogenomics. 2014;15:1401-1403

Editorial 


\section{Methotrexate treatment}

Methotrexate (MTX) is an antimetabolite used in the treatment of different types of cancer and in a number of autoimmune diseases such as rheumatoid arthritis (RA). Interestingly, the dosage used to treat patients varies substantially between the different types of diseases. Depending on the dosage given several forms of toxicity can be expected e.g. mucositis, bone marrow suppression and liver toxicity. These MTX-induced toxic effects can be reversed by the administration of leucovorin (5-formyltetrahydrofolate). This socalled leucovorin rescue is part of the standard treatment when high doses of MTX are used which is mostly the case in the treatment of cancer and recommended in the treatment of autoimmune diseases where low doses MTX are given. Especially in cancer patients, MTX is given because of its ability to inhibit the folate pathway enzymes including the enzymes dihydrofolatereductase (DHFR) and methylenetetrahydrofolate reductase (MTHFR). The last one, and especially the gene coding for the MTHFR enzyme, has been studied several times regarding the relationship between toxicities caused by MTX and genetic variants present in this gene. It has been suggested that genetic variants of the MTHFR gene are associated with MTX induced liver toxicity, bone marrow suppression and the appearance of mucositis. The two most commonly studied genetic variants are the MTHFR $677 \mathrm{C}>\mathrm{T}$ and 1298 A > C. Both variants are known to result in lower enzyme activity, which is probably the underlying cause of MTX related toxicity.

\section{Clinical relevance of the $677 \mathrm{C}>\mathrm{T}$ and the 1298A >C polymorphism in MTX induced liver toxicity}

Genetic association studies into the role of MTHFR and MTX-induced toxicities are mainly performed in patients with RA and cancer, therefore we will focus on these two diseases. There are several studies describing an association between the $677 \mathrm{C}>\mathrm{T}$ genetic variant of the MTHFR gene and liver toxicity. However, there are also several studies showing no association at all. One of the main problems of these genetic studies are the number of patients included, heterogeneity of the study population, differences in ethnicity and the paucity of prospective studies with adequate toxicity registrations. The last one is in our opinion extremely important to definitely determine if the two most described genetic variants of the MTHFR gene are of clinical importance. Another way to gain insight whether genetic variants in the MTHFR gene are important in the development of toxicities is by combining several small scale studies in a meta-analysis. A PubMed search using the keywords: 'MTHFR', 'toxicity or hepatotoxicity' and 'meta-analysis' revealed six different meta-analysis regarding the role of the $677 \mathrm{C}>\mathrm{T}$ and the $1298 \mathrm{~A}>\mathrm{C}$ variants in the MTHFR gene and MTX induced (liver) toxicity.
Fisher and colleagues performed a meta-analysis in RA patients ${ }^{1}$. They included in total eight different studies in which the $677 \mathrm{C}>\mathrm{T}$ and $1298 \mathrm{~A}>\mathrm{C}$ genetic variants were studied in relationship to MTX induced toxicity. In total 1400 patients were included in the analyses of the $677 \mathrm{C}>\mathrm{T}$ variant and half of them were analyzed regarding the $1298 \mathrm{~A}>\mathrm{C}$ variant Only a significant association could be found between the $677 \mathrm{C}>\mathrm{T}$ variant and overal toxicity. The meta-analysis of Lee and colleagues ${ }^{2}$ included 1514 RA patients to study the relevance of the $677 \mathrm{C}>\mathrm{T}$ and $1298 \mathrm{~A}>\mathrm{C}$ polymorphisms of MTHFR. They did not find any association between the occurrences of MTX induced toxicity and these genetic variants. Unfortunately, although the cohort of patients studied in both meta-analyses was relatively large, there was substantial heterogeneity between the patients cohorts included, which makes it difficult to definitely rule out a role of the MTHFR gene in MTX induced toxicities. Furthermore, in both meta-analyses it is not clear what kind of toxicities were studied as it is mentioned that any toxicity was the end point. In addition, differences in toxicity grading systems between the studies, differences in dosages of MTX used and finally differences in folate rescue scheme make that these meta-analysis cannot be used to answe the question whether these genetic variants of the MTHFR gene are of clinical importance. Therefore meta-analyses performed in more homogenous groups of patients, receiving similar dosages of MTX and using same toxicity grading systems e.g. when MTX induced liver toxicity is being studied should be performed.

A recent meta-analysis study by Owen and colleagues did follow such an approach ${ }^{3}$; they included 309 RA patients with well-defined toxicities due to MTX treatment. No association between the 2 functional MTHFR genetic variants and the occurrence of liver toxicity or other toxicities were found. Although this cohort was smaller than the previously mentioned studies, the cohort was homogenous, well defined regarding toxicity and similar ethnicity and is therefore very useful for a genetic association study. The clinical relevance of these data within the field of RA treatment is however debatable. In patients with RA dosages of MTX are much lower than those for cancer treatment. It is generally thought that especially these higher dosages of MTX are the trigger for MTX induced liver toxicity. For this reason, it is of utmost interest whether there are meta-analyses performed in cancer patients receiving these high dosages of MTX. Indeed, there are few genetic association studies investigating cancer patients for a relation between the MTHFR gene and MTX induced toxicities. First of all, the study of Yang and colleagues ${ }^{4}$ including a cohort of patients with acute lymphoblastic leukemia, both adults and children, with different ethnicities and found a significant association between the $677 \mathrm{C}>\mathrm{T}$ genetic variant and the occurrence of liver toxicity. In their statistical analyses they stratified patients regarding their ethnicity demonstrating that especially in Africans and Caucasians the risk of liver toxicity was high whereas Asians were not at risk. Interestingly, they also performed a stratification regarding age and found that the occurrence of liver toxicity in association with the $677 \mathrm{C}>\mathrm{T}$ variant was predominantly found in adults and not in children. This is very interesting as in the treatment of childhood acute lymphoblastic leukemia (ALL) high dosages 
(3-5 $\left.\mathrm{g} / \mathrm{m}^{2}\right)$ of MTX are used and in adults not $\left(15-30 \mathrm{mg} / \mathrm{m}^{2}\right)$. However, the number of patients studied was too small to make any definite conclusions. In a recent meta-analysis study performed by Lopez-Lopez and colleagues ${ }^{5}$, children with ALL were investigated regarding the presence of the $677 \mathrm{C}>\mathrm{T}$ and $1298 \mathrm{~A}>\mathrm{C}$ genetic variant of the MTHFR gene and the occurrence of MTX induced toxicity. They showed, as was suggested by the study of Yang and colleagues, that in this pediatric population, there is a lack of association between these genetic variants in children and MTX induced toxicity. Finally, a meta-analysis performed by our own group included more than 1000 patients with osteosarcoma and $\mathrm{ALL}^{6}$. They all received high dosages of MTX. Primary goal of this study was to answer the question whether or whether not the $677 \mathrm{C}>\mathrm{T}$ variant is associated with the risk for liver toxicity due to MTX treatment. In all patients included, hepatotoxicity was evaluated retrospectively and uniformly classified according the National Cancer Institute criteria for adverse events. No association was found between the presence of the genetic variant in the MTHFR gene and the development of liver toxicity. Although the cohort was large enough, all patients received high dose MTX and toxicities were reclassified using the same criteria for adverse events, this study has its limitations by its retrospective character, including patients treated in different countries and using different regimens of supportive care such as leucovorin rescue. Notably, in all meta-analysis studies discussed in this editorial, several single cohort studies were repeatedly included in the different meta-analyses. Therefore, to finally proof the association between genetic variants in MTHFR and MTX induced toxicity, a large prospective study, including a homogenous group of patients, treated according the same protocols and prospectively classified according the same toxicity criteria is necessary.

\section{Conclusions and further recommendations}

Based on all studies performed so far, including the meta-analyses present in literature, one must conclude that it is still unclear whether the two most studied genetic variants (677C $>$ T and $1298 \mathrm{C}>A$ ) of the MTHFR gene, play a clinical significant role in the development of MTX induced liver toxicity. Most meta-analysis performed showed no association between variants in MTHFR and MTX induced toxicities, suggesting that if there is a role of the MTHFR variants this will be very limited with questionable clinical relevance. Most probably MTX related toxicities have a multifactorial background meaning that they are induced by a combination of genetic and environmental factors. The pharmacological metabolism of MTX involves many transporters and enzymes that can influence MTX efficacy and toxicity. Future studies should focus more on these genes. Ultimately, if large enough patient cohorts are collected a hypothesis driven genetic approach would be the method of choice (e.g. genome-wide association study or next generation sequencing). Such study will allow the identification of genes associated with MTX related toxicities beyond the obvious candidate genes. In addition, it should be realized that the clinical relevance of pharmacogenetic studies can only be determined in large enough patient cohorts, followed by proper replication of identified associations and preferably by performing a prospective study.

\section{Summary}

Although many studies have been performed regarding the role of the $677 \mathrm{C}>\mathrm{T}$ and the 1298C > A genetic variant versus MTX induced liver toxicity, no clear conclusion can be made yet. Most meta-analyses performed demonstrate that there is no clear relationship between these genetic variants of the MTHFR gene and liver toxicity although there are meta-analyses suggesting there might be a minor role. It is clear that before pharmacogenetic results can be used for clinical practice, studies are being performed in large homogenous patient cohorts followed by replication of the results to identify the true value of the association found. 


\section{References}

1 Fisher MC, Cronstein BN. Metaanalysis of methylenetetrahydrofolate reductase (MTHFR) polymorphisms affecting methotrexate toxicity. J Rheumatol. 2009;36;539-545.

2 Lee YH, Song GG. Associations between the C677T and A1298C polymorphisms of MTHFR and the efficacy and toxicity of methotrexate in rheumatoid arthritis: a meta-analysis. Clin Drug Investig. 2010;30:101-108.

3 Owen SA, Lunt M, Bowes J, et al. MTHFR gene polymorphisms and outcome of methotrexate treatment in patients with rheumatoid arthritis: analysis of key polymorphisms and meta-analysis of C677T and A1298C polymorphisms. Pharmacogenomics J. 2013;13:137-147.

4 Yang L, Hu X, Xu L. Impact of methylenetetrahydrofolate reductase (MTHFR) polymorphisms on methotrexate-induced toxicities in acute lymphoblastic leukemia: a meta-analysis. Tumor Biol. 2012;33:1445-1454.

5 Lopez-Lopez E, Martin-Guerrero I, Ballesteros J, et al. A systematic review and meta-analysis of MTHFR polymorphisms in methotrexate toxicity prediction in pediatric acute lymphoblastic leukemia. Pharmacogenomics J. 2013;13:498-506.

6 Hagleitner MM, Coenen MJ, Aplenc R, et al. The role of the MTHFR 677C >T polymorphism in methotrexate-induced liver toxicity: a meta-analysis in patients with cancer. Pharmacogenomics J. 2014;14:115-119. 


\section{CHAPTER 7}

\section{Influence of genetic variants in TPMT and COMT associated with cisplatin induced hearing loss in patients with cancer: two new cohorts and a meta-analysis reveal significant heterogeneity between cohorts}

Melanie M. Hagleitner

Marieke J.H. Coenen

Ana Patino-Garcia

Eveline S.J.M. de Bont

Anna Gonzalez-Neira

Hanneke I. Vos
Frank N. van Leeuwen

Hans Gelderblom

Peter M. Hoogerbrugge

Henk-Jan Guchelaar

D. Maroeska W.M. te Loo 


\section{Abstract}

Treatment with cisplatin-containing chemotherapy regimens causes hearing loss in 40$60 \%$ of cancer patients. It has been suggested that genetic variants in the genes encoding thiopurine S-methyltransferase (TPMT) and catechol O-methyltransferase (COMT) can predict the development of cisplatin-induced ototoxicity and may explain interindividual variability in sensitivity to cisplatin-induced hearing loss. Two recently published studies however, sought to validate these findings and showed inconsistent results. The aim of this study was to evaluate the role of polymorphisms in the TPMT and COMT genes in cisplatin-induced ototoxicity. Therefore we investigated two independent cohorts of 110 Dutch and 38 Spanish patients with osteosarcoma and performed a meta-analysis including all previously published studies resulting in a total population of 664 patients with cancer With this largest meta-analysis performed to date, we show that the influence of TPMT and COMT on the development of cisplatin-induced hearing loss may be less important than previously suggested.

\section{Introduction}

Cisplatin is an effective chemotherapeutic drug for several types of cancer such as ovarian cancer, lung cancer, osteosarcoma and neuroblastoma. Ototoxicity, characterized as a permanent, bilateral sensorineural hearing loss, is one of the most common side effects of cisplatin and occurs in $40-60 \%$ of patients ${ }^{1}$. Ototoxicity is one of the main reasons for dose reduction or termination of treatment with cisplatin treatment. Clinical risk factors for the development of cisplatin-induced ototoxicity have been described such as age younger than 5 years at diagnosis ${ }^{2,3}$, concurrent medication like carboplatin ${ }^{4}$ as well as higher cumulative doses ${ }^{2,4}$, cranial radiation ${ }^{5}$ and pre-existing renal dysfunction. Nevertheless, these clinical factors are not sufficient to reliably predict ototoxicity before the start of treatment. A candidate gene study in 166 pediatric patients suggested that genetic variants in the genes encoding thiopurine S-methyltransferase (TPMT) and catechol O-methyltransferase (COMT) can predict the development of cisplatin-induced ototoxicity and may explain the interindividual variability ${ }^{6}$. Two recently published studies, developed to validate these findings, showed inconsistent results. Pussegoda and colleagues confirmed the findings of Ross et al. for variants in the TPMT gene in a cohort of 155 patients however, with smaller effect sizes compared to the original findings ${ }^{7}$. In contrast, no association of TPMT or COMT and ototoxicity was found in a cohort of 213 patients with medulloblastoma ${ }^{8}$. Based on these three studies no recommendation for clinical implementation regarding genetic variants in TPMT and COMT and ototoxicity can be made. The aim of the current study is to provide a clearer picture of the genetic impact of TPMT and COMT variants on developing cisplatin-induced ototoxicity. Therefore we investigated two independent cohorts of 110 Dutch and 38 Spanish patients with osteosarcoma and performed a meta-analysis including previously published studies ${ }^{6-8}$ resulting in a total population of 664 patients with cancer. 


\section{Materials and methods}

\section{Dutch cohort}

A cohort of 110 Dutch patients with high-grade osteosarcoma treated with cisplatin was recruited from the Radboud University Medical Center, the University Medical Center of Groningen and Leiden University Medical Center. All patients were treated with cisplatin with a median cumulative dose of $500 \mathrm{mg} / \mathrm{m}^{2}$ (range: 100 to $600 \mathrm{mg} / \mathrm{m}^{2}$ ). Of patients included audiometric analysis were available at diagnose, during therapy and after completion of therapy. Data concerning administration of other potentially ototoxic medications such as furosemide, vancomycin, gentamicin, tobramycin, amphotericin B, carboplatin and vincristine, were recorded. In patients alive, DNA was extracted from blood using the QIAamp DNA Blood Midi kit (Qiagen Venlo, The Netherlands) or saliva ( $2 \mathrm{ml}$ ) using the Oragene DNA purification protocol (DNA Genotek, Kanata, Ontario, Canada). Of patients that passed away, $(\mathrm{N}=39,26.5 \%$, median follow up time 2.3 years with range 1.1-5.5 years), DNA of paraffin-embedded samples was extracted as recently described ${ }^{9}$. The study was approved by the ethics committee from the Radboud University Medical Center as the central committee for this study for the Netherlands and written informed consent was obtained from parents and/or patients

\section{Spanish cohort}

To enlarge sample size for meta-analyses, we additionally included an independent Spanish cohort of osteosarcoma patients who were treated with cisplatin-containing regimens To prevent population bias, this cohort was analyzed separately. In total, 38 patients with osteosarcoma were included on the basis of availability of germline DNA and audiologic assessment at least one month after initial treatment. Data regarding ototoxic medication were available. The study was approved by the local ethics committee from the University of Navarra and University Clinic in Pamplona. Written informed consent was obtained from parents and/or patients.

\section{Ototoxicity}

In both cohorts audiologic evaluations were prospectively performed at diagnosis, during therapy and after completion of therapy. First audiological follow-up was performed 1-3 months after completion of therapy and thereafter annually. All audiometric assessments were age appropriate performed by conventional or play audiometry under standardized conditions as part of routine clinical monitoring for cisplatin-related hearing loss. Hearing loss was retrospectively classified according to two grading systems: the National Cancer Institute CTCAE version 3.0 (http://ctep.cancer.gov/forms/CTCAEv3.pdf) and the new SIOP Boston ototoxicity scale which classifies hearing loss on the basis of absolute hearing levels in 4 grades ${ }^{10}$. The most recent audiologic assessment during follow-up period after the last cisplatin course was used for analysis.

\section{Genotyping}

Previously associated variants in TPMT (rs12201199: C_31923406_10, rs1800460: C_30634116_20, rs1142345: C__19567_20) and COMT (rs4646316: C_29193982_10, rs9332377: C__29614343_10) were genotyped using Taqman allelic discrimination assays according to the protocol of the manufacturer (Invitrogen, Bleiswijk The Netherlands). After amplification, the fluorescent signal for allelic discrimination was determined using the 7500 Fast Real-time System (Invitrogen). Automated allele calling was performed by allelic discrimination plots using SDS 1.4 software (Invitrogen).

\section{SNP analysis}

In both cohorts, statistical differences regarding demographic data between patients who developed hearing loss (cases; $>20 \mathrm{~dB}$ hearing loss above $4 \mathrm{kHz}$ ) and patients without hearing loss (controls; $\leq 20 \mathrm{~dB}$ hearing loss at all frequencies) were assessed by the Fischer exact test. Reported $\mathrm{p}$-values are two-sided and are considered statistically significant if $<0.05$. Associations between ototoxicity and potential confounders, such as age, gender, cumulative dosage of cisplatin and concomitant drugs were evaluated using a $2 \times 2$ table (in case of dichotomized variables) or linear regression (in case of linear variables) in SPSS version 20 (SPSS Inc., Chicago, Il). To assess the effect of the genetic variants on ototoxicity data were dichotomized in grade 0 vs. grade 2-4. Multivariate logistic regression analyses included vincristine exposure as confounder in PLINK ${ }^{11}$ using the command-logistic. 


\section{Meta-analyses}

We searched PubMed for papers using the keywords: 'ototoxicity', 'TPMT' and 'COMT'. Since the publication of Ross and colleagues ${ }^{6}$ in 2009 only 2 other studies ${ }^{7,8}$ evaluated the association of the COMT and TPMT variants in cancer patients. Meta-analysis was performed on these 3 previously published studies and the 2 cohorts presented in this study were included. Of each of the 5 studies the CTCAE hearing loss criteria were used, excluding patients with grade 1 ototoxicity to better differentiate between cisplatin-induced ototoxicity and normal hearing. The studies were weighed using the inverse variance method, where larger studies with smaller standard errors have more weight than smaller studies with larger errors (Review manager 5.0, The Cochrane Collaboration, Oxford, UK). Heterogeneity among studies was examined with $\mathrm{I}^{2}$ statistics that can be interpreted as the proportion of total variation contributed by between-study variation. Allelic odds ratio (OR) were recalculated dependent on the number of cases and controls in each cohort and $95 \%$ confidence interval (CI) were estimated using a fixed effects model or random effects models in case large heterogeneity $\mathrm{I}^{2}>50 \%$ was observed.

\section{Results}

\section{Dutch cohort}

A total of 110 patients with Dutch ancestry and newly diagnosed osteosarcoma were included of whom 42 (38.2\%) developed $>20 \mathrm{~dB}$ hearing loss above $4 \mathrm{kHz}$. Characteristics of patients divided in patients with hearing loss (cases; $>20 \mathrm{~dB}$ hearing loss above $4 \mathrm{kHz}$ ) and patients without hearing loss (controls; $\leq 20 \mathrm{~dB}$ hearing loss at all frequencies) are depicted in Table I.

Baseline characteristics did not show statistically significant differences between the 2 groups. None of the patients included was treated with cranial irradiation or with concomitant ototoxic antibiotics. Only 5 patients were additionally treated with vincristine. Othe ototoxic chemotherapeutics were not administered. In 23 (20.9\%) of all patients cumulative dose of cisplatin was reduced due to ototoxicity $(\mathrm{N}=9,>20 \mathrm{~dB}$ hearing loss above 4 $\mathrm{kHz})$ or other cisplatin-induced toxicity $(\mathrm{N}=14)$. Baseline audiograms were available in 87 patients $(79 \%)$ showing normal baseline hearing thresholds ( $\leq 20 \mathrm{~dB}$ ) in all patients. Median follow up period was 5.2 years (range 23 to 7763 days). Different ototoxicity grading systems were used to exclude biased dichotomisation. For the CTCAE criteria, 80 patients with baseline audiogram were included. Patients with grade 1 ototoxicity $(N=7)$ were excluded in order to use same inclusion/exclusion criteria as previous studies in literature ${ }^{6-8}$. Classification according to the CTCAE criteria showed in all but 7 patients' identical toxicity grades when compared to the SIOP grading system. Four patients with grade 1 and 3 patients with grade 2 ototoxicity according to the SIOP Boston ototoxicity scale were upgraded to grade 2 and 3 according the CTCAE criteria. Hearing loss grade 2-4 was seen in 23 patients graded according the CTCAE criteria ( 0 vs. $>25 \mathrm{~dB}$ at $4-8 \mathrm{kHz}$ ) and 22 according to the SIOP criteria ( 0 vs. $>20 \mathrm{~dB}$ at $4-8 \mathrm{kHz}$ ). Univariate analysis showed no association with non-genetic factors previously reported to be associated with ototoxicity: age $(p=0.73)$ and cumulative dose of cisplatin $(p=0.99)$.

\section{Spanish cohort}

To enlarge sample size for the meta-analyses we included another cohor of 38 Spanish patients of European ancestry with osteosarcoma (Table I). No statistically significant differences concerning age, gender or cumulative dose of cisplatin was seen between patients with hearing loss (cases; $>20 \mathrm{~dB}$ hearing loss above $4 \mathrm{kHz}$ ) and patients with normal hearing (controls; $\leq 20 \mathrm{~dB}$ hearing loss at all frequencies). None of the included patients was additionally treated with cranial irradiation. Regarding concomitant medication, all but 6 patients were additionally treated with vincristine and 23 patients received ototoxic antibiotics, such as vancomycin, gentamicin or tobramycin. In univariate analysis the use of these antibiotics was not a confounding factor to develop ototoxicity in this cohort $(p=0.74)$. As to the small sample size and to prevent population bias due to ethnicity, genotyping results of this cohort were analyzed as an independent cohort in the meta-analyses. 


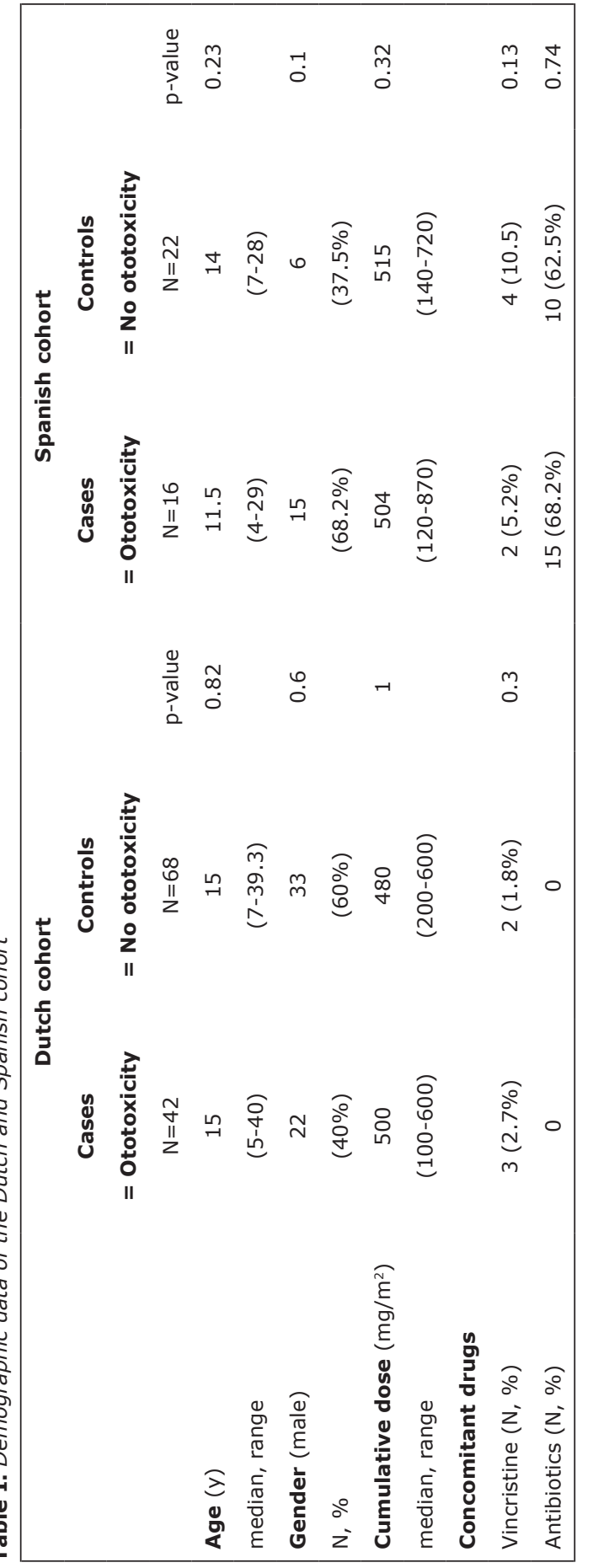

\section{Genotyping}

We genotyped three variants in the TPMT gene (rs12201199, rs1800460, and rs1142345) and two in the COMT gene (rs4646316, rs9332377). No deviation from Hardy-Weinberg equilibrium was observed. Irrespective of the grading system used (CTCAE or SIOP), association analysis using an additive genetic model correcting for usage of vincristine, showed no association between genetic variants in TPMT or COMT with an increased risk of ototoxicity in the Dutch cohort (Table II and Table III).

Table II. Association analyses of the Dutch cohort - SIOP vs. CTCAE criteria

\begin{tabular}{|llcccccc|}
\hline & & \multicolumn{3}{c}{ SIOP Boston criteria } & \multicolumn{3}{c|}{ CTCAE criteria } \\
Gene & SNP & OR & $\mathbf{9 5 \%}$ CI & p-value & OR & $\mathbf{9 5 \%} \mathbf{~ C I}$ & p-value \\
TPMT & rs1142345 & 0.96 & $0.30-3.08$ & 0.95 & 0.71 & $0.15-3.26$ & 0.66 \\
& rs1800460 & 0.49 & $0.12-1.97$ & 0.31 & 0.32 & $0.04-2.58$ & 0.28 \\
& rs12201199 & 0.65 & $0.22-1.91$ & 0.44 & 0.74 & $0.20-2.76$ & 0.65 \\
COMT & rs4646316 & 0.49 & $0.22-1.14$ & 0.1 & 0.67 & $1.81-0.24$ & 0.42 \\
& rs9332377 & 0.8 & $0.41-1.55$ & 0.51 & 0.91 & $0.41-2.04$ & 0.83 \\
\hline
\end{tabular}

Table III. Call rates of the Dutch and Spanish cohort for each variant according to the CTCAE

\begin{tabular}{|llcc|}
\hline ototoxicity & criteria \\
\hline Gene & SNP & Dutch Cohort, $\mathbf{N = 8 0}$ & Spanish Cohort, $\mathbf{N = 3 4}$ \\
TPMT & rs1142345 & 69 & 32 \\
& rs1800460 & 70 & 33 \\
& rs12201199 & 72 & 34 \\
COMT & rs4646316 & 71 & 32 \\
& rs9332377 & 80 & 33 \\
\hline
\end{tabular}

\section{Meta-analyses}

So far, 3 studies with 4 independent cohorts studying the influence of TPMT and COMT on the development of ototoxicity have been published ${ }^{6-8}$. With this study we add two additional independent cohorts. In all studies the CTCAE criteria were reported. To better differentiate between cases and controls all studies excluded patients with grade 1 ototoxicity. In total, 664 patients were included in the meta-analyses. Table IV shows the demographic data of the initial studies, as well as the number of patients per study that were included in the meta-analysis. In Figures I and II OR for each individual study are presented. The genetic variant rs 4646316 in the COMT gene was the only significant association with ototoxicity (OR A vs. T allele=1.52, 95\% CI: $1.16-1.99, \mathrm{P}=0.003$; Figure II). The heteroge- 
neity test showed a non-statistically significant heterogeneity with an $\mathrm{I}^{2}$ of $31 \%$. Notably, there are substantial differences in the range of the 'total events' captured between the of Pussegoda and colleagues.

Table IV. Demographic data of patients with ototoxicity included for meta-analyses

\begin{tabular}{|lccccc|}
\hline & $\begin{array}{c}\text { Cases/Total } \\
\text { cohort }\end{array}$ & Age (y) & Gender & $\begin{array}{c}\text { Cumulative } \\
\text { dose }\left(\mathbf{m g} / \mathbf{m}^{2}\right)\end{array}$ & $\begin{array}{c}\text { Cranial } \\
\text { irrdiation }\end{array}$ \\
Ross et al. & $\mathrm{N}$ & Median & $\%$ & Median & $\%$ \\
Pussegoda et al. & $106 / 162$ & 6 & $67 \%$ & $400 \mathrm{mg} / \mathrm{m}^{2}$ & $18.5 \%$ \\
Yang et al., radiation cohort & $87 / 155$ & 6 & $49.4 \%$ & $400 \mathrm{mg} / \mathrm{m}^{2}$ & $18 \%$ \\
Yang et al., no radiation & $131 / 195$ & 7 & $68.8 \% *$ & $300 \mathrm{mg} / \mathrm{m}^{2 *}$ & $100 \% *$ \\
Hagleitner, this study & $35 / 38$ & 8 & $65 \%$ & $500 \mathrm{mg} / \mathrm{m}^{2}$ & $0 \%$ \\
Patino-Garcia, this study & $22 / 80$ & 15 & $40 \%$ & $500 \mathrm{mg} / \mathrm{m}^{2}$ & $0 \%$ \\
\hline
\end{tabular}

*data of total group as published by Yang et al.
Figure I. Forest plots of genetic variants in TPMT gene

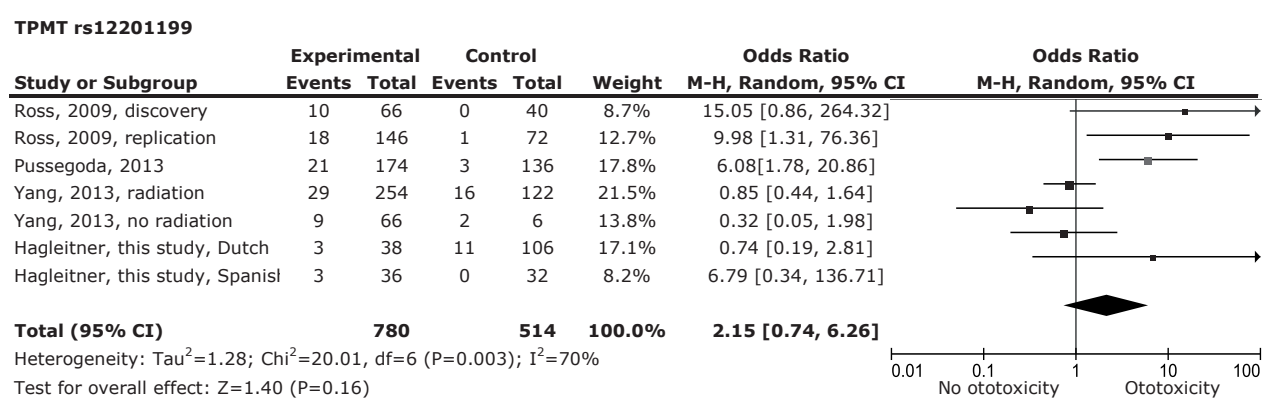

Test for overall effect: $Z=1.40(P=0.16)$

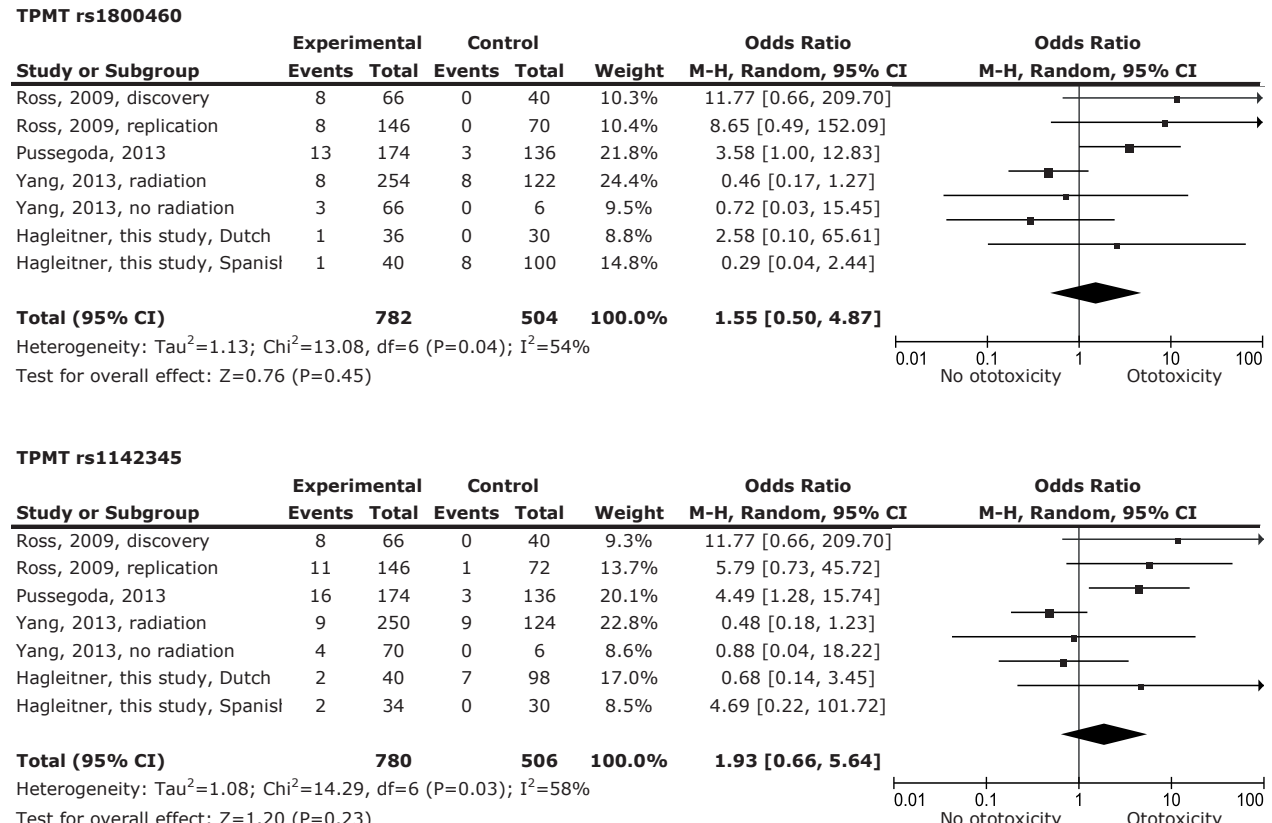

Test for overall effect: $\mathrm{Z}=1.20(\mathrm{P}=0.23)$

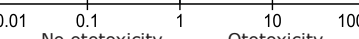


Figure II. Forest plots of genetic variants in COMT gene

СоMT rs4646316

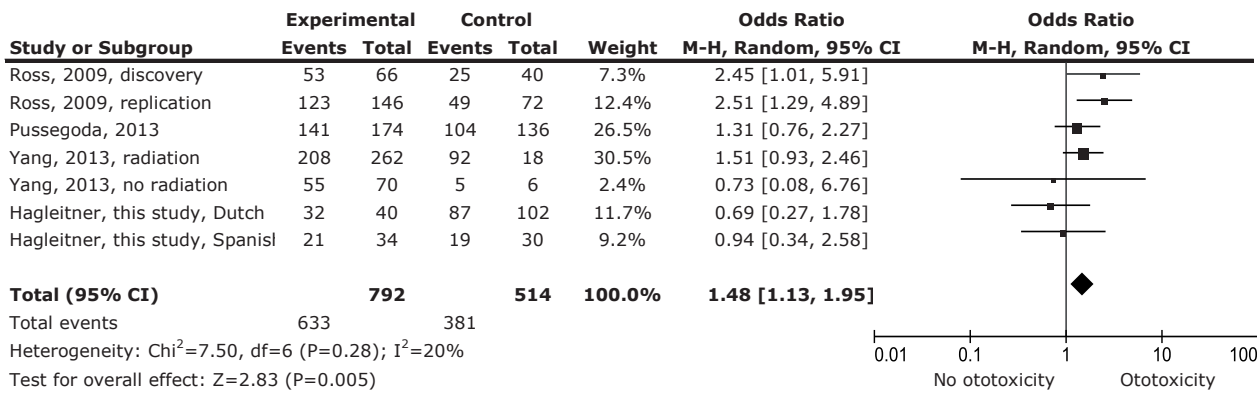

Heterogeneity: Chi $=7.50$, df $=6(\mathrm{P}=0.28) ; \mathrm{I}^{2}=20 \%$

сомт rs9332377

\begin{tabular}{|c|c|c|c|c|c|c|c|}
\hline \multirow{2}{*}{$\begin{array}{l}\text { Study or Subgroup } \\
\text { Ross, 2009, discovery }\end{array}$} & \multicolumn{2}{|c|}{$\begin{array}{l}\text { Experimental } \\
\text { Events Total }\end{array}$} & \multicolumn{2}{|c|}{$\begin{array}{c}\text { Control } \\
\text { Events Total }\end{array}$} & \multirow{2}{*}{$\frac{\text { Weight }}{10.4 \%}$} & \multirow{3}{*}{$\begin{array}{c}\text { Odds Ratio } \\
\text { M-H, Random, } 95 \% \mathrm{CI}\end{array}$} & \multirow{2}{*}{$\begin{array}{c}\text { Odds Ratio } \\
\text { M-H, Random, } 95 \% \text { CI }\end{array}$} \\
\hline & 14 & 66 & 2 & 40 & & & \\
\hline Ross, 2009, replication & 22 & & 2 & 72 & & & \\
\hline Pussegoda, 2013 & 38 & 174 & 23 & 136 & $20.6 \%$ & $1.37[0.77,2.44]$ & \\
\hline Yang, 2013, radiation & 48 & 260 & 21 & 128 & $20.7 \%$ & $1.15[0.66,2.03]$ & \\
\hline Yang, 2013 , no radiation & 9 & 70 & 2 & 6 & & $0.30[0.05,1.85]$ & \\
\hline Hagleitner, this study, Dutch & 10 & 44 & 28 & 116 & $17.6 \%$ & $0.92[0.41,2.11]$ & \\
\hline Hagleitner, this study, Spanis & 3 & 36 & 10 & 30 & $11.5 \%$ & $0.18[0.04,0.74]$ & \\
\hline Total (95\% CI) & & 796 & & 528 & $100.0 \%$ & $1.16[0.61,2.23]$ & \\
\hline $\begin{array}{l}\text { Total events } \\
\text { Heterogeneity: Taü2}=0 .\end{array}$ & 144 & & & & & & \\
\hline
\end{tabular}

\section{Discussion}

Although, the results of Ross and colleagues investigating the influence of variants in TPMT and COMT were very promising, the outcome of more recent studies argues against the premise that these variants might help to identify patients at risk for ototoxicity. In the present study, therefore, we aimed to gain a clearer picture on the role of these variants by adding additional data on the subjects, and by performing a meta-analysis including two new cohorts.

Our Dutch dataset of 110 uniformly treated patients with osteosarcoma, is a homogenous group without potentially confounding factors such as cranial radiation and concomitant medication. For classification of cisplatin-induced hearing loss, we preferred to use the SIOP Boston scale as it combines the two main types of measuring ototoxicity, namely changes of hearing from baseline as well as absolute hearing levels ${ }^{10}$. As previously published studies used the CTCAE criteria for the classification of ototoxicity, we reclassified our data according to the CTCAE criteria, and excluded grade 1 ototoxicity to better distinguish between cases and controls. In the Dutch cohort, and also the other newly analyzed Spanish cohort, we did not observe any statistically significant association between the genetic variants in TPMT, COMT and ototoxicity. We did observe differences between the two cohorts such as lower ototoxicity rate in the Dutch cohort which might be explained by the inclusion of adults until the age of 40 years. It is known that young children are more susceptible to ototoxicity from cisplatin ${ }^{3}$. Furthermore, the Dutch cohort included patients who have already died. It might be that controls that died have become cases if they have survived longer. However, the median follow up time of these patients who have died was 2.3 years with a range between 1.1 and 5.5 years. Studies included in this meta-analysis showed universally that the majority of ototoxicity occurred between 0.5 and 6 months from start of cisplatin chemotherapy ${ }^{6-8}$.

After meta-analyses of the genetic variants in TPMT and COMT including in total $664 \mathrm{pa}$ tients only rs 4646316 variant in the COMT gene remained statistically significant. However, the effect was much smaller than reported in previously published studies. In the initia discovery cohort of Ross and colleagues the OR of this variant to develop ototoxicity was 2.5 (95\% CI: 1.5-4.3) ${ }^{6}$. Whereas the combined effect of all studies included in the meta-analysis showed an OR of 1.52 (95\% CI: 1.16-1.99). Three of the cohorts (excluding the initial study by Ross et al.) showed the same direction of effect for rs4646316, however, this was not statistically significant. The COMT gene encodes for the COMT enzyme which is involved in the inactivation of catecholamine neurotransmitters. Although, it has been shown that it is also highly expressed in sensory hair cells of the inner ear, its role regarding auditory function remains unclear ${ }^{12}$. In addition, the influence of the variant rs4646361 on the COMT enzyme has not been studied yet.

Generally, the association between TPMT variants and cisplatin ototoxicity was unexpect$\mathrm{d}^{9}$, as it was not previously linked to cisplatin metabolism. Recent published data support the hypothesis that TPMT might have an influence on cisplatin metabolism by increasing 
levels of S-adenosylmethionine (SAM) $)^{13}$. Furthermore, a recent study demonstrated that functional TPMT variants were associated with progression-free survival in cisplatin-based chemotherapy outcomes in ovarian cancer ${ }^{14}$. However, in vivo studies in mice with different TPMT genotypes showed no differences in hearing damage between TPMT wild-type and knockout mice ${ }^{8}$.

With the largest meta-analysis performed to date we show that the influence of TPMT and COMT on the development of cisplatin-induced hearing loss may be less pronounced than previously suggested. This lack of association observed might be attributable to heterogeneity between the different studies as shown in Table II. First, different patient populations with different ethnicities were used in the studies which could account for the differences in the genetic associations in the meta-analysis. Second, in the study cohorts with clear positive association, a heterogeneous group of patients with respectively 9 and 12 different types of cancers was included ${ }^{6,7}$. As a consequence, patients were treated with differen treatment regimens sometimes including cranial irradiation and different co-medication. In these studies an analysis for specific patient subgroups was not performed probably due to the limited number of patients included for each subgroup. Also the cohorts with clear negative association included patients with different cancer types, one study included patients with osteosarcoma (this study) as in the study of Yang et al. patients with medulloblastoma have been included which necessitates highly different treatment protocols regarding cumulative dose of cisplatin, cranial radiation and concomitant medication. Third, the different types of cancer were not equally distributed among patients with and without ototoxicity, for example in the cohort of solid tumors investigated by Yang and colleagues 35 of the 38 patients $^{8}$ were reported to have hearing loss which may lead to selection bias. Fourth, the median cumulative doses of cisplatin ranged from 300 to $500 \mathrm{mg} / \mathrm{m}^{2}$ in the different studies. However, neither in the study cohort of medulloblastomas with a relatively low cumulative dose of cisplatin ${ }^{8}$ nor in osteosarcoma patients treated with higher doses of cisplatin (this study), an association was found. In addition, young age has recently been pointed out as an important factor to develop cisplatin-induced hearing loss $^{3}$, which may be the reason for the lower incidence of ototoxicity in the osteosarcoma cohort as the median age at diagnosis is older compared to the other cohorts. At last, the use of otoprotective agents, such as amifostine, may be a potentially confounding factor. A Cochrane study on this topic was due to methodological limitations of individual studies unable to show evidence of effect to use amifostine as an otoprotective intervention ${ }^{15}$. However, there was also no evidence that it had no effect as otoprotectant. In the study of Yang et al the use of amifostine was linked to less ototoxicity.

It is clear that all studies included in this meta-analysis have confounding variables which could potentially influence the development of ototoxicity. This is important given the inverse method of weighing studies which has been used in this meta-analysis. The studies of Pussegoda et al and Yang et al were consistently attributed the highest weighting Pussegoda et al included a diverse population of different cancers with different treatment protocols, the youngest population in this meta-analysis with different follow-up time between cases ( 5 years follow-up) and controls ( 2 years follow-up). The radiation cohort of Yang et al patients received highly ototoxic cranial irradiation, but $90 \%$ also received an otoprotectant, and overall these patients received a significantly lower cumulative dose of cisplatin. Thus, the weight attributed to a study may affect the results of the meta-analysis, however this is something that cannot be circumvented in this type of analysis.

Nevertheless, younger age and higher cumulative doses of cisplatin appear to be the most consistent risk factors for ototoxicity. However, an upfront risk stratification based on these two factors is unreliable as these factors seem to be protocol specific and dose adjustments may jeopardize treatment efficacy of cisplatin. The addition of otoprotective agents in combination with cisplatin might be an alternative treatment strategy to prevent hearing loss. Although, the mechanism of cisplatin-induced hearing loss is not fully understood, severa preclinical studies have shown that in the cochlear cells cisplatin induces the production of toxic levels of reactive oxygen species (ROS) which can initiate cochlear cell death leading to hearing loss ${ }^{16,17}$. Numerous studies have investigated a variety of antioxidant agents to protect cochlea cells from cisplatin damage. Among them, sodium thiosulfate, D- or L-methionine, glutathione ester and amifostine were successfully tested in animal models ${ }^{18,19}$ The efficacy of otoprotective agents in patients is however disappointing. For instance, sodium thiosulfate showed an unwanted compromised antitumor effect of cisplatin ${ }^{20}$ and no otoprotection was observed in children with germ cell tumors treated with amifostine in combination with cisplatin ${ }^{21}$.

It remains challenging to find the right balance to maintain antitumor effect of cisplatin and on the other hand to prevent unwanted adverse effects. Innovative studies translating basic science into clinical practice are needed to unravel both, the mechanism of cisplatin-induced hearing loss and identification of patients at risk to develop ototoxicity. Pharmacogenetics may help to find new target genes, unfortunately it seems that the genes TPMT and COMT play only a minor role in cisplatininduced ototoxicity and should therefore not guide clinical decision making for cisplatin dosing. 


\section{References}

1 Knight KR, Kraemer DF, Neuwelt EA: Ototoxicity in children receiving platinum chemotherapy: underestimating a commonly occurring toxicity that may influence academic and social development. J Clin Oncol 2005; 23:8588-8596.

2 Li Y, Womer RB, Silber JH: Predicting cisplaitn ototoxicity in children: the influence of age and the cumulative dose. Eur J Cancer 2004;40:2445-2451.

3 Yancey A, Harris MS, Egbelakin A, et al: Risk Factors for Cisplatin-Associated Ototoxicity in Pediatric Oncology Patients. Pediatr Blood Cancer 2012;59:144-148.

4 Blakley BW, Gupta AK, Myers SF, et al: Risk factors for ototoxicity due to cisplatin. Arch Otolaryngol Head Neck surg 1994;120:541-546.

5 Pan CC, Eisbruch A, Lee JS, et al: Prospective study of inner ear radiation dose and hearing loss in head-andneck cancer patients. Int J Radiat Oncol Biol Phys 2005;61:1393-1402.

6 Ross $\mathrm{CJ}$, Katzov-Eckert $\mathrm{H}$, Dubé MP, et al: Genetic variants in TPMT and COMT are associated with hearing loss in children receiving cisplatin chemotherapy. Nat Genet 2009;41:1345-1349.

7 Pussegoda $\mathrm{K}$, Ross $\mathrm{CJ}$, Visscher $\mathrm{H}$, et al: Replication of TPMT and ABCC3 genetic variants highly associated with cisplatin-induced hearing loss in children. Clin Pharmacol Ther 2013;94:243-251.

8 Yang JJ, Lim JY, Huang J, et al: The role of inherited TPMT and COMT genetic variation in cisplatin-induced ototoxicity in children with cancer. Clin Pharmacol Ther 2013;94:252-259.

9 Hagleitner MM, Coenen MJ, Jeuken JW, et al:Taqman genotyping assays can be used on decalcified and paraffin-embedded tissue from patients with osteosarcoma. Pediatr Blood Cancer 2011;56:35-38

10 Brock PR, Knight KR, Freyer DR, et al: Platinum-induced ototoxicity in children: a consensus review on mechanisms, predisposition, and protection, including a new International Society of Pediatric Oncology Boston ototoxicity scale. J Clin Oncol 2012;30:2408-2417.

11 Purcell S, Neale B, Todd-Brown K, et al: PLINK: a tool set for whole-genome association and population-based linkage analyses. Am J Hum Genet 2007;81:559-575.

12 Sörös P, Stanton SG: On Variability and Genes: Inter-individual Differences in Auditory Brain Function. Front Hum Neurosci 2012;6:150.

13 von Stechow L, Ruiz-Aracama A, van de Water B, et al: Identification of cisplatin-regulated metabolic pathways in pluripotent stem cells. PLoS One 2013;8:e76476.

14 Khrunin AV, Khokhrin DV, Moisseev AA, et al: Pharmacogenomic assessment of cisplatin-based chemotherapy outcomes in ovarian cancer. Pharmacogenomics 2014;15:329-337.

15 van As JW, van den Berg H, van Dalen EC: Medical interventions for the prevention of platinum-induced hearing loss in children with cancer. Cochrane Database Syst Rev 2014;7:CD009219.

16 Park MS, De Leon M, Devarajan P: Cisplatin induces apoptosis in LLC-PK1 cells via activation of mitochondrial pathways. J Am Soc Nephrol 2002;13:858-865.

17 García-Berrocal JR, Nevado J, Ramírez-Camacho R, et al: The anticancer drug cisplatin induces an intrinsic apoptotic pathway inside the inner ear. Br J Pharmacol 2007;152:1012-1020.

18 Rybak LP, Whitworth CA: Ototoxicity: therapeutic opportunities. Drug Discov Today 2005;10:1313-1321.

19 Campbell KC, Meech RP, Klemens Jj, et al: Prevention of noise- and drug-induced hearing loss with D-methionine. Hear Res 2007;226:92-103.

20 Videhult $\mathrm{P}$, Laurell G, Wallin I, et al: Kinetics of Cisplatin and its monohydrated complex with sulfur-containing compounds designed for local otoprotective administration. Exp Biol Med 2006;231:1638-1645.

21 Marina N, Chang KW, Malogolowkin M, et al: Children's Oncology Group. Amifostine does not protect against the ototoxicity of high-dose cisplatin combined with etoposide and bleomycin in pediatric germ-cell tumors: a Children's Oncology Group study. Cancer 2005;104:841-847. 


\section{CHAPTER 8}

\section{A first step towards personalized medicine in osteosarcoma: Pharmacogenetics as predictive marker of outcome after chemotherapy based treatment}

Melanie M. Hagleitner

Marieke J.H. Coenen

Hans J. Gelderblom

Remco R. Makkinje

Hanneke I. Vos

Eveline S.J.M. de Bont

Winette T.A. van der Graaf
H.W.Bart Schreuder

Uta Flucke

Frank N. van Leeuwen

Peter M. Hoogerbrugge

Henk-Jan Guchelaar

D. Maroeska W.M. te Loo 


\section{Abstract}

\section{Background}

Overall survival in patients with osteosarcoma is only $60 \%$. Poor response to chemotherapy is the dominant risk factor for poor survival. Pharmacogenetic research can offer possibilities to optimize treatment and improve outcome. We applied a pathway-based approach to evaluate the cumulative effect of genes involved in the metabolism of cisplatin and doxorubicin in relationship to clinical outcome.

\section{Methods}

In the discovery group we included 126 patients with osteosarcoma. To comprehensively assess common genetic variation in the 54 genes selected, linkage disequilibrium (LD, $r^{2}=0.8$ ) based tag-single nucleotide polymorphisms (SNP) strategy was used. A final set of 384 SNPs was typed using Illumina Beadarray platform. SNPs significantly associated with 5-year progression free survival (PFS) were replicated in another 64 patients with osteosarcoma.

\section{Results}

We identified five variants in FasL, MSH2, CASP3, ABCC5 and CYP3A4 that were associated with 5 -year PFS. Risk stratification based on the combined effects of the risk alleles showed a significant improvement of 5 -year PFS. Patients that carried no or only one risk allele had a 5 -year PFS of $100 \%$ compared to a 5 -year PFS of $84.4 \%$ for carriers of 2 or 3 risk alleles, $66.7 \%$ PFS if a patient carried $4-5$ alleles and a 5 -years PFS of $41.8 \%$ for patients with $>5$ risk alleles $(p<0.001)$

\section{Conclusion}

We identified several genes that showed association with PFS in patients with osteosarcoma. These pharmacogenetic risk factors might be useful to predict treatment outcome and to stratify patients immediately after diagnosis and offer the possibility to improve treatment and outcome.

\section{Introduction}

In the past decades, progression free survival (PFS) rates of patients with osteosarcoma have reached a plateau ${ }^{1}$. This is in contrast to other forms of cancer, where clear progress has been made, either by the use of new drugs or the ability of risk stratification and subsequently treatment adjustments ${ }^{2}$. Before the introduction of multiagent chemotherapy in the 1970s, when surgery was the only treatment, approximately $20 \%$ of the osteosarcoma patients survived ${ }^{3}$. Nowadays, cure rate for non-metastatic osteosarcoma patients approaches $55-65 \%{ }^{4}$. Several factors influence the outcome of patients with osteosarcoma. While factors such as site and size of the tumor, age at diagnosis, surgical approach and response of the tumor to pre-operative chemotherapy were shown to be important, only metastatic status at diagnosis is a consistent negative prognostic factor ${ }^{5,6}$. The absence of more reliable predictive factors at diagnosis has resulted in uniform chemotherapy schedules for all osteosarcoma patients. Being able to predict individua treatment response before start of treatment is however crucial to define new or alternative strategies in order to improve outcome.

Pharmacogenetics might help deliver this a priori knowledge, as has been shown for patients with colorectal cancer, acute lymphoblastic leukemia and other cancer types ${ }^{7,8}$. Although a number of studies have been performed into the role of genetic variants associated with the response to treatment in osteosarcoma patients ${ }^{9-11}$, none of these studies involved a comprehensive list of genes involved in the metabolism of cisplatin and doxorubicin, the two main drugs used in treatment of osteosarcoma patients. Hence, we investigated a set of genetic variants in 54 genes known to be involved in the metabolism of these drugs using a pathway-based genetic analysis. Subsequently, the role of the genetic variants in relationship to histological response and PFS was analyzed, demonstrating that it may be possible to define high risk groups for relapse on the basis of genetic variations in drug metabolizing pathways. 


\section{Patients and Methods}

\section{Study design}

We used a two-stage design. In the discovery stage, we genotyped single nucleotide polymorphisms (SNPs) in genes that encode drug-metabolizing enzymes in the cisplatin and doxorubicin pathway and investigated if these variants were associated with histologic response to neoadjuvant chemotherapy as well as 5-year PFS. In the second stage, SNPS demonstrating evidence of association in stage one were genotyped in an independent replication cohort subsequently a meta-analysis of the two datasets was performed.

\section{Patients}

In the discovery group, 126 patients with osteosarcoma treated in two different centers (Radboud university medical center and University Medical Center Groningen, The Netherlands) were included. The replication cohort consisted of 64 osteosarcoma patients treated at Leiden University Medical Center, The Netherlands. All patients were newly diagnosed, high-grade osteosarcoma with primary localization and/or metastatic disease and were consecutively treated between 1979 and 2008. The study was approved by the ethics committee of the Radboud university medical center as the central committee for this study. With respect to chemotherapy protocols, two different regimens were used. In one center of the discovery group (Radboud university medical center), patients diagnosed until 2002 were treated according to an institutional standard therapy consisting of a maximum cumulative cisplatin $\left(600 \mathrm{mg} / \mathrm{m}^{2}\right)$ and doxorubicine $\left(450 \mathrm{mg} / \mathrm{m}^{2}\right)$. After 2002 and in the other two centers (University Medical Center Groningen and Leiden University Medical Center) patients were treated according to the standard schedule as given in the EURAMOS study which basically consisted of cisplatin $\left(480 \mathrm{mg} / \mathrm{m}^{2}\right) /$ doxorubicin $\left(450 \mathrm{mg} / \mathrm{m}^{2}\right)$ and additionally high-dose MTX $\left(96-144 \mathrm{~g} / \mathrm{m}^{2}\right.$, according to age $)^{12}$. Clinical data were collected retrospectively.

In patients alive, DNA was extracted from blood using the QIAamp DNA Blood Midi kit (Qiagen, Venlo, The Netherlands) or saliva (Oragene saliva collection kit; DNA Genotek Kanata, Ontario, Canada) according to the protocol of the manufacturers. From patient who had died, DNA of paraffin-embedded samples was extracted as recently described ${ }^{13}$.

\section{Selection of SNPs and genotyping}

Literature (NCBI (PubMed, Gene)) and the Pharmacogenomics Knowledge Base (www.pharmgkb.org) were searched to select representative candidate genes involved in pathways relevant to cisplatin and doxorubicin. In the 54 selected genes we investigated SNPs that have been previously associated with functional changes. In addition, selected genes were covered with 5-10 tag SNPs to analyze the complete gene with a minimal set of SNPs. Tag SNP selection was performed in Haploview (LD statistic $r^{2}>=0.8$, minor allele frequency $>0.05$ ) using data from the HapMap project (International HapMap Consortium 2007, HapMap Data Rel24/phaseII Nov08). A final set of 381 SNPs was selected for analysis using the Illumina BeadarrayTM platform (Illumina, Inc., San Diego, CA). Illumina Genome Studio software was used for automated genotype clustering and calling. Samples and SNPs with genotyping call rates of $<85 \%$ were excluded from analyses, as well as SNPs deviating from Hardy-Weinberg equilibrium ( $p$-value $>0.05$ )

\section{Statistical analyses}

Statistical differences between the demographic data of the discovery and replication cohort were assessed by the Pearson-chi square test. Two-sided p-values are considered statistically significant if $<0.05$. No multiple testing correction has been applied due to the exploratory nature of the analysis. 5-year PFS was defined as the interval between diagnosis and disease progression/recurrence (=event) and was estimated using the Kaplan-Meier method. Patients without disease recurrence at the date of last follow-up were censored at that date. Histologic treatment response was defined as good responders with $<10 \%$ vital cells after pre-operative therapy with two cycles of each drug. Associations between potential confounders (gender, age at diagnosis, metastasis at diagnosis, axial location, MTX as co-medication and histologic response) and DFS or histologic response were evaluated using Cox's proportional hazard regression analysis or a $2 \times 2$ table, respectively. These statistical analyses were performed using SPSS version 20.0 (SPSS Inc., Chicago, III).

To assess the effect of a genetic variant on PFS and histologic response data were dichotomized in event/no event and good/bad responders, respectively. Multivariate logistic regression analyses of PFS and histologic response included MTX exposure (yes/no) in PLINK $^{14}$ using the command-logistic. A meta-analysis of the discovery and replication cohort was performed for the statistically significantly associated variants $(p<0.05)$ in the discovery cohort to investigate possible improved association signals. For SNPs with a low heterogeneity fixed effects $p$-values and for SNPs with a high heterogeneity the random effects $p$-value are reported.

To assess the effect of a combination of variants on PFS, a genetic risk score was composed as described in literature ${ }^{15}$. The score was constructed based on the number of unfavorable alleles $(0-1,2-3,4-5$ or $>5$ risk alleles) that were carried by each patient for each 
of the SNPs associated with PFS. With the risk alleles of each patient we analyzed 5-year PFS curves using the Kaplan-Meier method. The added value of the genetic risk score was evaluated to predict 5-year PFS in addition to metastases at diagnose.

\section{Results}

\section{Patients}

In total, we included 190 patients with newly diagnosed osteosarcoma. Patient characteristics are depicted in Table I. Baseline characteristics did not show statistically significant differences between the discovery and replication groups.

Table I. Demographic data

\begin{tabular}{|lcccc|}
\hline & Total $(\mathrm{N}=\mathbf{1 9 0})$ & Discovery $(\mathrm{N}=\mathbf{1 2 6})$ & Replication $(\mathrm{N}=64)$ & $\mathrm{p}$-value \\
Median age (years; median) & $16.9(2.8-67.3)$ & $15.6(2.8-39.5)$ & $18.2(5.0-67.3)$ & 0.5 \\
Male gender (N; \%) & $99(52.1 \%)$ & $67(53.2 \%)$ & $32(50 \%)$ & 0.68 \\
Primary metastases $(\mathrm{N} ; \%)$ & $39(20.5 \%)$ & $26(20.6 \%)$ & $13(20.3 \%)$ & 0.96 \\
Tumor in axial skeleton $(\mathrm{N} ; \%)$ & $13(6.8 \%)$ & $9(7.1 \%)$ & $4(6.2 \%)$ & 0.36 \\
Bad histologic response $(\mathrm{N} ; \%)$ & $112(66.3 \%)$ & $77(71.3 \%)$ & $35(57.4 \%)$ & 0.07 \\
5-year PFS & $60.50 \%$ & $57.90 \%$ & $65.60 \%$ & 0.43 \\
\hline
\end{tabular}

${ }^{1}$ comparing discovery vs. replication group

2patients available for analyse: Total cohort: $\mathrm{N}=115$. Disovery: $\mathrm{N}=73$. Replication: $\mathrm{N}=42$.

The number of patients with good histologic response was slightly higher in the replication group $(p=0.07)$. This might partially be explained by different treatment schedules as patients treated additionally with MTX had a slightly but not significantly better 5-year PFS $(66.7 \%$ vs. $54.5 \%, p=0.13)$. Achieving a good histologic response was not influenced by any of the baseline characteristics. No difference in 5-year PFS was seen comparing patients with good histologic response to bad responders $(61.3 \%$ vs. $55.8 \%, p=0.36)$. In contrast, 5-year PFS was significantly associated with co-treatment of high-dose MTX Therefore, MTX exposure was used as covariate in all multivariate analyses. No confounding factor was found for histologic response.

\section{Association analyses of histologic response}

In total, 177 samples (93.2\%) were genotyped successfully on the Illumina Bead Array system, 120 patients $(95.2 \%)$ in the discovery group and 57 patients $(89 \%)$ in the replication group (Figure I: flow chart)
Figure I. Flow chart of association analyses

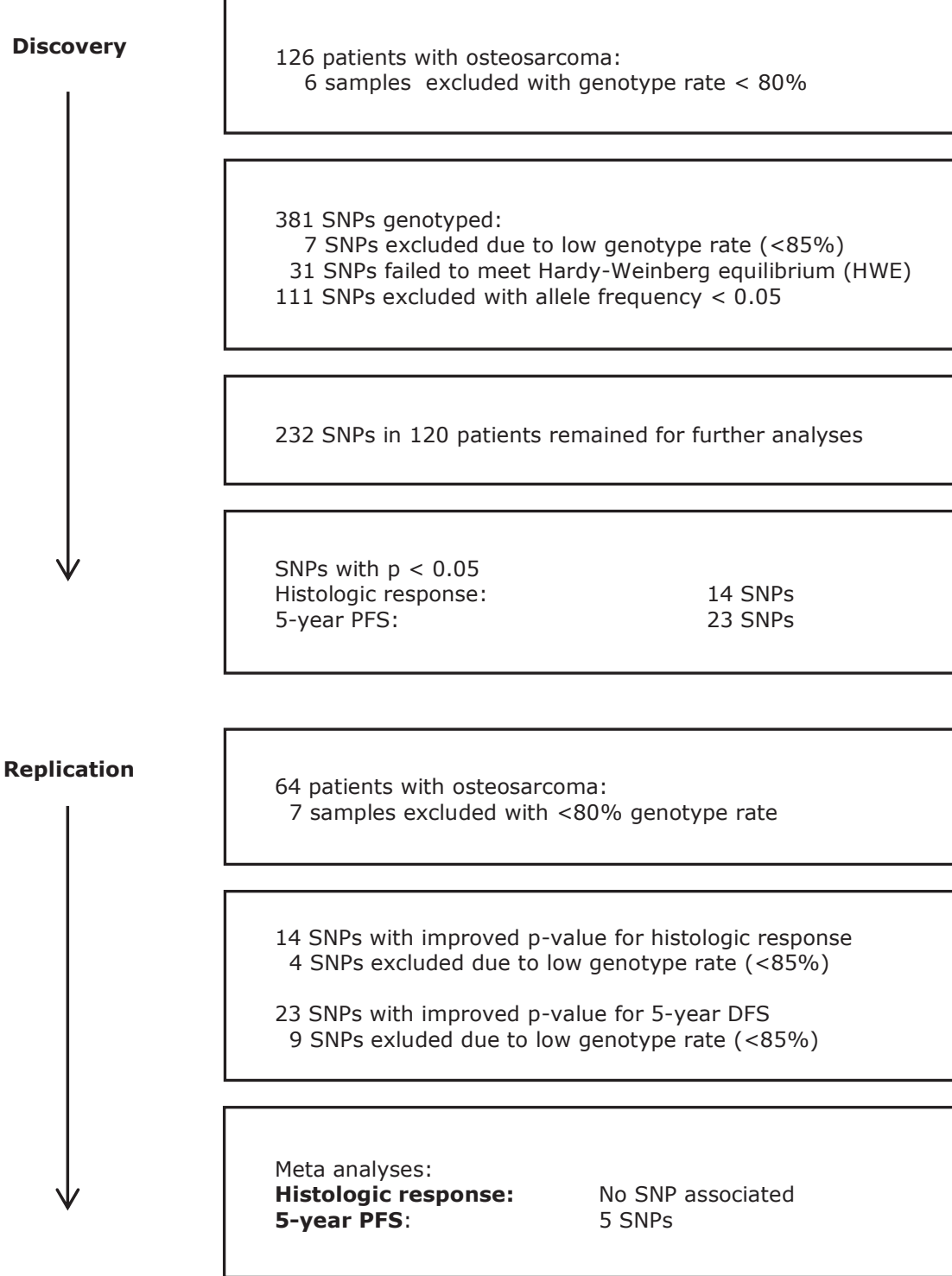

In the discovery group, 7 SNPs with genotyping call rates $<85 \%, 31$ SNPs deviating from Hardy-Weinberg equilibrium and 111 SNPs with an allele frequency $<0.05$ in the study population were excluded. In the discovery group, a total of 14 SNPs demonstrated a statistically significant association $(p<0.05)$ with histologic response (Supplementary Table I). Of these, four SNPs (rs11190291, rs723456, rs4645983, rs207455) were excluded because 
f low genotyping rates in the replication cohort. Meta-analyses of the remaining 10 SNPs, demonstrated 2 statistically significant associations (rs3136326 and rs1800936) however no improved association signal of the discovery and replication cohorts compared to the discovery cohort alone was seen (Supplementary Table I).

\section{Association analyses of 5-year PFS}

Multivariate linear regression analyses corrected for high-dose MTX resulted in the identification of 23 SNPs that demonstrated a statistically significant $(p<0.05)$ association with 5 -year PFS, in the discovery group. Of these 23 SNPs, nine SNPs (rs3181345, rs4647616, rs1233398, rs2808676, rs3176646, rs2917669, rs1805324, rs1296500, rs1895419) showed low call rates for the replication cohort, leaving 14 SNPs for analysis (Supplementary Table II). Five SNPs (rs763110, rs4638843, rs939338, rs2720376, rs4646437) demonstrated an improved association signal with PFS in the meta-analysis of the discovery and replication cohorts compared to the discovery cohort (Table II). These SNPs were not associated with histologic response.

Table II. Genetic variants associated with 5-years PFS

\begin{tabular}{|c|c|c|c|c|c|c|c|c|c|}
\hline \multirow[b]{2}{*}{ Gene } & \multirow[b]{2}{*}{ SNP } & \multirow[b]{2}{*}{ Allele } & & \multicolumn{3}{|c|}{ Discovery group } & \multicolumn{3}{|c|}{ Meta analyze fixed effects } \\
\hline & & & & $\mathbf{P}$ & OR & $95 \% \mathrm{Cl}$ & $\mathbf{P}$ & OR & $95 \% \mathrm{Cl}$ \\
\hline FasL & rs763110 & T & 5'UTR & 0.04 & 1.97 & $1.04-3.73$ & 0.02 & 1.76 & $1.08-2.87$ \\
\hline MSH2 & rs4638843 & c & Intron & 0.04 & 2.32 & $1.02-5.27$ & 0.007 & 2.66 & $1.31-5.36$ \\
\hline$A B C C 5$ & rs939338 & G & Intron & 0.03 & 1.86 & $1.06-3.24$ & 0.03 & 1.67 & $1.06-2.63$ \\
\hline CASP3 & rs2720376 & G & Intron & 0.02 & 0.52 & $0.30-0.90$ & 0.01 & 0.57 & $0.36-0.89$ \\
\hline СУРЗАА & rs4646437 & A & Intron & 0.02 & 0.34 & $0.13-0.85$ & 0.02 & 0.43 & $0.21-0.88$ \\
\hline
\end{tabular}

Multivariate linear regression analyses corrected for high-dose MTX

\section{Genetic risk score}

Our genetic risk score for PFS was calculated based on the five statistically significantly associated SNPs (rs763110, rs4638843, rs939338, rs2720376, rs4646437) in the meta-analysis of this study. Thus, the potential genotype score for each patient could range between 0 and 10. Since only a small number of patients had a score of 6 or higher, we merged these groups into one group for analysis, resulting into a total of four groups for the final analyses representing $0-1,2-3,4-5$ or more than 5 unfavorable alleles.

After adjusting for treatment with MTX, which was the only confounding factor for PFS in this study-group, we found that the genetic risk score was associated with 5-year PFS Patients without or one risk allele showed a survival of $100 \%$ compared to a 5-year PFS of $84.4 \%$ with $2-3$ risk alleles, $66.7 \%$ with $4-5$ alleles and $41.8 \%$ with $>5$ risk alleles $(p<0.001$, Figure II)
Figure II. 5-year PFS based on genetic risk score

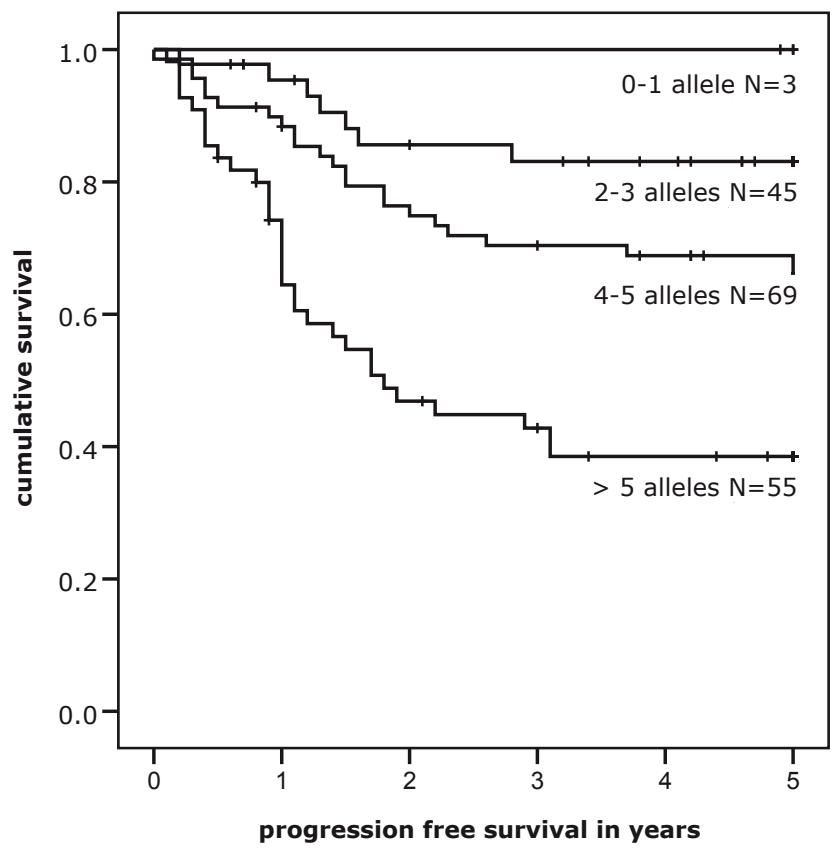

Adding the genetic risk score to the presence of metastases at diagnosis, the most important conventional risk factor, significantly improved the ability to predict PFS. The 5-year PFS of patients without metastases at diagnosis showed a similar effect with a surviva of $100 \%$ in patients carrying no or one risk allele compared to a 5 -year PFS of $85 \%$ in patients with 2-3 risk alleles, $69.8 \%$ with $4-5$ risk alleles and $53.8 \%$ in patients with $>5$ risk alleles $(p=0.008)$. In patients with metastases at diagnosis, all patients were carriers of at least 2 risk alleles, meaning there were no patients with no or one risk allele in this subgroup. The 5-year PFS of patients with 2-3 risk alleles and metastases at diagnosis was still $80 \%$. However, survival decreased to $56.2 \%$ in patients carrying $4-5$ risk alleles and only $12.5 \%$ in patients with $>5$ risk alleles $(p=0.007$; Figure III $)$ 
Figure III. 5-year PFS based on genetic risk score, analysis split based on the presence of metastases at diagnosis
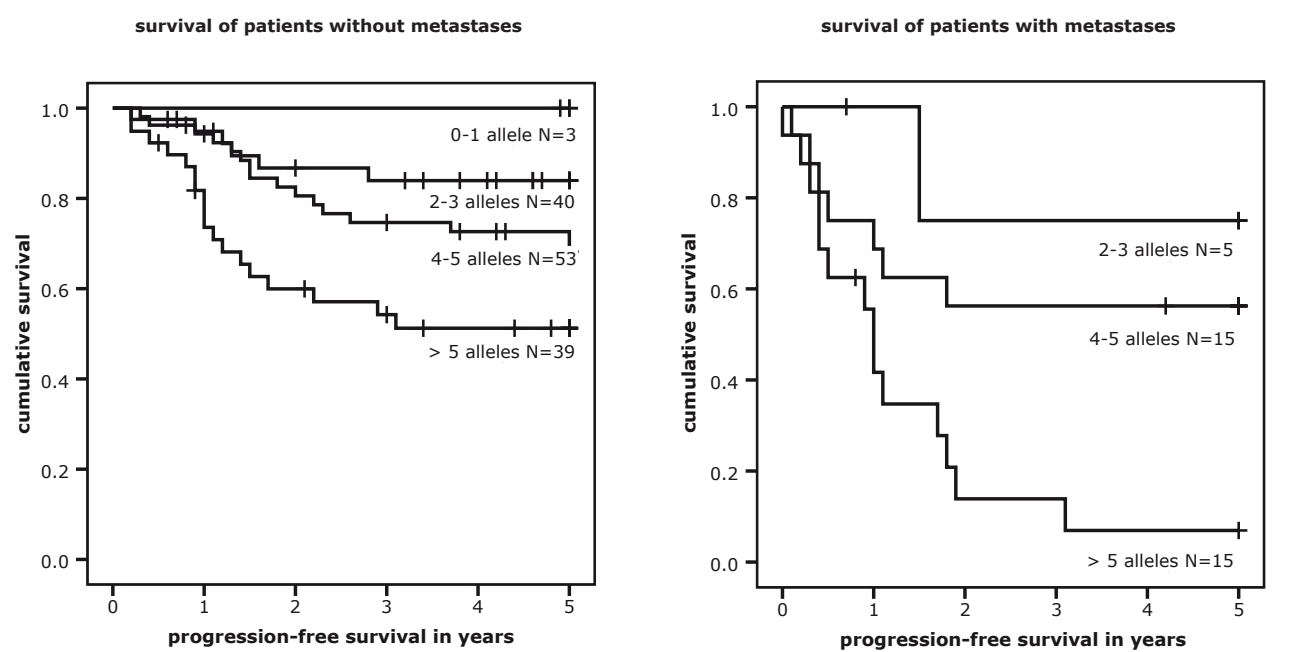

\section{Discussion}

In this study we showed that the pharmacogenetic profiles of patients with osteosarcoma may be used to stratify patients into good and bad responders. Based on our analysis five genetic variants were significantly associated with 5 -year PFS. To our knowledge this is the first pharmacogenetic study in osteosarcoma patients that demonstrate that it is possible to perform risk stratification at start of treatment for this specific patient group. Interestingly, histological response which is still the predominant factor for survival in many studies was not associated with 5-year PFS in this study. We are not the first to indicate that the value of histological response for treatment stratification might be limited. Several study trails have shown that intensification of chemotherapy preoperative resulted in a significant higher proportion of good responders, but outcome was similar ${ }^{16}$. Nevertheless, we investigated whether germline genetic polymorphisms can predict histologic response to pre-operative treatment. Although some associations where found in the discovery group, none of the identified SNPs showed an improved signal in the meta-analysis of both cohorts. Two of the associated SNPs remained significant though with higher p-values compared to the discovery cohort. It cannot be excluded that these SNPs represent true associations as our replication cohort is relatively small. Based on the preliminary results we advise that our findings should be validated in a larger cohort.

We identified one functional SNP in the Fas Ligand (FasL) gene and four tag-SNPs in the MutS homologue 2 (MSH2); Caspase 3 (CASP3); of the ATP-binding cassette, sub-familiy member 5 (ABCC5) and Cytochrome P450 3A4 (CYP3A4) to be associated with PFS. The minor allele of the variants in FasL, MSH2 and ABCC5 were associated with a lower survival, the minor allele of the other two SNPs were associated with a better survival (Supplementary Table II).

FasL induces apoptosis and appears to have a role in the development and progression of cancer, such as breast cancer ${ }^{17}$. The associated SNP, rs763110 (844T>C) of the FasL gene, is suggested to influence FASL expression ${ }^{18}$. It has been demonstrated that Fas expression correlates inversely with metastatic potential in human osteosarcoma cells. ${ }^{19}$ This is in accordance with our findings where the T allele, linked to a low expression of FasL, is associated with unfavorable disease outcome.

Contradictory results are shown with respect to the variant rs 4638843 in the MSH2 gene. In our study this variant was negatively associated with PFS while the same variant was shown to be positively associated with survival in colorectal cancer ${ }^{20}$. The $M S H 2$ gene encodes for the MSH2 protein which is part of the MutSa complex in the Mismatch Repair (MMR) system. This system recognizes specific types of DNA damage, as caused by cisplatin

Another pharmacogenetic association found in our study represents a variant in CASP3 which is one of the main executor genes in cell apoptosis. Association studies are scarce but, as CASP3 is at the heart of the apoptotic process it is reasonable to hypothesize that genetic variations in this gene have an influence on outcome.

In the present study we also identified a variant in $A B C C 5$ to be associated with an unfavorable 5-year PFS. It has been demonstrated that elevated expression of $A B C C 5$ (or MRP5) confers resistance to cisplatin and doxorubicin in different cell lines ${ }^{21}$. Additionally, it was shown that $A B C C 5$ supports osteoclast formation promoting the occurrence of breast cancer metastasis to the bone ${ }^{22}$.

The last association identified was with a variant in CYP3A4 which is involved in oxidation of cisplatin but also plays a role in the doxorubicin pathway. In osteosarcoma tumors, expression of CYP3A4 was found to be significantly higher in the primary tumor of patients who developed distant metastatic disease compared to patients with progression free survival ${ }^{23}$.

This pharmacogenetic analysis is the first in literature to report on a combined effect of genetic variants using a genotype risk score in osteosarcoma patients. To evaluate our findings we added conventional risk factors, such as metastases at diagnosis to the current SNP panel. Interestingly, in both patients with or without metastases at diagnosis we were able to distinguish patients with good outcome from patients with poor outcome based on the genetic risk score. The concept of combining metastatic status and genetic profile may be used to refine future protocols. We were able to identify a group of patients with very poor outcome which may benefit from alternative or novel treatment strategies. Our findings could have important implications for treatment and management of osteosarcoma and deserve validation in a larger, prospective cohort. Although the results of our study are promising, 
the number of patients included in these analyses is rather limited. Osteosarcoma is a rare disease and, at present, no other pharmacogenetic multidrug candidate pathway gene study in literature is available that includes a discovery and replication cohort as large as ours $(n=177)$. Including this study, a total of four outcome-related pharmacogenetic studies in patients with osteosarcoma have been published ${ }^{9-11}$. However, different treatment protocols including additional agents besides cisplatin and doxorubicin resulted in different strategies to select genes for pharmacogenetic analyses. As a consequence, most of the previously reported genes were not included in this study.

In conclusion, we identified several genes that might be associated with treatment response in patients with osteosarcoma. Replication of our data in additional cohorts and prospective clinical studies is warranted to reaffirm the potential of using pharmacogenetics in patients with osteosarcoma and bring about a more personalized treatment for these patients

\section{References}

Hagleitner MM, de Bont ES, Te Loo DM. Survival trends and long-term toxicity in pediatric patients with
osteosarcoma, Sarcoma 2012:2012:636r05.

2 Pui CH, Mullighan CG, Evans WE, et al. Pediatric acute lymphoblastic leukemia: where are we going and how
do we get there? Blood, 2012;120:1165-1174. Carter SKMD. The dilemma of adjuvant chemotherapy for osteogenic sarcoma. Cancer Clinical Trials.
$1980 ; 3: 29-36$.

4 Whelan JS, Jinks RC, McTiernan A, et al. Survival from high-grade localised extremity osteosarcoma: combined results and prognostic factors from three Europ
trials. Annals of Oncology. 2012;23:1607-1616.

5 Bielack SS, Kempf-Bielack B, Delling $G$, et al. Prognostic factors in high-grade osteosarcoma of the extremties or rrunk: an analysis of 1,702 patients treated on neoadjuvant cooperative osteosarcoma study grou

$6 \begin{aligned} & \text { Hagleitner MM, Hoogerbrugge PM, van der Graaf WT, et al. Age as prognostic factor in patients with osteo- } \\ & \text { sarcoma. Bone. 2011:49:1173-1177. }\end{aligned}$ 7 Toffoli $G$, Cecchin $E$, Corona $G$, et al. The role of UGT1A1*28 polymorphism in the pharmacodynamics and pharm.
3068 .

8 Schmiegelow $\mathrm{K}$, Forestier $\mathrm{E}$, Kristinsson J, et al. Thiopurine methyltransferase activity is related to the risk of relapse of childhood acute lymphoblastic leukemia: results from the NOPHO ALL-92 study. Leukemia.
$2009: 23: 557-564$.

Caronia D, Patino-Garcia A, Mine RL, et al. Common variations in ERCC2 are associated with response to cis-
platin chemotherapy and clinical outcome in osteosarcoma patients. Pharmacogenomics J. 2009:9: 347-353.

10 Caronia D, Patino-Garcia A, Perez-Martinez A, et al. Osteosarcoma Survival after Chemotherapy: A Pharma-

11 Windsor RE, Strauss SJ, Kallis $\mathrm{C}$, et al. Germline genetic polymorphisms may influence chemotherapy re-
sponse and disease outcome in osteosarcoma. Cancer. 2012;118:1856-1867.

12 Whelan J, Seddon B, Perisoglou M. Management of osteosarcoma. Curr Treat Options Oncol. 2006; 7: 444-

Hagleitner MM, Coenen MJ, Jeuken JW, et al. Taqman genotyping assays can be used on decalcified and par-
affin-embedded tissue from patients with osteosarcoma. Pediatr Blood Cancer. 2011;56:35-38.

14 Shaun Purcell, Benjamin Neale, Kathe Todd-Brown, et al. PLINK: A tool set for whole-genome association and

15 Kathiresan S, Melander O, Anevski D, et al. Polymorphisms associated with cholesterol and risk of cardiovas-

Anninga JK, Gelderblom H, Fiocco $\mathrm{M}$, et al. Chemotherapeutic adjuvant treatment for osteosarcoma: where
do we stand? Eur J Cancer. 2011; $47: 2431-2445$.

17 Mottolese M, Buglioni S, Bracalenti C, et al. Prognostic relevance of altered Fas (CD95)-system in human
breast cancer. Int J Cancer. 2000;89:127-132.

8 Wu J, Metz C, Xu X, et al. A novel polymorphic CAAT/enhancer-binding protein beta element in the FasL gene promoter alters Fas ligand expression: a candidate backgro
erythematosus patients. J Immunol. 2003;170:132-138.

19 Gordon N, Kleinerman ES. The role of Fas/FasL in the metastatic potential of osteosarcoma and targeting this
pathway for the treatment of osteosarcoma lung metastases. Cancer Treat Res. 2009;152:497-508.

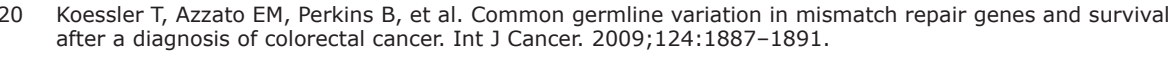

21 Pratt S, Shepard RL, Kandasamy RA, et al. The multidrug resistance protein 5 ( $(A B C C 5$ ) confers resistance to
5 -fluorouracil and transports its monophosphorylated metabolites. Mol Cancer Ther. 2005;4:855-863.

22 Mourskaia AA, Amir E, Dong Z, et al. ABCCC5 supports osteoclast formation and promotes breast cancer

metastasis to 23 Dhaini HR, Thomas DG, Giordano TJ, et al. Cytochrome P450 CYP3A4/5 Expression as a Biomarker of Out-
come in Osteosarcoma. Journal of Clinical Oncology. 2003:21:2481-2485. 



\section{CHAPTER 9}

\section{General discussion}




\section{Clinical relevance, general discussion and future perspectives}

Osteosarcoma is the most common primary malignant bone tumor in children and ad olescents. Although outcome improved after the introduction of multi-agent chemotherapy, survival for these patients is still poor. It appears that we have reached the limit in the survival of osteosarcoma patients achievable with currently available chemotherapy. The studies performed and described in this thesis were initiated to study the role of pharmacogenetics in the treatment of patients with osteosarcoma.

Since the 1970's survival for patients with osteosarcoma without metastatic disease at presentation, remains about $60 \%-65 \%{ }^{1}$. In case of detectable metastases at diagnosis overall survival is only $30 \%{ }^{2}$. Thus, new approaches in first line treatment are needed to improve survival. Despite new diagnostic and imaging techniques over the past view years and considerable improvements in surgical techniques that achieve local control with limp-sparing procedures, this has not resulted in improved overall survival. In addition, very little changes have been seen as far as systemic treatment concerns. Drugs such as interferon ${ }^{3}$ and monoclonal antibodies ${ }^{4}$ have been trialed in preclinical and clinical studies but showed no improvement in event free survival.

With the advances in sequencing technologies such as used in pharmacogenetic studies, metabolic pathways which can affect individual responses to drugs, can be studied. A better knowledge of the mechanisms responsible for response to current chemotherapeutic regimens may lead to the development of more effective approaches in the treatment of patients with osteosarcoma. Pharmacogenetic research studies the influence of inherited genetic variants associated with treatment response. These genetic variants can be evaluated for their pharmacokinetic and pharmacodynamic effects on relevant drugs in individual patients. One of the classical models concerning pharmacogenetics in oncology is the implementation of thiopurine methyltransferase (TPMT) genotype tests in the treatment of several non-malignant and malignant disorders with thiopurines such as mercaptopurine $^{5}$. Genetic variability in the TPMT gene has been associated with differing levels of TPMT leading to dose adaptations of mercaptopurine ${ }^{6}$. Similarly, a dose recommendation is available for the treatment of patients with 5 -fluorouracil as dihydropyrimidine dehydrogenase $(D P Y D)$ deficiency has been linked to sever toxicity?. The perception that part of this interpatient variability is inherited, and therefore predictable, may be applied in the development of personalized medicine. If pharmacogenetic research contributes to a better understanding of the mechanism of drug response in these patients, it may offer possibilities to individualize treatment and improve outcome and perhaps, even reduce toxicities.
Retrospective analysis of our cohort of patients with osteosarcoma showed that age was a predictive factor for overall survival

Further improvements in outcome of osteosarcoma patients may depend on the development of new agents and the refinement of the osteosarcoma therapy. Therefore, it may be helpful to identify biomarkers to differentiate patients into groups that will benefit more form a particular medicine than others. Beside the presence of metastasis at diagnose, there is no clear consensus regarding upfront relevant prognostic factors. In several malignancies age is a well-known prognostic factor and used for risk stratification. Its relevance in the treatment of osteosarcoma patients is however controversial. In pharmacological terms, age is a very interesting factor as age-related changes in the cell (e.g. oxidative stress, mitochondrial dysfunction, and various genetic mechanisms) are known to affect pharmacological responses ${ }^{8}$. Expression changes in enzymes have not only been seen in the first year of life but throughout childhood and adolescence ${ }^{9}$. Therefore, we retrospectively analyzed the role of age in the treatment of osteosarcoma patients as described in chapter 2. Beside the presence of metastases we showed that age was an independent prognostic factor for overall survival. Patients older than 20 years of age at time of diagnose had a poorer prognosis with an overall survival of $33.3 \%$ compared to young patients with overall survival of $70.6 \%$. Age remained a significant prognostic factor independent on metastatic stage at diagnosis and tumor location. After our data were published in 2011, the Children's Oncology Group (COG) described the influence of age on outcome in osteosarcoma patients in a large cohort of their four recent prospective North American Cooperative Group trials, including 1054 patients. Similar to our results, they showed that an age above 18 years was independent of other risk factors associated with poorer event free survival and overall survival and herewith confirmed our findings ${ }^{10}$. Similar to previous reports, this study found pelvic site, the presence of metastatic disease at diagnosis, positive tumor margins and a poor histologic response to chemotherapy to be significant predictors of poor prognosis.

Although, it is generally known that children are not little adults in many ways, the relevance of age-dependent changes in drug metabolizing, the so called developmental pharmacology ${ }^{11}$, has only recently been integrated into clinical decision making regarding drug doses. Age-related changes in body composition as well as growth and development of organs like liver, kidneys and intestine influence the absorption, distribution, metabolism and elimination of medications throughout infancy, childhood and adolescence ${ }^{11}$. So far, studies regarding developmental pharmacology are scars and predominantly focused on phase I enzymes such as the P-450 cytochromes (CYPs). However, evidence has shown that expression levels of several isoenzymes of the CYP family ${ }^{12}$ as well as hepatic and intestinal drug transporters ${ }^{9}$ vary throughout childhood and as such may influence pharmacokinetic of metabolized agents by these systems. For instance, the CYP3A4 expression levels ex- 
ceed adult levels in the first years of infancy ${ }^{12}$ e.g. the clearance of carbamazepine from plasma, which is metabolized by CYP3A4, is greater in children compared to adults ${ }^{13}$. This example shows that factors that cause variation in drug responses are complex involving genetic, environmental and physiological factors, like age.

\section{Analysis of pediatric osteosarcoma patients showed no significant differences regarding treat-} ment strategies over the past three decades

In the Netherlands, approximately 35-45 new patients with osteosarcoma are diagnosed each year which makes this tumor a rare disease. For our genetic analyses we included patients treated up to 30 years ago. For possible confounding factors, we studied in chapter 3 survival trends in our pediatric population of 130 children who were treated between 1979 and 2008. We found an improved survival in the patients treated in the last decade which was unexplained. In fact, since the 1970's treatment trials contained the same three-drug backbone consisting of cisplatin, doxorubicin and high-dose MTX. Furthermore, our study showed no significant changes in drug doses or patients' characteristics in the past three decades. As the basic treatment options have hardly changed since the 1970 we suggest that the slight improvement which is seen in the last decade might be attributable to evolving factors such as better supportive care.

Since patients today still receive exactly the same drugs as they would have 30 years ago, we were able to enlarge our dataset for the pharmacogenetic analyses. However, even more importantly, our analysis illustrates the stagnation of treatment opportunities and therefore improvement in survival of patients with osteosarcoma.

\section{A new method was developed to use historical paraffin embedded material for pharmacogenetic studies}

For our pharmacogenetic studies, we included data from patients treated between 1979 and 2008 of which approximately $50 \%$ had already died. Obviously, from these patients freshly isolated DNA was not available, but archived tumor and surrounding normal tissue from these patients is still stored at our department. To be able to analyze DNA of patients alive and patients that died, e.g. to prevent biased analysis of missing patients, we developed a method to obtain germline DNA from archived formalin-fixed paraffin-embedded (FFPE) tissues of patients that have died as described in chapter 4 . Using this modified protocol, we were able to obtain adequate DNA in decalcified FFPE tissue suggesting that with this adaptation, DNA can be successully recovered from decalcified archiv patients that are still alive several years after diagnosis. We compared freshly isolated DNA from blood with DNA from archived material from the same patient. Our results indicated that $100 \%$ of samples $(\mathrm{N}=18)$ had complete concordance for all genetic variants examined and confirmed that the processing and storage of DNA is not affecting the results.

Applying this procedure provides a new opportunity to analyze samples that have been preserved on FFPE. However, there is concern that DNA derived from FFPE tissues might be of limited value as it may not adequately represent the genetic data obtained from germline DNA. With us, other studies have addressed this this issue ${ }^{14,15}$. These studies consistently show that concordance between germline and FFPE material is virtually $100 \%$. In cancers like osteosarcoma with an incidence of 3-4 new cases per million per year, prospective studies are often too time consuming. With isolation of DNA from historical material it is possible to undertake comprehensive studies in much larger cohorts which is essential for pharmacogenetic research. Furthermore, many current clinical trials and hopefully all future clinical trials will archive patient's molecular information making these data accessible for future generations of genetic research.

\section{Candidate-gene analysis: Genetic variants may influence methotrexate plasma concentrations in pediatric malignancies}

In chapter 5, we performed a candidate-gene analysis to study the association of genetic variants with the elimination of MTX. The results of our study of 41 variants in 20 MTX-related genes showed two polymorphisms, ABCC2 3972C $>$ T and AMPD1 c.34C $>$ T, to be suggestively associated with an increased elimination of MTX 48 hours after infusion and a lower risk to develop high grade toxicity. A recently published study in 151 patients with ALL confirmed our findings regarding ABCC2 3972C >T. Lopez-Lopez and colleagues showed several SNPs in the $A B C C 2$ and $A B C C 4$ gene to be statistical significantly associated with MTX clearance and different toxicity parameters ${ }^{16}$. Patients with the CT/T genotype of the $A B C C 23972 C>T$ polymorphism showed lower MTX plasma concentrations at 72 hours after infusion compared to the CC genotype (MTX concentration $<0.2$ umol/I: $71.6 \%$ of CT/TT genotype vs. $50 \%$ of CC genotype; OR=0.40, $95 \% \mathrm{CI}: 0.18-0.86$, $\mathrm{p}=0.018$ ). Furthermore, a haplotype in $A B C C 2$ including the $A B C C 23972 \mathrm{C}>\mathrm{T}$ polymorphism showed the same effect on MTX toxicity as in our study. Therefore, the $A B C C 2$ polymorphism seems to play a role in the metabolism of MTX.

Several choices have been made regarding the method of this exploratory study. First, we focused on candidate genes and polymorphisms that are likely to have a direct impact on the function of the gene as to increase our power to identify relevan variants. Second, we optimized our power by including all $t=48$ hours MTX plasma levels after each course of each patient whereas others included only the highest score during 
the whole treatment ${ }^{17,18}$. Finally, we gained power by including ALL patients who were also treated with high-dose MTX according to the ALL-10 protocol ${ }^{19}$ at our department.

After the analyses of our data in 2008, a GWAS studying 398,699 SNPs has been published ${ }^{20}$. This study of over 400 patients with ALL reported associations with genetic variants in the anion transporter polypeptide, SLCO1B1. This transporter has not previously been associated with the MTX metabolism and therefore not selected for our own study. In this GWAS study, the strongest association was observed with the SLCO1B1 rs4149056 SNP. Recently, these results were replicated in two independent cohorts ${ }^{21,22}$. In both replication studies the variant rs4149056 was confirmed to be significantly associated with MTX kinetics. These subsequent findings illustrate the shortcomings of a candidate gene approach as was used in our study with potentially inadequate pre-selection of candidate genes. Nevertheless, our results can provide an incentive for future studies into pharmacogenetics of MTX in cancer, especially since identical results of the $A B C C 2$ variant have been seen in the recently published study of Lopez-Lopez and colleagues ${ }^{16}$.

Meta-analysis: The role of the MTHFR $677 \mathrm{C}>\mathrm{T}$ variant in methotrexate-induced liver toxicity in patients with cancer

The most widely studied genetic variant with respect to MTX metabolism is the MTHFR $677 \mathrm{C}>$ T variant as it is a key enzyme for intracellular folate homeostasis and metabolism. However, contradicting results for association with MTX-induced hepatic toxicity have been published ${ }^{17,18,23,24}$. Analogous to published data, our univariate analyses in chapter 6 showed indecisive results regarding the association between the variant in MTHFR and hepatic toxicity. Although, patients with the $677 \pi T$ genotype showed a clear trend for an increased risk to develop hepatotoxicity, results were not statistically significant. On the basis of the analysis of our data on 98 MTX-treated pediatric cancer patients and the meta-analysis, we concluded that in patients with cancer, the MTHFR 677T allele has a minor role in the development of MTX-induced hepatotoxicity.

By the time we published our results two other research groups had also investigated the influence of the MTHFR gene on toxicity in cancer patients by performing a meta-analysis. One study showed a positive association between the $677 \mathrm{C}>\mathrm{T}$ variant and hepatotoxicity 25 but similar to our results this was not confirmed in the second study by Lopez-Lopez and colleagues ${ }^{26}$. In our study we performed a number of subgroup analyses, in particular to determine whether therapy-related factors were associated to the development of hepatotoxicity. These subgroup analyses supported our overall findings of a minor role of the MTHFR 677 C $>$ T polymorphism on the development of hepatotoxicity.

\section{Our replication study failed to reproduce findings of an association between TPMT or COMT gene and ototoxicity}

In the study presented in chapter $\mathbf{7}$ we tried to replicate the promising data of Ross and colleagues who suggested an important role of TPMT and COMT in the development of cisplatin-induced ototoxicity ${ }^{27}$. Ross and colleagues identified patients at risk for cisplatin-induced ototoxicity and suggested that according to their genotype, these patients could be offered less toxic alternative therapy. Based solely on this specific study the US Food and Drug Administration has changed the cisplatin label to indicate the association of TPMT with ototoxicity ${ }^{28}$. These data were recently successfully replicated in an independent cohort by the same research group ${ }^{29}$. However, we could not replicate these data. Our findings are in concordance to the replication study of the St. Judes research group ${ }^{30}$ that also could not confirm the association between TPMT, COMT and ototoxicity. The results of our group and of the St Judes research group versus those of Ross and colleagues are contradictive. Differences between the initial study, our own study and the two replication studies may be caused by genetic but also non-genetic factors, such as different treatment regimens that can be highly variable in a clinical setting. We investigated a far more homogenous cohort compared to the initial study by Ross ${ }^{27}$, without potentially confounding factors such as cranial radiation and concomitant medication. Furthermore, the previously published studies were relatively low powered, with 162 patients in the initial study, 155 and 195 patients in respectively the two other replication studies. To address this issue further, we performed a meta-analysis of all published results on this topic. The variant rs4646361 in the COMT gene was the only association which remained statistically significantly associated with the development of cisplatin-induced ototoxicity, however the effect was much smaller compared to the initial study. It is debatable whether this association holds in a large-scale study. Herewith we show that the study of Ross and colleagues represents an illustrative example of the vulnerability of small studies to detect false-positive finding ${ }^{31}$. The initial study used a panel of nearly 2000 variations capturing 220 key drug metabolism genes. The association between TMPT and COMT with cisplatin-induced ototoxicity was unexpected. First, platinum-based products are no known substrates for TPMT and second, only a single study has implicated COMT in sensorineuronal deafness, however the molecular mechanism is still unclear ${ }^{32}$. Therefore, before considering clinical application of a genetic test, research should be performed in well powered cohorts of well-characterized phenotypes and routinely include a replication cohort for validation. 
Our pathway-gene association study shows first steps to personalized medicine in the treatment of patients with osteosarcoma

The pharmacogenetic study described in chapter $\mathbf{8}$ investigates, to our knowledge, the most comprehensive set of SNPs that are involved in the metabolism of cisplatin and doxorubicin in patients with osteosarcoma. This pathway-gene association study of $177 \mathrm{pa}$ tients, which is the largest cohort of patients with osteosarcoma for pharmacogenetic studies to date, showed two interesting findings. First, histologic response was not associated with a 5-year PFS. This finding is not completely unexpected as it has been suggested by others ${ }^{33,34}$. Growing evidence shows that the positive effect of a good histologic response regarding favorable outcome is dependent on the combination and dose of agents given in the neoadjuvant traject ${ }^{33}$. Moreover, histologic response cannot be assessed at the time of diagnose making this factor unattractive to predict survival. Second, we were the first and only study to develop a stratification score based on the associated genetic variants showing an additive effect of unfavorable variables. In the subgroup of patients with metastases at diagnose the genetic risk score distinguished patients with a relatively good outcome to the group of patients with poor survival. The presence of metastases at diagnose is the most consistent prognostic factor. The ability to differentiate patients with even a worse outcome in this subgroup gives new opportunities in treatment strategy. These patients may be candidates for participation in clinical trials of new agents.

Including our study, a total of four outcome-related pharmacogenetic studies in patients with osteosarcoma have been published. The research group of Caronia and colleagues 35,3 have performed two candidate gene studies identifying polymorphism in $E R C C 2, A B C C 3$ and $A B C B 1$ gene to be associated with osteosarcoma outcome. A pilot study of Windsor and colleagues $^{37}$ found an association of the CCNP1 gene with outcome. Of all associated genes in the different studies interestingly only one variant in the $A B C B 1$ gene (rs1128503) was studied by two research groups. This variant was positively associated with survival in the study of Caronia but showed no statistical significance in the study of Windsor and colleagues. Different study protocols with different combination of agents resulted in the variety of different genes that have been investigated so far. Hence, a pharmacogenetic profile for survival might as well be protocol-specific.

\section{Future perspectives}

It is clear from our studies and data from others, that the benefit of traditional chemotherapy containing cisplatin, doxorubicin and high-dose MTX has reached its limits. In particular, for patients with unfavorable outcome no alternative effective therapy is currently available. In the last five years, several clinical trials of investigational agents have been published. One of the most promising agents is the immunomodulator interferon-a (IFN a) which is shown to enhance the sensitivity of human osteosarcoma cells to agent such as doxorubicin by p53-dependent apoptosis ${ }^{38,39}$, and is incorporated in the phase III Euramos I trial. Patients with good histologic response to neoadjuvant therapy were randomized to continue traditional chemotherapy or receive additional IFN a. Preliminary data presented at ASCO 2013 congress $^{3}$ showed no differences in a rather short follow-up period of 3-year event free survival between the arms $(77 \%$ in the IFN a arm vs. $74 \%$ in the standard arm). It might be possible that a longer follow-up period will show more effect of IFN a as the median time to relapse is approximately 3-4 years after end of treatment. Anothe immunomodulator being studied in phase II trials is muramyl tripeptide-phophatidyl ethanolamine (MTP-PE). First results showed improved overall survival in both patients with local disease and patients with metastatic disease ${ }^{40}$. Regarding bisphosphonates, positive results in several models of osteosarcoma cell lines lead to phase I/II trials of the bisphosphonate, zoledronate. Phase III trials on this agent have still to be performed. Another increasingly important class of anticancer therapies are monoclonal antibodies. So far, trastuzumab which is a monoclonal antibody to HER2 was administered in a phase II tria in patients whose osteosarcoma tumor overexpresses HER2. However, no statistical difference was found between the study arm and the standard arm ${ }^{41}$. There is a clear need for newer effective agents for patients with osteosarcoma.

In this thesis we used a candidate- or pathway gene approach which is a hypothesis driven method to investigate genetic variants in genes known to be associated with the trait of interest e.g. metabolism of the used medication. However, this approach is limited to the established pharmacological pathway. Not all genes relevant for the metabolism of the chemotherapeutic agents might have been studied. A more extensive study involving all genetic variants in the entire biologic pathway will therefore be a logical next step. Techniques such as the DMET (drug metabolism enzymes and transporters) enable specifically genetic analysis of the involvement of metabolic pathways in drug metabolism. It provides information concerning 1936 genetic variations in 225 genes involved in drug disposition. For identification of significant new biomarkers the DMET is therefore suited to understand more about the pharmacological response observed in patients. Another technique is a GWAS which is a good approach to study the entire genome in a hypothesis free manne to identify genes not previously known to be associated with a specific trait. Recently, an impressive study in the field of rheumatoid arthritis (RA) has performed a GWAS in more than 100.000 patients and identified target genes of approved therapies for RA and suggested drugs approved for other indications to be repurposed for the treatment of $\mathrm{RA}^{42}$. GWA studies aiming to identify predictive markers for drug efficacy end points such as progression-free survival however, necessitate large cohorts. Especially, when studying associations between clinical outcome and genetic polymorphisms large cohorts with well-controlled clinical protocols are required. These GWA studies can be performed only through large international collaborative efforts and with extensive biobanking. Therefore we recently indicated a comprehensive study investigating the influence of genetic poly- 
morphisms using patients randomized in EURAMOS-1. In total 450 participants will be included and studied to identify and replicate genetic associations in a complex disease like osteosarcoma ${ }^{43}$. Furthermore, GWAS studies detect association not causations. The only way to show causality is to understand the biological pathway and the role that the gene variant has in the production of the trait or disease process. Thus, the results of GWAS studies should always be considered in the light of functional evidence.

Before the start of this project, studies concerning the influence of genetic variants in the treatment of osteosarcoma disease were limited to some cases within the context of a bigger cohort of cancer patients. The stagnation of treatment opportunities and thereby improvement in survival of patients with osteosarcoma was the main reason to initiate the studies performed and described in this thesis.

Since the first studies regarding pharmacogenetics have been published, a large volume of data on many potentially clinically relevant genetic variants of drug efficacy and toxicity has been reported. Until recently, most data regard monogenetic studies or genes encoding drug-metabolizing enzymes. Only a handful of studies have found their way into clinica daily routine. More recently, advances in sequencing technologies have enabled broader, genome-wide searches for variants of drug response. In a remarkable number of pharmacogenomics reviews, authors widely speculated about the potential clinical benefits of pharmacogenomics in the clinical setting. However, it is clear that an increasingly complex series of barriers, such as the discovery of well-defined genotype-phenotype associations, reproducibility, biological function of genetic variants, etc. must be overcome before the implementation of pharmacogenomics in the clinical setting can be realized.

\section{References}

Link MP, Gebhardt MC, Meyers PA. Osteosarcoma. In: Pizzo PA, Poplack DG, editors. Principles and practice
of pediatric oncology. 2nd ed. Philadelphia: Lippincott Williams \& Wilkins; 2002. p.1502-1589. Bacci $G$, Picci P, Ferrari $S$, et al. Primary chemotherapy and delayed surgery for nonmetastatic osteosarcoma
of the extremities: Results in 164 patients preoperatively treated with high doses of methotrexate followed by

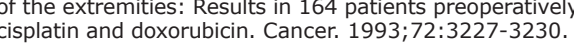

3 Bielack SS, Smeland S, Whelan J, et al. MAP plus maintenance pegylated interferon \{alpha\}-2b (MAP-IFN) versus MAP alone in patients (pts) with resectable high-grade osteosarcoma and good histologic response to preoperative MAP: :irst results 10
ABSTRACTS 2013:LBA10504

4 Ebb $\mathrm{D}$, Meyers $\mathrm{P}$, Grier $\mathrm{H}$, et al. Phase II trial of trastuzumab in combination with cytotoxic chemotherapy for treatment of metastatic osteosarcoma with human epidermal growth factor
report from the children's oncology group. 3 Clin Oncol. 2012; $30: 2545-2551$.

5 Relling MV, Gardner EE, Sandborn WJ, et al. Clinical Pharmacogenetics Implementation Consortium guide-
lines for thiopurine methyltransferase genotype and thiopurine dosing. Clin Pharmacol Ther. 2011;89:387-

6 Teml A, Schaeffeler E, Herrlinger KR, et al. Thiopurine treatment in inflammatory bowel disease: clinical
pharmacology and implication of pharmacogenetically guided dosing. Clin Pharmacokinet. 2007; $46: 187-208$, 7 Caudle KE, Thorn CF, Klein TE, et al. Clinical Pharmacogenetics Implementation Consortium guidelines for 645.

8 McLean AJ, Le Couteur DG. Aging biology and geriatric clinical pharmacology. Pharmacol Rev. 2004;56:163Mooij MG, Schwarz UI, de Koning BA, et al. Ontogeny of human hepatic and intestinal transporter gene
expression during childhood: age matters. Drug Metab Dispos. 2014;42:1268-1274.

10 Janeway KA, Barkauskas DA, Krailo MD, et al. Outcome for adolescent and young adult patients with osteo-
sarcoma: a report from the Children's Oncology Group. Cancer. 2012;118:4597-4605. Kearns GL, Abdel-Rahman $\mathrm{SM}$, Alander SW, et al. Developmental pharmacology-drug disposition, action, and
therapy in infants and children. $\mathrm{N} \mathrm{Engl} \mathrm{J}$ Med. 2003;349:1157-1167.

12 van den Anker JN. Developmental pharmacology. Dev Disabil Res Rev. 2010;16:233-238.

13 Riva $R$, Contin $M$, Albani F, et al. Free concentration of carbamazepine and carbamazepine-10,11-epoxide in
children and adults. Influence of age and phenobarbitone co-medication. Clin Pharmacokinet. 1985; 10:524531.

Xie B1, Freudenheim JL, Cummings SS et al. Accurate genotyping from paraffin-embedded normal tissue
adjacent to breast cancer. Carcinogenesis. 2006;27:307-310.

Marsh S, Mallon MA, Goodfellow P, et al. Concordance of pharmacogenetic markers in germline and colorectal
tumor DNA. Pharmacogenomics. 2005:6:873-877.

16 Lopez-Lopez E1, Ballesteros J, Piñan MA, et al. Polymorphisms in the methotrexate transport pathway: a new tool for MTX plasma level prediction in pediatric acute lymphoblastic leukemia. Pharmacogenet Genomics.

Imanishi $\mathrm{H}$, Okamura $\mathrm{N}$, Yagi $\mathrm{M}$, et al. Genetic polymorphisms associated with adverse events and elimination of methotrexate in
2007;52:166-171.

18 Chiusolo P, Giammarco $S$, Bellesi $S$, et al. The role of MTHFR and RFC1 polymorphisms on toxicity and outcome of adult patients with hematological malignancies treated with
leucovorin rescue. Cancer Chemother Pharmacol. 2012;69:691-696.

Kamps $W_{A}$, van der Pal-de Bruin KM, Veerman AJ, et al. Long-term results of Dutch Childhood Oncology
Group studies for children with acute lymphoblastic leukemia from 1984 to 2004. Leukemia 2010;24:309319.

Treviño $L R$, Shimasaki N, Yang $W$, et al. Germline genetic variation in an organic anion transporter polypep-
tide associated with methotrexate pharmacokinetics and clinical effects. J Clin Oncol. 2009; 27:5972-5978.

Radtke $\mathrm{S}$, Zolk $\mathrm{O}$, Renner $\mathrm{B}$, et al. Germline genetic variations in methotrexate candidate genes are as2013;121:5145-5153.

22 Ramsey $L B$, Panetta JC, Smith C, et al. Genome-wide study of methotrexate clearance replicates SLCO1B1.
Blood. 2013;121:898-904.

23 Aplenc $R$, Thompson $\mathrm{J}$, Han P, et al. Methylenetetrahydrofolate Reductase polymorphisms and therapy
response in pediatric acute lymphoblastic leukemia. Cancer Res 2005;65:2482-2487.

24 Seidemann K, Book M, Zimmermann M, et al. MTHFR 677 (C-T) polymorphism is not relevant for prognosis 
or therapy-associated toxicity in pediatric NHL: results from 484 patients of multicenter trial NHL-BFM 95. Ann Hematol 2006;85:291-300.

25 Yang L, Hu X, Xu L. Impact of methylenetetrahydrofolate reductase (MTHFR) polymorphisms on methotrexate-induced toxicities in acute lymphoblastic leukemia: a meta-analysis. Tumor Biol. 2012;33:1445-1454.

26 Lopez-Lopez E, Martin-Guerrero I, Ballesteros J, et al. A systematic review and meta-analysis of MTHFR polymorphisms in methotrexate toxicity prediction in pediatric acute lymphoblastic leukemia. Pharmacogenomics J. $2013 ; 13: 498-506$.

27 Ross CJ, Katzov-Eckert H, Dubé MP, et al. Genetic variants in TPMT and COMT are associated with hearing loss in children receiving cisplatin chemotherapy. Nat Genet. 2009;41:1345-1349.

28 Green D. Pharmacogenetic changes to the FDA-approved PLATINOL $®$ (cisplatin) label include safety update regarding cisplatin-induced hearing loss in children. PPAG Article feb 2012.

29 Pussegoda K, Ross CJ, Visscher H, et al: Replication of TPMT and ABCC3 genetic variants highly associated with cisplatin-induced hearing loss in children. Clin Pharmacol Ther. 2013; 94:243-251.

30 Yang JJ, Lim JY, Huang J, et al. The role of inherited TPMT and COMT genetic variation in cisplatin-induced ototoxicity in children with cancer. Clin Pharmacol Ther. 2013;94:252-259.

31 Johnson JA, Klein TE, Relling MV. Clinical implementation of pharmacogenetics: more than one gene at a time. Clin Pharmacol Ther. 2013;93:384-385.

32 Du X, Schwander M, Moresco EM, et al. A catechol-O-methyltransferase that is essential for auditory function in mice and humans. Proc Natl Acad Sci U S A. 2008;105:14609-14614.

33 Anninga JK, Gelderblom H, Fiocco M, et al. Chemotherapeutic adjuvant treatment for osteosarcoma: where do we stand? Eur J Cancer. 2011;47:2431-2445.

34 Lewis IJ, Nooij MA, Whelan J, et al. Improvement in histologic response but not survival in osteosarcoma patients treated with intensified chemotherapy: a randomized phase III trial of the European Osteosarcoma Intergroup. J Natl Cancer Inst. 2007;99:112-128.

35 Caronia D, Patino-Garcia A, Milne RL, et al. Common variations in ERCC2 are associated with response to cisplatin chemotherapy and clinical outcome in osteosarcoma patients. Pharmacogenomics J. 2009;9:347-353.

36 Caronia D, Patino-Garcia A, Perez-Martınez A, et al. Osteosarcoma Survival after Chemotherapy: A Pharmacogenetic Study. PLoS One 2011;6:e26091.

37 Windsor RE, Strauss SJ, Kallis C, et al. Germline genetic polymorphisms may influence chemotherapy response and disease outcome in osteosarcoma. Cancer. 2012;118:1856-1867.

38 Jia SF, An T, Worth L, et al. Interferon-alpha enhances the sensitivity of human osteosarcoma cells to etoposide. J Interferon Cytokine Res. 1999;19:617-624.

39 Yuan XW, Zhu XF, Huang XF, et al. Interferon-alpha enhances sensitivity of human osteosarcoma U2OS cells to doxorubicin by p53-dependent apoptosis. Acta Pharmacol Sin. 2007;28:1835-1841.

40 Meyers PA, Chou AJ. Muramyl Tripeptide-Phosphatidyl Ethanolamine Encapsulated in Liposomes (L-MTP-PE) in the Treatment of Osteosarcoma. Adv Exp Med Biol. 2014;804:307-321.

41 Ebb D, Meyers P, Grier H, et al. Phase II trial of trastuzumab in combination with cytotoxic chemotherapy for treatment of metastatic osteosarcoma with human epidermal growth factor receptor 2 overexpression: a report from the children's oncology group. J Clin Oncol. 2012;30:2545-2551.

42 Okada Y1, Wu D2, Trynka G1, et al. Genetics of rheumatoid arthritis contributes to biology and drug discovery. Nature. 2014;506:376-381

43 Burton PR1, Hansell AL, Fortier I. Size matters: just how big is BIG?: Quantifying realistic sample size requirements for human genome epidemiology. Int J Epidemiol. 2009;38:263-273. 


\section{Summary}


Osteosarcomas are the most frequent bone tumors in childhood and adolescence. Although disease-free survival improved by the introduction of chemotherapy in the late 1970's to approximately $60-70 \%$ in non-metastatic disease and $20-30 \%$ in metastatic disease, still $50 \%$ of the patients with osteosarcoma will not survive. A poor response to chemotherapy is the dominant risk factor for poor survival. A better knowledge of the mechanisms responsible for poor response and adverse events related to current chemotherapeutic regimens may lead to the development of more effective chemotherapeutic approaches and improve outcome and perhaps, even reduce toxicities. One way to improve treatmen of the patients is at the level of pharmacogenetics which refers to genetic differences af fecting individual responses to drugs, both in terms of therapeutic effect as well as adverse effects. In this thesis the relevance of polymorphisms in genes involved in the metabolism of chemotherapeutics used in the treatment of patients with osteosarcomas was studied.

Chapter 1 contains a general introduction and describes current strategies for treating patients with osteosarcoma, the relation between prognostic factors and survival and gives a short overview on the current knowledge concerning the role of pharmacogenetics in the treatment of cancer

Chapter 2 describes a retrospective analysis with the aim to analyze whether age has an influence on outcome. Osteosarcoma patients treated in our hospital between 1979 and 2008 were included in this study. In total, 102 patients with primary osteosarcoma were included. Overall survival (OS) of the whole population was $53.5 \%$ with a 5 -years disease-free survival (DSF) of $47.1 \%$. Beside known prognostic factors, such as histological response and the presence of metastases, age markedly affected survival with an OS of $70.6 \%$ in patients below 10 years of age, $52.5 \%$ in patients $11-19$ years and $33.3 \%$ in the patient group 20-40 years $(p=0.002)$. When analyzed as continuous variable, age was found to be inversely proportional related to survival $(p=0.048)$. In conclusion, younger patients have a significantly better outcome than older patients.

Chapter $\mathbf{3}$ focused on trends in survival rates and long-term toxicity in pediatric patients with osteosarcoma. In this study, we retrospectively evaluated, whether after the introduction of neoadjuvant chemotherapy in the late seventies, further improvement in outcome of osteosarcoma patients was achieved. In total, 130 pediatric patients under the age of 20 years with newly diagnosed, primary localized or metastatic high-grade osteosarcoma were analyzed regarding demographic, treatment-related variables, long-term toxicity and survival data. The overall period of 30 years was split into sub-periods relating to the major changes in treatment protocol (1979-1983, 1984-2002, and 2003-2008). Comparison of the different time periods showed that the 5 -year OS improved from $58.6 \%$ for children diagnosed during $1979-1983$ to $78.6 \%$ for those diagnosed during 2003-2008 $(p=0.13$ ). Interestingly, treatment agents and dosage basically remained the same over the past 30 years in this study. Therefore, other factors like empiric use of broad spectrum antibiotics in case of neutropenic fever, pain management, antiemetic's, nutrition, blood banking and improved pediatric intensive care might have improved the acceptance of the current treatment. Furthermore, we showed that late cardiac effects and second malignant neoplasms can become evident many years after treatment and can have profound impact on cancer survivors.

Chapter 4 describes the quality control of assays and technologies applied for the isolation of DNA from long-time stored, decalcified, paraffin-embedded tissue. In cancers like osteosarcoma with approximately $40-50 \%$ of patients who have died, archived histological specimens are often the only accessible source of biological material. However, this material has been decalcified and paraffin-embedded for many years which can severely affect the quality of the DNA. Therefore, we developed a method to extract high quality DNA from stored and decalcified, formalin-fixed paraffin-embedded tissues. Subsequent downstream analysis showed that the obtained DNA was of sufficient quality to be used for the methods described in this thesis. Our downstream analysis showed $100 \%$ concordance between genotypes obtained from DNA of FFPE tissue and the genotypes obtained from DNA of blood or saliva (the normal DNA source) from the same patients. In this chapter, we have demonstrated that formalin fixed and paraffin embedded tissue, whether decalcified or not, can be used for genetic polymorphism analysis using Taqman ${ }^{\circledR}$ allelic discrimination assays. This forms an unique opportunity to combine new insights in genetic research with historical patient cohorts.

In chapter $\mathbf{5}$ we analyzed genetic variants in 20 genes involved in the metabolic pathway of methotrexate (MTX) in patients with osteosarcoma for their association with delayed MTX elimination. High-dose methotrexate (MTX) forms a cornerstone in the treatment of several pediatric malignancies including osteosarcoma. Delayed MTX elimination increases the risk of treatment associated toxicity and necessitates increased leucovorin rescue and additional hospitalization for hydration and urinary alkalinization. In total, 640 courses of high-dose MTX were evaluated. At least one episode of MTX-induced toxicity grade 3 or 4 was recorded in $41.8 \%$ of the patients. Four polymorphisms showed an association with MTX plasma levels with p-value <0.10: ABCC2 c. 3972C > T, AMPD1 c.34C >T, ITPA c. 94C > T and GSTT1del. Association analyses for the four polymorphisms for MTX-induced toxicity (grade 3 and 4) showed however no statistically significant association. In conclusion, we found indications that pharmacogenetic variants affect MTX plasma levels. In contrast to the expectations the variants were not associated with toxicity. Nevertheless, our results can provide an incentive for future studies into pharmacogenetics of MTX in childhood cancer. 
In chapter 6 we investigated the role of the MTHFR c.677C>T polymorphism in MTX-induced hepatotoxicity by analyzing a Dutch cohort of pediatric patients treated with high doses of MTX and subsequently performing a meta-analysis. Our cohort contained 98 patients with osteosarcoma or ALL receiving 542 courses of high-dose MTX. Hepatotoxicity was evaluated retrospectively according to common terminology criteria for adverse events (National Cancer Institute Criteria). The analysis showed a 1.8-fold increased risk for the $T$ vs. the $C$ allele ( $95 \%$ CI $1.0-3.2, p=0.04$ ). This finding was not supported by the meta-analysis including seven studies and 1044 patients; the OR for the $677 \mathrm{~T}$ versus $\mathrm{C}$ allele was 1.1 (95\% CI: 0.84-1.5, $\mathrm{p}=0.25)$. In conclusion, in patients with cancer, the THFR 677T allele has only a minor role in the development of MTX-induced hepatotoxicity.

Chapter 7 aimed to evaluate the role of polymorphisms in the TPMT and COMT genes in cisplatin-induced ototoxicity as recent studies have suggested that variants in these genes can predict the development of cisplatin-induced hearing loss. Therefore, two independent cohorts of 110 Dutch and 38 Spanish patients with osteosarcoma were investigated and a meta-analysis was performed including all previously published studies resulting in a total population of 664 patients with cancer. Only, the genetic variant rs4646316 in the COMT gene showed a significant association with ototoxicity (OR A versus T allele $=1.5,95 \% \mathrm{CI}$ : 1.14-1.97, $p=0.003)$. However, the effect was much smaller than reported in previously published studies. With this largest meta-analysis performed to date, our study showed that the influence of TPMT and COMT on the development of cisplatin-induced hearing loss may be less important than previously suggested.

In chapter 8 we applied a pathway-based approach to evaluate the cumulative effect of genes involved in the metabolism of cisplatin and doxorubicin in relationship to clinical outcome. In the 54 selected genes we investigated SNPs that have previously associated with functional changes. For completeness, we covered the selected genes with tag SNPS to analyze the whole gene with a minimal set of SNPs. In the 177 patients, five variants were identified in FasL, MSH2, CASP3, ABCC5 and CYP3A4 to be associated with 5-year progression free survival (PFS). Risk stratification based on the combined effects of the risk alleles showed a significant improvement of 5-year PFS. Patients that carried no or only one risk allele had a 5 -year PFS of $100 \%$ compared to a 5 -year PFS of $84.4 \%$ for carriers of 2 or 3 risk alleles, $66.7 \%$ PFS if a patient carried $4-5$ alleles and a 5 -years PFS of $41.8 \%$ for patients with $>5$ risk alleles $(p<0.001)$. The pharmacogenetic risk factors identified in this study might be useful to predict treatment outcome and to stratify patients immediately after diagnosis and offer the possibility to improve treatment and outcome.

A discussion of the studies of this thesis and future research perspectives can be found in chapter 9. 


\section{Samenvatting}


Het osteosarcoom is de meest voorkomende bottumor op de kinder- en adolescentenleeftijd. Desondanks is het een niet vaak voorkomende ziekte met een incidentie van slechts 35-45 nieuwe gevallen per jaar in Nederland. Door de invoering van pre- en postoperatieve chemotherapie is de overleving van patiënten met een osteosarcoom sterk verbeterd van $20-30 \%$ in de jaren zeventig naar ongeveer $60-70 \%$ tegenwoordig. Echter ondanks deze duidelijke verbetering, reageert nog steeds een groot deel van de patiënten onvoldoende op de behandeling en overlijdt. De reden hiervoor heeft mogelijk te maken met de ongevoeligheid van de tumor van deze patiënten voor de cytostatica die gegeven worden. Verschillen in de gevoeligheid op een behandeling kunnen worden veroorzaakt door omgevingsfactoren en genetische factoren. Bij deze genetische factoren gaat het vaak om frequent aanwezige varianten in genen coderend voor enzymen, transporterts of receptoren betrokken bij farmacokinetiek, farmacodynamiek of pathogenese van de ziekte. Deze frequent voorkomende genetische varianten worden ook 'polymorfismen' genoemd. Ongeveer 3-5\% van de polymorfismen beïnvloeden het functioneren van het gen, maar deze invloed lijdt niet tot grote problemen voor de gezonde mens onder normale omstandigheden. Echter tijdens een behandeling kunnen deze polymorfismen wel van invloed op het farmacologische metabolisme van de medicamenten die gegeven worden, wat kan leiden tot meer bijwerkingen of het uitblijven van een behandelingsresultaat. Meer inzicht in de mechanismen die leiden tot ongevoeligheid voor de behandeling en de genetische factoren of genetische varianten die daarbij een rol spelen zou kunnen leiden tot een meer effectieve behandeling van patiënten met een slechte respons. Bij farmacogenetica worden de genetische varianten van genen die coderen voor enzymen, drug transporters of receptoren die betrokken zijn bij het farmacologische metabolisme bestudeerd en gebruikt om die patiënten at risk voor bijwerkingen of ongevoeligheid voor de behandeling te identificeren. In dit promotietraject was het doel het verrichten van een farmacogenetische studie om een genetisch profiel te kunnen definiëren waarmee die patiënten at risk voor een slechte response op de chemotherapie en daarmee een slechte prognose ten aanzien van survival te kunnen definiëren.

Hoofdstuk 1 omvat een algemene introductie en beschrijft de huidige behandeling van patiënten met een osteosarcoom, de relatie tussen prognostische factoren en overleving, en de huidige kennis betreffende de rol van farmacogenetica in de behandeling van patiënten met kanker. Tevens worden de doelen en inhoud van dit proefschrift beschreven.

Hoofdstuk 2 beschrijft de analyse naar de rol van leeftijd op moment van diagnose op de overlevingskans van patiënten met een osteosarcoom. In totaal werden 102 patiënten geïncludeerd voor deze studie. Voor de gehele groep was de 5 -jaars overleving 53.5\%. We vonden een significante associatie tussen leeftijd op moment van diagnose en de overlevingskans. Patiënten jonger dan 10 jaar hadden een overleving van $70.6 \%$ vergeleken met patiënten tussen 11 en 19 jaar met een overleving van $52.5 \%$ en patiënten ouder dan 20 jaar met een overleving van slechts $33.3 \%(p=0.002)$
In hoofdstuk 3 bestudeerden we of er bepaalde trends in overleving als ook in lange termijn gevolgen te identificeren zijn in kinderen met een osteosaarcoom. Hiervoor in cludeerden we 130 kinderen, welke van 1979 tot 2008 behandeld zijn voor een osteosarcoom. Deze periode van 30 jaar werd opgesplitst in 3 periodes waarin nieuwe behandelprotocollen geïmplementeerd werden (1979-1983, 1984-2002 en 2003-2008). Vergelijking van de verschillende periodes liet een verbetering van overleving van $58.6 \%$ van kinderen behandeld in de periode $1979-1983$ naar $78.6 \%$ van kinderen behandeld in de periode 2003-2008 $(p=0.13)$ zien. Het verschil in overleving kon niet verklaard worden door het gebruik van andere cytostatica dan wel andere cumulatieve doseringen. Op basis van de analyses werd dan ook geconcludeerd dat niet de chemotherapie maar de verbeterde supportive care als belangrijkste factor gezien moet worden wat heeft bijgedragen aan de verbeterde overleving . Verder laat deze studie zien dat er een evident risico is op late effecten van de behandeling zoals cardiale problemen en secundaire maligniteiten.

Hoofdstuk 4 beschrijft een methode om DNA te isoleren uit deels 30 jaar gearchiveerd formaline gefixeerd en ontkalkt weefsel. Het ontwikkelen van deze techniek was nodig gezin door de hoge mortaliteit van deze ziekte slechts van een deel van de geïncludeerde patiënten DNA aanwezig was. Echter was van alle patiënten tumorweefsel met omringend normaal weefsel in vorm van spieren of huid opgeslagen in het pathologische archief Doormiddel van deze, door ons ontwikkelde techniek, zijn wij geslaagd om betrouwbare germline DNA van het spier- of huidweefsel te isoleren.

In hoofdstuk 5 bestudeerden we in een kandidaat gen analyse genetische variaties welke invloed kunnen hebben op de eliminatie van methotrexaat (MTX). Hoog gedoseerd methotrexaat is een van de belangrijkste behandelcomponenten in de therapie van osteosarcomen. Echter ervaren patiënten veel bijwerkingen door de in de geneeskunde uitzonderlijk hoge dosering van MTX met $12 \mathrm{~g} / \mathrm{m}^{2}$. In totaal werden 640 giften van hoog gedoseerde MTX geëvalueerd. MTX-geïnduceerde toxiciteit graad 3 of 4 werd gezien in $41.8 \%$ van de patiënten. Vier polymorfismen lieten een associatie met MTX plasma spiegels zien met een p-waarde van <0.10: ABCC2 c.3972C > T, AMPD1 c.34C>T, ITPA c.94C>T en GSTT1del. Associatie analyse van deze vier polymorfismen met MTX-geïnduceerde toxiciteit liet echter geen significante associatie zien. Concluderend hebben wij aanwijzingen gevonden dat genetische variaties een invloed hebben op de hoogte van de MTX spiegel. Echter waren deze varianten niet geassocieerd met een verhoogd risico op MTX-gerelateerde bijwerkingen

In hoofdstuk 6 evalueerden we de rol van het MTHFR c.677C>T polymorfisme met betrekking tot het ontwikkelen van MTX-geïnduceerde levertoxiciteit. Hiervoor analyseerden we een cohort van 98 kinderen met een osteosarcoom dan wel acute lymfatische leukemie welke allen behandeld waren met hoog gedoseerde MTX. We vonden en 1.8 keer verhoogd risico op het ontwikkelen van levertoxiciteit bij patiënten met een T versus een $\mathrm{C}$ allel (95\% CI 1.0-3.2, $\mathrm{p}=0.04$ ). Deze bevinding werd niet bevestigd in een meta-analyse van zeven 
studies met 1044 patiënten; de OR voor 677 T versus C allel was 1.1 (95\% CI 0.84-1.5, $\mathrm{p}=0.25$ ). We concludeerden dat in patiënten met kanker, het MTHFR c.677C $>$ T polymorfisme geen duidelijke rol speelt in het ontwikkelen van MTX-geïnduceerde levertoxiciteit.

In hoofdstuk 7 evalueerden we polymorfismen in het TPMT en COMT gen welke in een onlangs gepubliceerd artikel in Nature Genetics in verbinding werden gebracht het ontwikkelen van cisplatin-geïnduceerde ototoxiciteit. Hiervoor analyseerden we twee onafhankelijke groepen van patiënten met een osteosarcoom, respectievelijk 110 en 38 patiënten en verrichtten een meta-analyse samen met alle tot nu gepubliceerde studies op dit gebied van in totaal 664 patiënten met kanker. Enkel het rs4646316 polymorfisme in het COMT gen liet een significante associatie zien met het ontwikkelen van ototoxiciteit (OR A versus T allele: 1.5, 95\% CI: 1.14-1.97, p=0.003). Het effect was echter veel kleiner dan in het gepubliceerde artikel in Nature Genetics. Hieruit concludeerden we, dat de invloed van polymorfismen in het TPMT dan wel COMT gen op het ontwikkelen van cisplatin-geïnduceerde ototoxiciteit minder beduidend is dan eerst aangenomen.

In hoofdstuk 8 werden genetische variaties van genen betrokken in de metabole pathways van cisplatin en doxorubicin bestudeerd in relatie tot de progressievrije overleving (PFS) bij patiënten met een osteosarcoom. We analyseerden 381 polymorfismen in 54 genen in 177 patiënten met gebruikmaking van een Illumina Bead Array systeem. De sterkste associaties met PFS werden gevonden voor polymorfismen in FasL, MSH2, CASP3, $A B C C 5$ en CYP3A4. Gebaseerd op de aanwezigheid van deze genetische factoren werd per patiënt een genetische score berekend. Patiënten zonder of enkel een risico allel hadden een 5 -jaars PFS van $100 \%$ vergeleken met een 5 -jaar PFS van $84.4 \%$ in patiënten met 2 of 3 risico allelen, $66.7 \%$ in patiënten met 4 of 5 risico allelen en $41.8 \%$ in dragers van meer dan 5 risico allelen. Met deze studie hebben wij genen aan kunnen geven welke mogelijk essentieel zijn voor de uitkomst in de behandeling van het osteosarcoom.

Hoofdstuk 9 geeft een algemene discussie waarin de conclusies van de verschillende studies worden samengevat en wordt ingegaan op wat dit betekent voor vervolgonderzoek bij patiënten met een osteosarcoom. 


\section{Dankwoord}

About the author

List of publications

List of abbreviations 


\section{Dankwoord}

Fertig! Wat ik geleerd heb is, dat volhardendheid en zelfdiscipline zich betaald maken, en vooraf een plan B hebben als A niet gaat lukken. Ik ben dankbaar voor deze periode - ook dat het nu klaar is - en wil graag de belangrijkste mensen om mij heen bedanken voor hun steun en het vertrouwen.

Allereerst dank ik alle patienten en hun ouders, die in de loop van de jaren hun steentje aan dit proefschrift hebben bijgedragen.

Beste Peter, je kritische vragen waren niet altijd gemakkelijk te beantwoorden maar hebben het stuk absoluut sterker uit de strijd doen komen. Ons uitje naar Amsterdam waar ik na een geslaagde presentatie voor de Kika-commissie bij jou, de professor, achter op een OV-fiets door de P.C. Hooftstraat, met 2 pakjes gratis soep in zak, richting station terug reed, levert nog steeds een leuke anekdote op voor al mijn Oostenrijkse vrienden, waar dit onmogelijk zou zijn.

Beste Henk-Jan, dank voor de samenwerking. Je snelle respons op mijn artikelen heb ik zeer gewaardeerd!

Beste Maroeska, zonder jou was ik nooit begonnen, en had ik dit ook nooit tot een eind kunnen brengen. Je bent echt een duizendpoot waar ik dagelijks met bewondering naar sta te kijken. Je enthousiasme voor het onderzoek is aanstekelijk en laat andere dingen,zo als het halen van een vlucht naar Chicago, even op de achtergrond komen te staan. Bedankt voor je vertrouwen in mij als kinderoncoloog en als promovendus op dit onderzoek.

Beste Marieke, bij jou kon ik altijd even terecht. Dankzij jou wist ik me een weg te banen door de wereld van ORs, 95\% CI, p-waarden in verhouding tot die ene SNP. Zonder jouw inzet en zorgvuldigheid had ik de analysen van dit boekje nooit kunnen uitvoeren.

Ik dank de leden van de manuscriptcommissie Prof. Kiemeney, Prof. Caron en Prof. Russel, voor de kritische maar ook snelle beoordeling van dit manuscript.

Beste mensen van het lab, door op het ene lab mijn werkplek te hebben en het andere lab het werk te doen was ik een vreemde eend in de bijt op beide afdelingen. Ik heb echter met veel plezier gewerkt bij de genetica en voelde me op mijn plek bij het lab kinderoncologie. Dank voor de openhartigheid en fijne werksfeer waarin ik me thuis heb gevoeld.
Beste co-autheurs bedankt voor de samenwerking aan de diverse projecten.

Hanneke, bedankt voor je inspanningen voor dit proefschrift en veel succes met de afronding van je eigen onderzoek.

Lieve Winterkolderaars, jullie onderschatten waarschijnlijk de impact van ,ons' kamp op dit proefschrift. De winterkolderweken waren en zijn nog steeds mijn grootste motivatie om iets te betekenen voor kinderen met een osteosarcoom. Bedankt voor alle gezelligheid, 10 over 3 momenten en op nog vele jaren Winterkolder.

Tijdens mijn tijd als eeuwige kinderarts-assistent was het een komen een gaan van mede-assistenten. In het bijzonder wil ik degenen noemen met wie ik lief en leed heb gedeeld, Judith, Joyce, Karin en Kim. Dank voor de mogelijkheid om zo nu en dan stoom af te blazen.

Jos, wat fijn dat ik mijn aios tijd onder jouw hoede heb mogen beleven. Bedankt voor de moeite die je hebt gedaan zodat ik zonder problemen mijn opleiding stop mocht zetten voor het werk op het lab.

Sjors, zonder jou was ik naar een paar maanden gillend terug gelopen naar Oostenrijk. En kijk, nu komt Nederland niet meer van mij af!

Pim, dank voor je begeleiding tijdens mijn periode in Tilburg. Het was de grondsteen voor mijn interesse in de kinderoncologie.

Beste kinderoncologen, het zijn turbulente jaren. Bedankt voor jullie steun en vertrouwen in mij voor dit vak.

Dann noch alle Freunde verstreut über den ganzen Kontinent. Auch wenn der Kontakt weniger geworden ist, die Qualität bleibt. Danke für Eure Freundschaft.

Lieve Marije, je bent de meest oprechte persoon die ik ken. Als ik een eerlijk antwoord wi hebben dan moet ik bij jou zijn. Ik ben blij dat onze vriendschap stand houdt, ondanks de drukte en de afstand, want het zou echt een gemis zijn. Bedankt dat je vandaag bij me bent. 
Lieve Stefanie, wat een geluk voor mij dat je in de zelfde periode op het lab werkte. Samen zijn we gegroeid in onze taken, samen kijken we soms met verwondering naar de Nederlanders', samen hebben we gelachen en gehuild en in een ijskoud bergmeertje gezwommen. Ik ben blij dat jij vandaag achter me staat.

Liebe Hildegard, lieber Hartwig, danke für Eure sprichwörtlich grenzenlose Unterstützung.

Liefste Martin, wat ben je aanwezig door afwezig te zijn.

Liebster Michael, durch dich bin ich wie ich bin. Ich liebe dich aus ganzem Herzen.

Liebe Mam und Dad, es war nicht immer einfach, unser Rucksack ist grösser als der Durchschnitt. Danke für Eure Liebe und Euer Verständis.

Liebster Bernhard, bedankt voor alle tijd die je me hebt laten nagelbijten op dit proefschrift, je vulde me aan en zorgde voor de nodige rust. Maar het allermooiste is dat we samen Jakob en Nora hebben, wat zijn ze prachtig. Je bent mijn grote liefde, met jou naast me kan alles en is alles goed.

\section{About the author}

Melanie Margaretha Hagleitner was born on the $12^{\text {th }}$ of October 1978 in Zell am See, Austria. From 1994 till 1998 she attended secondary school at the Höhere Bundeslehranstalt in Saalfelden, Austria. In 1998 she started with the study Medicine at the Medical University of Vienna. After obtaining her doctoral degree in 2004, she moved to the Netherlands where she started working at the Emergency Room of the Admiraal de Ruyter Hospital (Dr van Lieshout) in Goes for nine months.

Her career in pediatrics began in St Elisabeth Hospital in Tilburg (Dr van Dijken). In 2006 her recidency in pediatrics started at Radboud University Medical Center in Nijmegen (D Draaisma). During this period she started with her PhD project in 2008 and worked two years fulltime at the Laboratory of Pediatric Oncology (Prof Dr Hoogerbrugge, Dr van Leeuwen) and Laboratory of Human Genetics (Dr Coenen). Training in Pediatrics continued in Jeroen Bosch Hospital in Den Bosch (Dr Hoekstra and Dr de Vries) and Rijnstate Hospital in Arnhem (Dr van Setten and Dr Landstra).

In the period 2010-2011 she attended the TULIPS (Training Upcoming Leaders in Pediatric Science) PhD Curriculum. Furthermore, she was active member of the committee of Young Members of the Dutch Federation of Pediatrics (JA-NVK) from 2009 until 2013.

In 2014 she started her Fellowship in Pediatric Oncology at Radboud University Medical Center in Nijmegen (Dr te Loo). She was grateful to receive several prizes for her research work like the Tom Voûte Young Investigator Award 2012, the SIOP Fasanelli prize 2012 , Tulips Late Breakers Abstract 2013 and 3 $3^{\text {rd }}$ prize of NVK Young Investigator prize 2014. Melanie lives in Nijmegen with her partner Bernhard and their children Jakob and Nora. 


\section{List of Publications}

Gennery AR, Slatter MA, Bredius RG, Hagleitner MM, Weemaes C, Cant AJ, Lankester AC Hematopoietic stem cell transplantation corrects the immunologic abnormalities associated with immunodeficiency-centromeric instability-facial dysmorphism syndrome. Pediatrics. 2007:120:1341-1344.

Hagleitner MM, Lankester A, Maraschio P, Hultén M, Fryns JP, Schuetz C, Gimelli G, Davies EG, Gennery A, Belohradsky BH, de Groot R, Gerritsen EJ, Mattina T, Howard PJ, Fasth A, Reisli I, Furthner D, Slatter MA, Cant AJ, Cazzola G, van Dijken PJ, van Deuren M, de Greef JC, van der Maarel SM, Weemaes CM. Clinical spectrum of Immunodeficiency, centromeric instability and facial dysmorphism (ICFsyndrome). J Med Genet. 2008;45:93-99.

Hagleitner MM, Coenen MJH, Jeuken JWM, Flucke U, Schreuder HW, te Loo DMWM, Hoogerbrugge PM. Reliability of genotyping decalcified and paraffin embedded tissue from patients with osteosarcoma. Pediatric Blood \& Cancer. 2011;56:35-38.

Hagleitner MM, Hoogerbrugge PM, van der Graaf WT, Flucke U, Schreuder HW, Te Loo DM. Age as prognostic factor in patients with osteosarcoma. Bone. 2011;49:1173-1177.

Hagleitner MM, Mascini EM, van Berkel S, Bosman J, Mulder JC, van Setten P. Foreign Adopted Children are a Source of Methicillin-Resistant Staphylococcus aureus Transmission to Countries with Low Prevalence. Pediatr Infect Dis J. 2012;31:655-658.

Hagleitner MM, de Bont ESJM, te Loo DMWM; Survival trends and long term toxicity in pediatric patients with osteosarcoma. Sarcoma. 2012:636405.

Hagleitner MM, Coenen MJ, Aplenc R, Patiño-Garcia A, Chiusolo P, Gemmati D, De Mattei $M$, Ongaro A, Krajinovic M, Hoogerbrugge PM, Vermeulen SH, Te Loo DM. The role of the MTHFR 677 C $>$ T polymorphism in methotrexate-induced liver toxicity: a meta-analysis in patients with cancer. Pharmacogenomics J. 2014;14:115-119.

Hagleitner MM. Eerste resultaten van farmacogenetisch onderzoek bij patiënten met een osteosarcoom: op weg naar geïndividualiseerde behandeling. Kinderarts en Wetenschap. 2014;9:14-15.

te Loo DMWM, Hagleitner MM, Coenen MJH. Is there a role for the MTHFR 677C>T and the $1298 \mathrm{~A}>\mathrm{C}$ polymorphism in methotrexate induced liver toxicity? Editorial. Pharmacogenomics. 2014;15:1401-1403.
Hagleitner MM, Mascini EM, van Berkel S, Bosman J, Mulder JC, van Setten P. MRSA screening buitenlandse adoptiekinderen: de moeite waard! Infectieziekten Bulletin. 2014; 10:280-283

Hagleitner MM. Farmacogenetisch onderzoek bij patienten met een osteosarcoom. Kinderarts en Wetenschap. 2014:12:27-28.

Hagleitner MM, Coenen MJH, Patiño-Garcia A, Vos HI, Gelderblom AJ, Hoogerbrugge PM, Guchelaar H-J, te Loo DMWM. Genetic variants in the TPMT and COMT genes are not associated with cisplatin induced hearing loss in patients with cancer. PLOS One. 2014;9:e115869.

Hagleitner MM, Coenen MJH, Gelderblom AJ, Hoogerbrugge PM, Guchelaar H-J, te Loo DMWM. First steps to personalized medicine in osteosarcoma: Pharmacogenetics as predictive marker of outcome after chemotherapy based treatment. In revision, Clinical Cancer Research. 


\section{List of abbreviations}

\begin{tabular}{ll} 
6MP & 6-Mercaptopurine \\
ABCC & ATP-binding cassette, subfamiliy C, member \\
ALL & Acute lymphoblastic leukemia \\
ALT & Alanine transferase \\
AMPD & Adenosine monophosphate deaminase \\
AP & Alkaline phosphatase \\
ASCO & Amercian Society of Clinical Oncology \\
bP & Basepair \\
CASP & Caspase \\
CI & Confidence Intervall \\
COG & Children's Oncology Group \\
COMT & Catechol O-methyltransferase \\
CTCAE & Common terminology criteria for adverse events \\
CYP & P450 cytochrome \\
DFS & Disease free survival \\
DHFR & Dihydrofolatereductase \\
DMET & Drug metabolism enzymes and transporters \\
DNA & Deoxyribonucleic acid \\
DPYD & Dihydropyrimidine dehydrogenase \\
EURAMOS & The European and Amercian Osteosarcoma Study Group \\
FasL & FasLigand \\
FFPE & Formalin-fixed, paraffin-embedded \\
FS & Fractional shortening \\
GEE & Generalized estimated equations \\
GGH & Gamma-glutamyl hydrolase \\
GSTT & Glutathione S-transferase \\
GWAS & Genome wide association studies \\
HER & Human epidermal growth factor receptor \\
HR & Hazard ratio \\
HWE & Hardy-Weinberg equilibrium \\
IFNa & Interferon a \\
ITPA & Inosine triphosphate pyrophosphatase \\
LD & Linkage disequilibrium \\
LDH & Lactate dehydrogenase \\
MAF & Minor allele frequency \\
MDR & Multifactor dimensionality reduction \\
& \\
\hline
\end{tabular}

$\begin{array}{ll}\text { MSH } & \text { Mut S homologue } \\ \text { MTHFR } & \text { Methylenetetrahydrofolate reductase } \\ \text { MTP-PE } & \text { Muramyl tripeptide-phosphatidyl ethanolamine } \\ \text { MTX } & \text { Methotrexate } \\ \text { NCBI } & \text { National Center for Biotechnology Information } \\ \text { NCI } & \text { National Cancer Institute } \\ \text { OD } & \text { Optical density } \\ \text { OR } & \text { Odds Ratio } \\ \text { OS } & \text { Overall survival } \\ \text { PCR } & \text { Polymerase chain reaction } \\ \text { PFS } & \text { Progression free survival } \\ \text { RA } & \text { Rheumatoid arthritis } \\ \text { RNA } & \text { Ribonucleic acid } \\ \text { ROS } & \text { Reactive oxygen species } \\ \text { SIOP } & \text { International Society of Paediatric Oncology } \\ \text { SLC } & \text { Salute carrier } \\ \text { SMN } & \text { Secondary malignancy neoplasm } \\ \text { SNPS } & \text { Single nucleotide polymorphisms } \\ \text { TPMT } & \text { Thiopurine 5-methyltransferase } \\ \text { WBC } & \text { White blood cell }\end{array}$


wann - wenn nicht jetzt

wo - wenn nicht hier

wie - wenn ohne Liebe

wer - wenn nicht wir

Rio Reiser 
